\title{
Desenvolvimento de um Modelador de Movimentos para o Sistema Freeflow-3D
}

\author{
Luciana Santos Paiva
}

Orientador: Prof. Dr. Antonio Castelo Filho

Monografia apresentada ao Instituto de Ciências Matemáticas e de Computação - ICMC-USP, para o Exame de Qualificação, como parte dos requisitos para obtenção do título de Doutor em Ciências - Área: Ciências de Computação e Matemática Computacional.

USP - São Carlos

Agosto de 2000 
"Se você não tem a oportunidade de fazer grandes coisas, pode fazer pequenas coisas de uma forma grandiosa." Brian Weiss

"A felicidade não está em viver, mas em saber viver. Não vive mais o que mais vive, mas o que melhor vive." Mahatma Gandhi 
Dedico este trabalho aos meus pais,

Nelson e Ana,

ao meu noivo Armando Luis e ao meu avô Antonio, com saudades. 


\section{Agradecimentos}

A Deus, que me protege e ilumina o meu caminho nesta missão, que é a vida.

Ao Prof. Castelo pela orientação dedicada, pela ativa participação, pela paciência e pelos conhecimentos transmitidos durante todo o decorrer deste trabalho.

Aos meus pais, Nelson e Ana, pelo carinho, incentivo e apoio em todos os momentos da minha vida.

Ao meu noivo Armando Luis pelo amor, carinho e compreensão, que soube suportar a minha ausência e me confortar nos momentos difíceis.

À minha família pelo apoio e incentivo neste período de muitas ausências e em especial à minha avó Laudina, pelas orações.

Ao pessoal do LCAD e do grupo de Análise Numérica, pelas constantes sugestões e pela amizade. Em especial ao prof. Norberto, ao prof. Murilo, ao prof. Armando, ao prof. Gustavo e ao prof. Poti, pelos ensinamentos.

À professora Ângela, do centro cultural, pelas aulas de inglês e por nossa amizade.

Aos meus professores de graduação do departamento de matemática da UFSCar, em especial ao prof. Ivo Machado da Costa, que abriu as portas para a realização deste trabalho.

À amiga Christiane pela grande amizade desde a época da graduação.

À Juliana, Aninha e Rodrigo, pela colaboração nos estudos, pelas inúmeras dúvidas esclarecidas e principlamente pela amizade.

Aos amigos Luciane, Lilian, Sandra, Rubia, Selma, Silvio, Maristela, Ricardo, Luiz Fernando, Fabio, Maju, Dudu, Helton, Marcelo, F. Pio, Juliana M., pelos momentos de descontração e pela amizade.

Aos funcionários do ICMC, especialmente aos da pós-graduação, Beth, Laura, Marília, Adriana e Sandra, pela simpatia e atenção com que sempre me atenderam.

À agência FAPESP pelo suporte financeiro no desenvolvimento deste trabalho.

E finalizando, gostaria de agradecer a todos aqueles que de alguma maneira contribuíram para a realização deste trabalho. 


\section{Abstract}

This work presents a modeller of movements to integrate the Freeflow-2D and Freeflow$3 \mathrm{D}$ systems. These systems consist of a modelling, a simulation and a visualization enviroment for incompressible flows with free surfaces. The movement of each object used during the simulation was defined from its mass center. Three types of movement was implemented: rotation, simple harmonic and cubic splines interpolation. Simulation examples of problems with object movement are presented, confirming the ability of the movement modeller in dealing with the complexities of these simulations. 


\section{Resumo}

Este trabalho apresenta um modelador de movimentos para integrar os sistemas Freeflow2D e Freeflow-3D. Estes sistemas compreendem um ambiente de modelagem, simulação e visualização de escoamentos do tipo incompressíveis com superfícies livres. O movimento de cada objeto utilizado durante a simulação é definido a partir do seu centro de massa. Foram implementados três tipos de movimento: rotação, harmônico simples e interpolação por splines cúbica. Exemplos de simulaçōes que envolvem problemas com objetos em movimento são apresentados, confirmando o desempenho do modelador de movimentos. 


\section{Sumário}

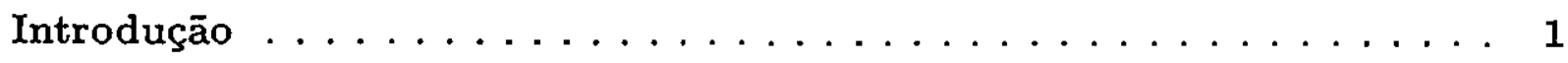

1 Equaçōes Governantes $\quad 4$

1.1 Considerações Iniciais . . . . . . . . . . . . . . . . . . . 4

1.2 Noções Fundamentais . . . . . . . . . . . . . . . . . . . 4

1.3 As Equações de Navier-Stokes . . . . . . . . . . . . . . . . . 6

1.4 Desenvolvimento das Equações . . . . . . . . . . . . . . 7

1.5 As Condições de Contorno na Superfície Livre . . . . . . . . . . . . . . 12

2 O Método GENSMAC $\quad 15$

2.1 Consideraçōes Iniciais . . . . . . . . . . . . . . . . . . . 15

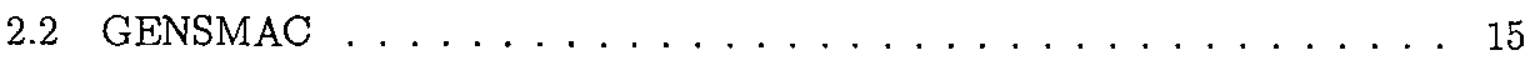

2.3 Algoritmo Computacional .................... . 16

2.4 Definição das Células . . . . . . . . . . . . . . . . . . . . . 19

2.5 Condições de Contorno na Superfície Livre . . . . . . . . . . . . . . . . . 20

3 Discretizaçōes $\quad 23$

3.1 Considerações Iniciais . . . . . . . . . . . . . . . . . . . . . . 23

3.2 Discretização do Domínio . . . . . . . . . . . . . . . 23

3.3 Discretização das Equações . . . . . . . . . . . . . . . . . . . 25

3.4 O Método das Diferenças Finitas . . . . . . . . . . . . . . . 25

3.5 A Discretização da Equação de Momento . . . . . . . . . . . . . . 26

3.6 Condições de Contorno na Superfície Livre . . . . . . . . . . . . . . . 35

3.7 Condiçōes de Contorno na Superfície Rígida . . . . . . . . . . . . . 37

3.8 A Discretização da Equação de Poisson . . . . . . . . . . . . . . . . . . 39

3.9 A Solução da Equação de Poisson . . . . . . . . . . . . . . . . . . 41 
3.10 Controle do Passo no Tempo . . . . . . . . . . . . . . . . . . 42

3.11 O Movimento das Partículas . . . . . . . . . . . . . . . . 43

4 O Ambiente Freeflow-3D $\quad 44$

4.1 Consideraçōes Iniciais . . . . . . . . . . . . . . . . . . . . . . 44

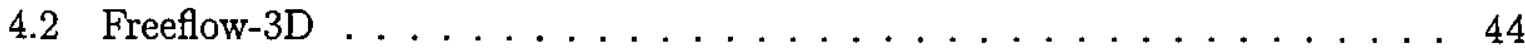

4.3 Estrutura de Dados . . . . . . . . . . . . . . . . . 45

4.4 O Modelador . . . . . . . . . . . . . . . . 47

4.5 o Simulador . . . . . . . . . . . . . . . . . . 49

4.6 O Visualizador ............................... 51

4.7 O Reiniciador . . . . . . . . . . . . . . . . . . . 52

4.8 A Comunicação Entre os Módulos . . . . . . . . . . . . . . . . . 53

5 O Modelador de Movimentos $\quad 55$

5.1 Considerações Iniciais . . . . . . . . . . . . . . . 55

5.2 O Modelador de Movimentos Bidimensional . . . . . . . . . . . . 55

5.2 .1 Determinação do Centro de Massa . . . . . . . . . . . . 56

5.2.2 Definição do Movimento de Translação e Rotação a partir do Centro

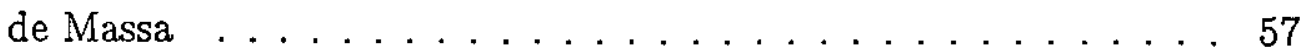

5.2 .3 Movimento de Rotação . . . . . . . . . . . . . . . 61

5.2 .4 Movimento Harmônico Simples . . . . . . . . . . . . . . . . 62

5.2.5 Movimento Definido por Interpolação por Splines Cúbica . . . . . . 64

5.3 O Modelador de Movimentos Tridimensional . . . . . . . . . . . . . 72

5.3.1 Definição do Movimento de Translação e Rotação a partir do Centro

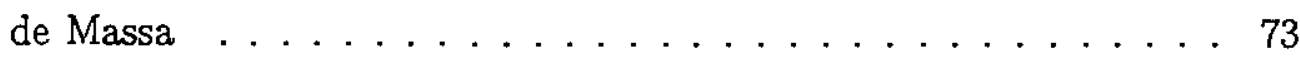

5.3 .2 Movimento de Rotação . . . . . . . . . . . . . . 78

5.3.3 Movimento Harmônico Simples . . . . . . . . . . . . . . . . 80

5.3.4 Movimento Definido por Interpolação por Splines Cúbica . . . . . . 81

6 Exemplos Numéricos $\quad 86$

6.1 Considerações Iniciais . . . . . . . . . . . . . . . . . . 86

6.2 Exemplos Bidimensionais . . . . . . . . . . . . . . 86

6.3 Exemplos Tridimensionais . . . . . . . . . . . . . 96 
7 Conclusōes

Referências Bibliográficas . . . . . . . . . . . . . . 112 


\section{Lista de Figuras}

2.1 Domínio, malha e células . . . . . . . . . . . . . . 20

2.2 Célula (S) com o lado direito em contato com uma célula (V) . . . . . 20

2.3 Célula $(\mathrm{S})$ com dois lados em contato com células $(\mathrm{V}) \ldots \ldots \ldots 21$

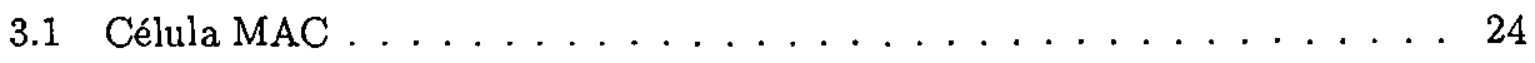

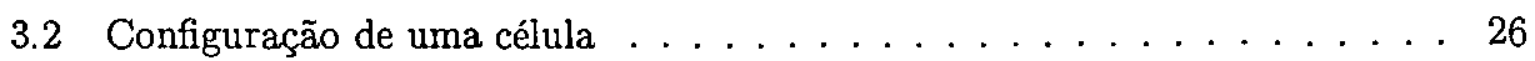

3.3 Discretização da célula (S) com o lado direito em contato com uma célula

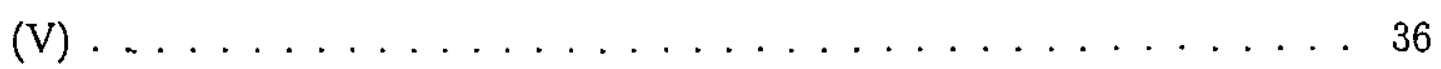

3.4 Discretização da célula (S) com dois lados em contato com células (V) . . 36

3.5 Célula $\mathrm{F}$ com a face $i+\frac{1}{2}$ em contato com células $\mathrm{S}$ ou $\mathrm{C} \ldots \ldots . \ldots 38$

3.6 Célula $\mathrm{F}$ com a face $i+\frac{1}{2}$ e $j+\frac{1}{2}$ em contato com células $\mathrm{S}$ ou $\mathrm{C} \ldots \ldots 39$

4.1 Face, células e intersecçōes. . . . . . . . . . . . . . . . . . 48

4.2 Célula, discretização da pressão e velocidade . . . . . . . . . . . . 49

4.3 Células e intersecções com as retas $\ldots \ldots \ldots \ldots \ldots \ldots$

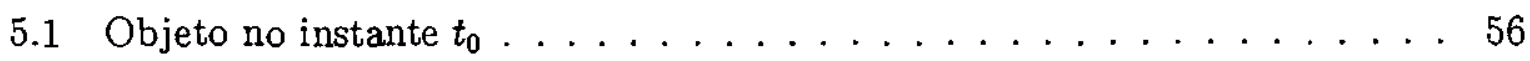

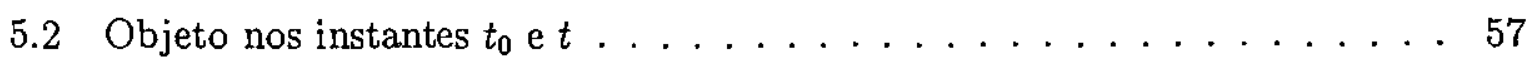

5.3 Contêiner do tipo Box inclinado . . . . . . . . . . . . . 59

5.4 Entrada de dados dos objetos . . . . . . . . . . . . . . 60

5.5 Movimento de Rotação $\ldots \ldots \ldots$. . . . . . . . . . . . 61

5.6 Movimento Harmônico Simples $\ldots \ldots \ldots 63$

5.7 Aproximação por splines . . . . . . . . . . . . . 65

5.8 Entrada dos Dados . . . . . . . . . . . . . . . . . . 65

5.9 Pontos de Interpolação . . . . . . . . . . . . . . . 66

5.10 Interpolaçāo por splines para $x_{c} \ldots \ldots \ldots \ldots 6$ 
5.11 Interpolação por splines cúbica para $\theta \ldots$. . . . . . . . . . . 67

5.12 Interpolação por splines cúbica para $x_{c} \ldots \ldots \ldots$. . . . . . . . . 69

5.13 Interpolação por splines cúbica para $y_{c} \ldots \ldots \ldots$. . . . . . . . . 70

5.14 Interpolação por splines cúbica para $\theta \ldots \ldots \ldots$. . . . . . . 71

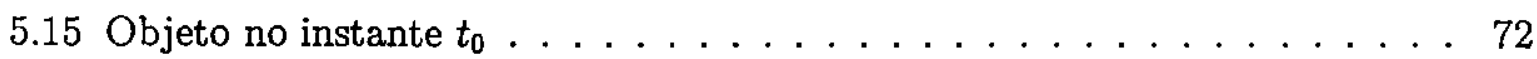

5.16 Objeto nos instantes $t_{0}$ e $t \ldots \ldots \ldots \ldots$. . . . . . . . . . . .

5.17 Contêiner do tipo Open Box . . . . . . . . . . . . . . . 76

5.18 Entrada de dados de um contêiner do tipo Open Box . . . . . . . . 76

5.19 Tipos de contêineres . . . . . . . . . . . . . . 77

5.20 Entrada de dados de um injetor do tipo Cylinder . . . . . . . . . . 77

5.21 Movimento de Rotação . . . . . . . . . . . . . . . . . . 80

5.22 Movimento Harmônico Simples . . . . . . . . . . . . . . . . . 80

5.23 Interpolação por splines cúbica para $x_{c} \ldots \ldots \ldots$. . . . . . . . . 82

5.24 Interpolação por splines cúbica para $y_{c} \ldots \ldots . \ldots . \ldots$

5.25 Interpolação por splines cúbica para $z_{c} \ldots \ldots \ldots$

5.26 Interpolação por splines cúbica para os ângulos de rotação . . . . . . . . . 85

6.1 Objeto no instante $t=0.0 \ldots \ldots \ldots 6$

6.2 Objeto no instante $t=0.0 \ldots \ldots \ldots$. . . . . . . . . . . .

6.3 Fluido no instante $t=0.006 \ldots \ldots \ldots 9$

6.4 Fluido no instante $t=0.5 \ldots \ldots \ldots \ldots$. . . . . . . . . . . . . .

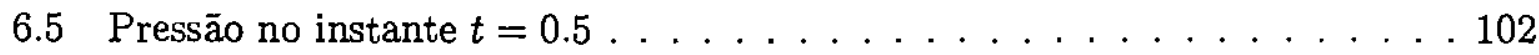

6.6 Fluido no instante $t=0.14 \ldots \ldots \ldots \ldots$

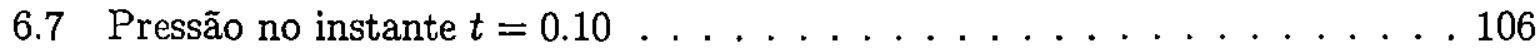

6.8 Pressão no instante $t=0.14 \ldots \ldots \ldots \ldots$

6.9 Fluido no instante $t=0.048 \ldots \ldots \ldots$. . . . . . . . . . . . . . .

6.10 Pressão no instante $t=0.048 \ldots \ldots \ldots \ldots$ 


\section{Introdução}

A dinâmica dos fluidos tem sido, nos últimos 30 anos, uma das mais interessantes fronteiras exploradas pela pesquisa e a obtenção de soluções para as equações governantes da mecânica dos fluidos representa um dos maiores desafios da computação científica.

Novas técnicas numéricas em conjunto com o contínuo avanço dos computadores digitais tornaram a simulação dos problemas em mecânica dos fluidos muito mais precisos.

A versāo moderna das indústrias alimentícias, siderúrgicas e de plásticos poderá contar, em breve, com programas para a simulação de preenchimentos de cavidades e injeção em moldes oriundos desses trabalhos.

As pesquisas que visam tratar escoamentos de fluidos com superfícies livres têm obtido sucesso utilizando-se o método de diferenças finitas. Harlow e Welch introduziram o método MAC (Marker-and-Cell) [12] usado, então, como uma das primeiras tentativas a resolver, de modo satisfatório, problemas com superfícies livres. Neste método o fluido é representado utilizando-se partículas virtuais.

Uma simplificação do método MAC foi desenvolvida por Amsden e Harlow denominado SMAC [2]. Por esse processo os ciclos de cálculo são divididos em velocidade e pressão. Isso fez com que as dificuldades encontradas no método MAC acerca da iteratividade no cálculo da velocidade e pressão não mais existissem.

Tendo como base esse novo conceito (método SMAC), Tomé e McKee desenvolveram o método GENSMAC [22], para escoamentos bidimensionais de fluidos incompressíveis com superfícies livres e domínios complexos e depois para fluidos nāoNewtonianos [23].

Existe um projeto em desenvolvimento no ICMC-USP denominado SNENS: Solução Numérica das Equaçōes de Navier-Stokes. O objetivo deste projeto é a simulação de escoamentos de fluidos e, em particular, escoamento de fluidos com superfícies livres.

$\mathrm{O}$ ambiente integrado para simulação de escoamentos incompressíveis com su- 
perfíciés livres tridimensionais desenvolvido neste projeto é denominado Freeflow-3D [3]. Este sistema é composto pelos seguintes módulos: um modelador de moldes e escoamento - Modflow-3D, um simulador de escoamentos - Simflow-3D, um visualizador de escoamentos - Visflow-3D e um reiniciador de escoamentos - Resimflow-3D. A estrutura de dados do Freeflow-3D foi planejada para permitir a manutenção e extensāo de seus módulos.

\section{Motivação e Objetivos}

Modelos de escoamento onde os objetos se movimentam são muito comuns no cotidiano. Por exemplo, na indústria alimentícia, os recipientes movimentam-se em esteiras e, na injeção de fluido, o injetor se movimenta no sentido vertical, aproximando-se do recipiente enquanto enquanto injeta o fluido.

Pensando-se no movimento de recipientes e injetores nas indústrias, este projeto de mestrado teve como objetivo desenvolver um modelador de movimentos para os objetos rígidos, utilizados na simulação de escoamentos, para ser integrado ao módulo Modflow3D. Seu desenvolvimento utilizou técnicas de aproximação e interpolação para definir a trajetória e orientação dos objetos rígidos durante a simulação.

\section{Organização da Dissertação}

Esta dissertação está organizada em sete capítulos. Os três primeiros capítulos abordam aspectos teóricos sobre escoamentos de fluidos com superfícies livres. O capítulo seguinte apresenta o sistema Freeflow-3D e os capítulos 5 e 6 descrevem o modelador de movimentos. $\mathrm{O}$ capítulo 7 apresenta as conclusões deste trabalho.

A seguir, descreve-se brevemente o conteúdo de cada capítulo:

- O Capítulo 1 apresenta um estudo das Equações de Navier-Stokes e condições de fronteira para escoamentos com superfícies livres.

- O Capítulo 2 descreve o método GENSMAC. 
- O Capítulo 3 apresenta as discretizações das equações, utilizando-se o método de diferenças finitas.

- O Capítulo 4 descreve o ambiente Freeflow-3D.

- O Capítulo 5 apresenta os processos e métodos empregados no desenvolvimento do modelador de movimentos e sua interface gráfica.

- O Capítulo 6 trata da apresentação de alguns exemplos de simulações empregando objetos em movimento.

- O Capítulo 7 finaliza esta dissertação apresentando as conclusões e contribuições deste trabalho. 


\section{Capítulo 1}

\section{Equações Governantes}

\subsection{Considerações Iniciais}

Este capítulo define os conceitos básicos da mecânica dos fluidos [9] e descreve as equações de Navier-Stokes para os escoamentos incompressíveis de fluidos com superfícies livres. Em seguida, discute o desenvolvimento dessas equações em coordenadas cartesianas e suas simplificações. Por fim, apresenta as condições de fronteira apropriadas para essas equações.

\subsection{Noções Fundamentais}

Um fluido é uma substância que se deforma continuamente quando submetida a uma tensão de cisalhamento, não importando o quanto pequena possa ser essa tensão. Uma força de cisalhamento é a componente tangencial da força que age sobre a superfície. Essa força de cisalhamento dividida pela área da superfície dá origem à tensão de cisalhamento média sobre a área. Tensão de cisalhamento num ponto é o valor limite da relação entre a força de cisalhamento e a área quando esta área tende a um ponto.

Os fluidos podem ser classificados como:

- Newtonianos: existe uma relação linear entre o valor da tensão de cisalhamento aplicada e a velocidade de deformação resultante.

-Não-Newtoniano: existe uma relação não-linear entre o valor da tensão de cisalhamento aplicada e a velocidade de deformaçāo angular.

Gases e líquidos finos tendem a ser fluidos newtonianos e o sangue, entre outro 
é um exemplo de fluido não-Newtoniano.

Escoamentos Incompressíveis são os quais as variaçōes das densidades dos fluidos são desprezíveis e quando são não desprezíveis, os escoamentos são denominados compressíveis. Os Escoamentos Transientes são aqueles cujas grandezas como velocidade e pressão variam com o tempo. Caso contrário, eles são ditos Permanentes ou Estacionários.

Viscosidade é a propriedade de um fluido responsável pela resistência ao cisalhamento. O modelo Newtoniano para as tensões viscosas estabelece que para uma dada velocidade de deformação angular de um fluido, a tensão de cisalhamento é diretamente proporcional à viscosidade.

Para os gases, a viscosidade aumenta com a temperatura mas para os líquidos, diminui.

A viscosidade $\mu$ é chamada de viscosidade absoluta ou dinâmica para se evitar cunfusão com a viscosidade cinemática $\nu$, que è a relação entre viscosidade e massa específica:

$$
\nu=\frac{\mu}{\rho}
$$

Para fins de análise é feita freqüentemente a hipótese de que um fluido é nãoviscoso. Com viscosidade zerọ a tensão de cisalhamento é sempre zero, não -importando o movimento que o fluido possa ter. Se o fluịdo é também considerado incompressível (massa específica constante em todo o domínio), ele é então chamado fluido perfeito ou ideal.

Os escoamentos de fluidos viscosos classificam-se em laminares e turbulentos, conforme a respectiva estrutura interna. No regime laminar, a estrutura do escoamento é caracterizada pelo suave movimento do fluido em lâminas ou camadas. O regime turbulento é aquele cuja estrutura é caracterizada por movimentos aleatórios de partículas fluidas adicionais ao movimento principal. Qualquer tendência para instabilidade e turbulência é amortecida por forças viscosas de cisalhamento que dificultám o movimento relativo entre camadas adjacentes do fluido. A natureza de um escoamento, isto é, se laminar ou turbulento e sua posição relativa numa escala de turbulência é indicada pelo número de Reynolds:

$$
R e=\frac{\rho U L}{\mu}=\frac{U L}{\nu}
$$


onde $L$ é um comprimento, $U$ a velocidade e $\nu$ a viscosidade cinemática.

O número de Froude:

$$
F r=\frac{U}{\sqrt{L g}},
$$

em que $g$ é a gravidade, é a razão entre forças inerciais e gravitacionais. Caracteriza os escoamentos em que a gravidade tem efeito importante, como aqueles com superfícies livres.

\subsection{As Equações de Navier-Stokes}

As equaçōes que regem os escoamentos transientes, laminares e incompressíveis são as equações de Navier-Stokes [23]:

$$
\begin{gathered}
\rho \frac{D u}{D t}=\nabla \cdot \sigma+\rho \mathrm{g} \quad \text { equação de momento }- \\
\nabla \cdot u=0 \quad \text { equação da continuidade, }
\end{gathered}
$$

onde $\rho$ é a densidade, $\boldsymbol{u}(\boldsymbol{x}, t)$ a velocidade ( $\mathrm{x}$ representa a posição no espaço e t, o tempo), $\sigma$ o tensor tensão e g a aceleração gravitacional do fluido.

Serão consideradas as seguintes equações constitutivas:

$$
\begin{gathered}
\sigma=-p I+\tau \\
\tau=2 \mu(q) \mathrm{d} \\
d=\frac{1}{2}\left[(\nabla u)+(\nabla u)^{t}\right],
\end{gathered}
$$

em que p, I, $\tau$ e d denotam a pressão, o tensor identidade, o tensor extra tensão e o tensor razão de deformação de um fluido, respectivamente.

A razão de cisalhamento local q é definida por

$$
q=\left(2 \operatorname{tr}\left(d^{2}\right)\right)^{\frac{1}{2}}
$$




\subsection{Desenvolvimento das Equações}

Considerando escoamentos bidimensionais e utilizando as coordenadas cartesianas

$O_{x y}, \operatorname{com} u(x, t)=\left(\begin{array}{c}u(x, t) \\ v(x, t)\end{array}\right)$ e $x=\left(\begin{array}{l}x \\ y\end{array}\right)$ obtém-se:

$$
\nabla u=\nabla\left[\begin{array}{c}
u \\
v
\end{array}\right]=\left[\begin{array}{ll}
\frac{\partial u}{\partial x} & \frac{\partial u}{\partial y} \\
\frac{\partial v}{\partial x} & \frac{\partial v}{\partial y}
\end{array}\right]
$$

Sabendo que

então 1.5 torna-se

$$
(\nabla u)^{t}=\left[\begin{array}{ll}
\frac{\partial u}{\partial x} & \frac{\partial v}{\partial x} \\
\frac{\partial u}{\partial y} & \frac{\partial v}{\partial y}
\end{array}\right]
$$

$$
d=\frac{1}{2}\left[\begin{array}{cc}
2 \frac{\partial u}{\partial x} & \frac{\partial u}{\partial y}+\frac{\partial v}{\partial x} \\
\frac{\partial u}{\partial y}+\frac{\partial v}{\partial x} & 2 \frac{\partial v}{\partial y}
\end{array}\right]
$$

e portanto,

$$
\tau=\mu(q)\left[\begin{array}{cc}
2 \frac{\partial u}{\partial x} & \frac{\partial u}{\partial y}+\frac{\partial v}{\partial x} \\
\frac{\partial u}{\partial y}+\frac{\partial v}{\partial x} & 2 \frac{\partial v}{\partial y}
\end{array}\right]
$$

Logo, 1.3 pode ser escrita como

$$
\sigma=\left[\begin{array}{cc}
-p & 0 \\
0 & -p
\end{array}\right]+\mu(q)\left[\begin{array}{cc}
2 \frac{\partial u}{\partial x} & \frac{\partial u}{\partial y}+\frac{\partial v}{\partial x} \\
\frac{\partial u}{\partial y}+\frac{\partial v}{\partial x} & 2 \frac{\partial v}{\partial y}
\end{array}\right]=\left[\begin{array}{cc}
-p+2 \mu(q) \frac{\partial u}{\partial x} & \mu(q)\left(\frac{\partial u}{\partial y}+\frac{\partial v}{\partial x}\right) \\
\mu(q)\left(\frac{\partial u}{\partial y}+\frac{\partial v}{\partial x}\right) & -p+2 \mu(q) \frac{\partial v}{\partial y}
\end{array}\right]
$$

e portanto:

$$
\nabla . \sigma=\left[\begin{array}{c}
\frac{-\partial p}{\partial x}+\mu(q)\left(\frac{\partial^{2} u}{\partial x^{2}}+\frac{\partial^{2} u}{\partial y^{2}}\right)+\mu(q) \frac{\partial}{\partial x}\left(\frac{\partial u}{\partial x}+\frac{\partial v}{\partial y}\right)+2 \frac{\partial \mu(q)}{\partial x} \frac{\partial u}{\partial x}+\frac{\dot{\partial \mu}(q)}{\partial y}\left(\frac{\partial u}{\partial y}+\frac{\partial v}{\partial x}\right) \\
\frac{-\partial p}{\partial y}+\mu(q)\left(\frac{\partial^{2} v}{\partial x^{2}}+\frac{\partial^{2} v}{\partial y^{2}}\right)+\mu(q) \frac{\dot{\partial}}{\partial y}\left(\frac{\partial u}{\partial x}+\frac{\partial v}{\partial y}\right)+2 \frac{\partial \mu(q)}{\partial y} \frac{\partial v}{\partial y}+\frac{\partial \mu(q)}{\partial x}\left(\frac{\partial u}{\partial y}+\frac{\partial v}{\partial x}\right)
\end{array}\right] .
$$

Considerando o operador de Laplace

$$
\nabla^{2}=\frac{\partial^{2}}{\partial x^{2}}+\frac{\partial^{2}}{\partial y^{2}}
$$


e a equação da continuidade, 1.9 obtém a seguinte forma vetorial:

$$
\nabla \cdot \sigma=-\nabla p+\mu(q) \nabla^{2} u+(\nabla u) \cdot\left[(\nabla u)+(\nabla u)^{t}\right] .
$$

Fazendo uso da identidade vetorial

$$
\nabla^{2} \boldsymbol{u}=\nabla(\nabla . u)-\nabla \wedge(\nabla \wedge u)
$$

decorre de 1.2 que:

$$
\nabla^{2} u=-\nabla \wedge(\nabla \wedge u)=\left[\begin{array}{c}
\frac{\partial}{\partial y}\left(\frac{\partial v}{\partial x}-\frac{\partial u}{\partial y}\right) \\
-\frac{\partial}{\partial x}\left(\frac{\partial v}{\partial x}-\frac{\partial u}{\partial y}\right)
\end{array}\right]
$$

A partir de 1.11 a equação 1.10 se reduz a:

$$
\nabla . \sigma=\left[\begin{array}{l}
-\frac{\partial p}{\partial x}+\mu(q) \frac{\partial}{\partial y}\left(\frac{\partial u}{\partial y}-\frac{\partial v}{\partial x}\right)+\left[2 \frac{\partial u}{\partial x} \frac{\partial \mu(q)}{\partial x}+\left(\frac{\partial u}{\partial y}+\frac{\partial v}{\partial x}\right) \frac{\partial \mu(q)}{\partial y}\right] \\
-\frac{\partial p}{\partial y}+\mu(q) \frac{\partial}{\partial x}\left(\frac{\partial u}{\partial y}-\frac{\partial v}{\partial x}\right)+\left[\left(\frac{\partial u}{\partial y}+\frac{\partial v}{\partial x}\right) \frac{\partial \mu(q)}{\partial x}+2 \frac{\partial v}{\partial y} \frac{\partial \mu(q)}{\partial y}\right]
\end{array}\right]
$$

Aplicando

$$
\frac{D}{D t}=\frac{\partial}{\partial t}+(u \cdot \nabla)
$$

sobre o vetor $\mathbf{u}$, tem-se

$$
\frac{D u}{D t}=\frac{\partial u}{\partial t}+(u . \nabla) u
$$

que na forma conservativa é dado por

$$
\frac{D u}{D t}=\frac{\partial u}{\partial t}+\nabla \boldsymbol{u} \cdot \boldsymbol{u}^{t}
$$

onde:

$$
\nabla .\left(u u^{t}\right)=\nabla \cdot\left[\begin{array}{cc}
u^{2} & (u v) \\
(v u) & v^{2}
\end{array}\right]=\left[\begin{array}{c}
\frac{\partial u^{2}}{\partial x}+\frac{\partial(v u)}{\partial y} \\
\frac{\partial(u v)}{\partial x}+\frac{\partial v^{2}}{\partial y}
\end{array}\right]
$$

$\mathrm{E}$ assim, 1.32 fica:

$$
\frac{D u}{D t}=\frac{\partial u}{\partial t}+\nabla \cdot\left(u u^{t}\right)=\left[\begin{array}{l}
\frac{\partial u}{\partial t}+\frac{\partial u^{2}}{\partial x}+\frac{\partial(v u)}{\partial y} \\
\frac{\partial v}{\partial t}+\frac{\partial(u v)}{\partial x}+\frac{\partial v^{2}}{\partial y}
\end{array}\right]
$$


Dividindo 1.1 por $\rho$, tem-se:

$$
\frac{D u}{D t}=\frac{1}{\rho} \nabla \cdot \sigma+g .
$$

A partir das equações 1.12, 1.15 e 1.16 obtém-se

$$
\begin{gathered}
\frac{\partial u}{\partial t}=-\frac{1}{\rho} \frac{\partial p}{\partial x}-\frac{\partial u^{2}}{\partial x}-\frac{\partial(u v)}{\partial y}+\nu(q) \frac{\partial}{\partial y}\left(\frac{\partial u}{\partial y}-\frac{\partial v}{\partial x}\right)+\frac{1}{\rho}\left[2 \frac{\partial u}{\partial x} \frac{\partial \mu(q)}{\partial x}+\right. \\
\left.\left(\frac{\partial u}{\partial y}+\frac{\partial v}{\partial x}\right) \frac{\partial \mu(q)}{\partial y}\right]+g_{x} \\
\frac{\partial v}{\partial t}=-\frac{1}{\rho} \frac{\partial p}{\partial y}-\frac{\partial v^{2}}{\partial y}-\frac{\partial(u v)}{\partial x}-\nu(q) \frac{\partial}{\partial x}\left(\frac{\partial u}{\partial y}-\frac{\partial v}{\partial x}\right)+\frac{1}{\rho}\left[2 \frac{\partial v}{\partial y} \frac{\partial \mu(q)}{\partial y}+\right. \\
\left.\left(\frac{\partial u}{\partial y}+\frac{\partial v}{\partial x}\right) \frac{\partial \mu(q)}{\partial x}\right]+g_{y},
\end{gathered}
$$

em que $\mathrm{g}=\left(g_{x}, g_{y}\right)^{t}$.

Considerando-se que o escoamento é caracterizado por uma dimensão L, uma velocidade U e uma viscosidade cinemática $\nu$, define-se as variáveis adimensionais pelas transformações [6]:

$$
\begin{aligned}
& \mathrm{u}=U \overline{\mathrm{u}} \\
& x=L \bar{x} \\
& \nu=\nu_{0} \bar{\nu} \\
& t=\frac{L}{U} \bar{t} \\
& p=\rho U^{2} \bar{p} \\
& g=g \bar{g} .
\end{aligned}
$$

Substituindo essas transformaçōes nas equaçōes 1.17 e 1.18, obtém-se:

$$
\begin{gathered}
\frac{\partial U \bar{u}}{\partial \frac{L}{U} \bar{t}}=-\frac{1}{\rho} \frac{\partial \rho U^{2} \bar{p}}{\partial L \bar{x}}-\frac{\partial U^{2} \overline{u^{2}}}{\partial L \bar{x}}-\frac{\partial U^{2}(\overline{v u})}{\partial L \bar{y}}+\nu_{0} \bar{\nu}(q) \frac{\partial}{\partial L \bar{y}}\left(\frac{\partial U \bar{u}}{\partial L \bar{y}}-\frac{\partial U \bar{v}}{\partial L \bar{x}}\right)+ \\
\frac{1}{\rho}\left[2 \frac{\partial U \bar{u}}{\partial L \bar{x}} \frac{\partial \mu(q)}{\partial L \bar{x}}+\left(\frac{\partial U \bar{u}}{\partial L \bar{y}}+\frac{\partial U \bar{v}}{\partial L \bar{x}}\right) \frac{\partial \mu(q)}{\partial L \bar{y}}\right]+g \bar{g}_{x}
\end{gathered}
$$




$$
\begin{gathered}
\frac{\partial U \bar{v}}{\partial \bar{U} \bar{t}}=-\frac{1}{\rho} \frac{\partial \rho U^{2} \bar{p}}{\partial L \bar{y}}-\frac{\partial U^{2} \bar{v}^{2}}{\partial L \bar{y}}-\frac{\partial U^{2}(\overline{u v})}{\partial L \bar{x}}-\nu_{0} \bar{\nu}(q) \frac{\partial}{\partial L \bar{x}}\left(\frac{\partial U \bar{u}}{\partial L \bar{y}}-\frac{\partial U \bar{v}}{\partial L \bar{x}}\right)+ \\
\frac{1}{\rho}\left[2 \frac{\partial U \bar{v}}{\partial L \bar{y}} \frac{\partial \mu(q)}{\partial L \bar{y}}+\left(\frac{\partial U \bar{u}}{\partial L \bar{y}}+\frac{\partial U \bar{v}}{\partial L \bar{x}}\right) \frac{\partial \mu(q)}{\partial L \bar{x}}+\right]+g \bar{g}_{y}
\end{gathered}
$$

ou

$$
\begin{gathered}
\frac{U^{2}}{L} \frac{\partial \bar{u}}{\partial \bar{t}}=-\frac{U^{2}}{L} \frac{\partial \bar{p}}{\partial \bar{x}}-\frac{U^{2}}{L} \frac{\partial \overline{u^{2}}}{\partial \bar{x}}-\frac{U^{2}}{L} \frac{\partial(\overline{v u})}{\partial \bar{y}}+\nu_{0} \bar{\nu}(q) \frac{U}{L^{2}} \frac{\partial}{\partial \bar{y}}\left(\frac{\partial \bar{u}}{\partial \bar{y}}-\frac{\partial \bar{v}}{\partial \bar{x}}\right)+ \\
\nu_{0} \frac{U}{L^{2}}\left[2 \frac{\partial \bar{u}}{\partial \bar{x}} \frac{\partial \bar{\nu}(q)}{\partial \bar{x}}+\left(\frac{\partial \bar{u}}{\partial \bar{y}}+\frac{\partial \bar{v}}{\partial \bar{x}}\right) \frac{\partial \bar{\nu}(q)}{\partial \bar{y}}\right]+g \bar{g}_{x} \\
\frac{U^{2}}{L} \frac{\partial \bar{v}}{\partial \bar{t}}=-\frac{U^{2}}{L} \frac{\partial \bar{p}}{\partial \bar{y}}-\frac{U^{2}}{L} \frac{\partial \bar{v}^{2}}{\partial \bar{y}}-\frac{U^{2}}{L} \frac{\partial(\overline{u v})}{\partial \bar{x}}-\nu_{0} \bar{\nu}(q) \frac{U}{L^{2}} \frac{\partial}{\partial \bar{x}}\left(\frac{\partial \bar{u}}{\partial \bar{y}}-\frac{\partial \bar{v}}{\partial \bar{x}}\right)+ \\
\nu_{0} \frac{U}{L^{2}}\left[2 \frac{\partial \bar{v}}{\partial \bar{y}} \frac{\partial \bar{\nu}(q)}{\partial \bar{y}}+\left(\frac{\partial \bar{u}}{\partial \bar{y}}+\frac{\partial \bar{v}}{\partial \bar{x}}\right) \frac{\partial \bar{\nu}(q)}{\partial \bar{y}}\right]+g \overline{g_{y}} .
\end{gathered}
$$

-... Dividindo as equações 1.19 e 1.20 por $\frac{U^{2}}{L}$ :

$$
\begin{gathered}
\frac{\partial \bar{u}}{\partial \bar{t}}=-\frac{\partial \bar{p}}{\partial \bar{x}}-\frac{\partial \bar{u}^{2}}{\partial \bar{x}}-\frac{\partial(\overline{v u})}{\partial \bar{y}}+\frac{\nu_{0} \bar{\nu}(q)}{U L} \frac{\partial}{\partial \bar{y}}\left(\frac{\partial \bar{u}}{\partial \bar{y}}-\frac{\partial \bar{v}}{\partial \bar{x}}\right)+ \\
\frac{\nu_{0}}{U L}\left[2 \frac{\partial \bar{u}}{\partial \bar{x}} \frac{\partial \bar{\nu}(q)}{\partial \bar{x}}+\left(\frac{\partial \bar{u}}{\partial \bar{y}}+\frac{\partial \bar{v}}{\partial \bar{x}}\right) \frac{\partial \bar{\nu}(q)}{\partial \bar{y}}\right]+\frac{L g}{U^{2}} \overline{g_{x}} \\
\frac{\partial \bar{v}}{\partial \bar{t}}=-\frac{\partial \bar{p}}{\partial \bar{y}}-\frac{\partial \overline{v^{2}}}{\partial \bar{y}}-\frac{\partial(\overline{u v})}{\partial \bar{x}}+\frac{\nu_{0} \bar{\nu}(q)}{U L} \frac{\partial}{\partial \bar{x}}\left(\frac{\partial \bar{u}}{\partial \bar{y}}-\frac{\partial \bar{v}}{\partial \bar{x}}\right)+ \\
\frac{\nu_{0}}{U L}\left[2 \frac{\partial \bar{v}}{\partial \bar{y}} \frac{\partial \bar{\nu}(q)}{\partial \bar{y}}+\left(\frac{\partial \bar{u}}{\partial \bar{y}}+\frac{\partial \bar{v}}{\partial \bar{x}}\right) \frac{\partial \bar{\nu}(q)}{\partial \bar{y}}\right]+\frac{L g}{U^{2}} \bar{g}_{y} .
\end{gathered}
$$


Para tornar a notação menos carregada, elimina-se a barra das variáveis $\mathrm{u}, \mathrm{x}, \nu$, $y, v, t, p$ e g:

$$
\begin{gathered}
\frac{\partial u}{\partial t}=-\frac{\partial p}{\partial x}-\frac{\partial u^{2}}{\partial x}-\frac{\partial(v u)}{\partial y}+\frac{\nu_{0} \nu(q)}{U L} \frac{\partial}{\partial y}\left(\frac{\partial u}{\partial y}-\frac{\partial v}{\partial x}\right)+ \\
\frac{\nu_{0}}{U L}\left[2 \frac{\partial u}{\partial x} \frac{\partial \nu(q)}{\partial x}+\left(\frac{\partial u}{\partial y}+\frac{\partial v}{\partial x}\right) \frac{\partial \nu(q)}{\partial y}\right]+\frac{L g}{U^{2}} g_{x} \\
\frac{\partial v}{\partial t}=-\frac{\partial p}{\partial y}-\frac{\partial v^{2}}{\partial y}-\frac{\partial(u v)}{\partial x}-\frac{\nu_{0} \nu(q)}{U L} \frac{\partial}{\partial x}\left(\frac{\partial u}{\partial y}-\frac{\partial v}{\partial x}\right)+ \\
\frac{\nu_{0}}{U L}\left[2 \frac{\partial v}{\partial y} \frac{\partial \nu(q)}{\partial y}+\left(\frac{\partial u}{\partial y}+\frac{\partial v}{\partial x}\right) \frac{\partial \nu(q)}{\partial y}\right]+\frac{L g}{U^{2}} g_{y} .
\end{gathered}
$$

Assim escreve-se as equaçōes 1.17 e 1.18 na forma adimensionalizada:

$$
\begin{gathered}
\frac{\partial u}{\partial t}=-\frac{\partial p}{\partial x}-\frac{\partial u^{2}}{\partial x}-\frac{\partial(u v)}{\partial y}+\frac{1}{R e} \nu(q) \frac{\partial}{\partial y}\left(\frac{\partial u}{\partial y}-\frac{\partial v}{\partial x}\right)+ \\
\frac{1}{R e}\left[2 \frac{\partial u}{\partial x} \frac{\partial \nu(q)}{\partial x}+\left(\frac{\partial u}{\partial y}+\frac{\partial v}{\partial x}\right) \frac{\partial \nu(q)}{\partial y}\right]+\frac{1}{F r^{2}} g_{x} \\
\frac{\partial v}{\partial t}=-\frac{\partial p}{\partial y}-\frac{\partial v^{2}}{\partial y}-\frac{\partial(u v)}{\partial x}-\frac{1}{R e} \nu(q) \frac{\partial}{\partial x}\left(\frac{\partial u}{\partial y}-\frac{\partial v}{\partial x}\right)+ \\
\frac{1}{R e}\left[2 \frac{\partial v}{\partial y} \frac{\partial \nu(q)}{\partial y}+\left(\frac{\partial u}{\partial y}+\frac{\partial v}{\partial x}\right) \frac{\partial \nu(q)}{\partial x}\right]+\frac{1}{F r^{2}} g_{y} .
\end{gathered}
$$

A equação da continuidade, na forma adimensionalizada, torna-se:

$$
\frac{\partial u}{\partial x}+\frac{\partial v}{\partial y}=0 .
$$

Uma forma mais apropriada para a equação de momento e a da continuidade será

$$
\begin{gathered}
\frac{\partial u}{\partial t}=-\nabla p+N(u) \\
\nabla \cdot u=0,
\end{gathered}
$$

onde $\mathrm{N}(\mathrm{u})$ é definido como: 


$$
\begin{gathered}
N_{1}=-\frac{\partial u^{2}}{\partial x}-\frac{\partial(u v)}{\partial y}+\frac{1}{R e} \nu(q) \frac{\partial}{\partial y}\left(\frac{\partial u}{\partial y}-\frac{\partial v}{\partial x}\right)+\frac{1}{R e}\left[2 \frac{\partial u}{\partial x} \frac{\partial \nu(q)}{\partial x}+\right. \\
\left.\left(\frac{\partial u}{\partial y}+\frac{\partial v}{\partial x}\right) \frac{\partial \nu(q)}{\partial y}\right]+\frac{1}{F r^{2}} g_{x} \\
N_{2}=-\frac{\partial v^{2}}{\partial y}-\frac{\partial(u v)}{\partial x}-\frac{1}{R e} \nu(q) \frac{\partial}{\partial x}\left(\frac{\partial u}{\partial y}-\frac{\partial v}{\partial x}\right)+\frac{1}{R e}\left[2 \frac{\partial v}{\partial y} \frac{\partial \nu(q)}{\partial y}+\right. \\
\left.\left(\frac{\partial u}{\partial y}+\frac{\partial v}{\partial x}\right) \frac{\partial \nu(q)}{\partial x}\right]+\frac{1}{F r^{2}} g_{y} .
\end{gathered}
$$

A equação da razão de cisalhamento desenvolvida em coordenadas cartesianas, em que

$$
d=\frac{1}{2}\left[(\nabla u)+(\nabla u)^{\bar{t}}\right]
$$

$\mathrm{e}$

$$
d^{2}=\frac{1}{4}\left[\begin{array}{cc}
4\left(\frac{\partial u}{\partial x}\right)^{2}+\left(\frac{\partial u}{\partial y}+\frac{\partial v}{\partial x}\right)^{2} & 2 \frac{\partial u}{\partial x}\left(\frac{\partial u}{\partial y}+\frac{\partial v}{\partial x}\right)+2 \frac{\partial v}{\partial y}\left(\frac{\partial u}{\partial y}+\frac{\partial v}{\partial x}\right) \\
2 \frac{\partial u}{\partial x}\left(\frac{\partial u}{\partial y}+\frac{\partial v}{\partial x}\right)+2 \frac{\partial v}{\partial y}\left(\frac{\partial u}{\partial y}+\frac{\partial v}{\partial x}\right) & \left(\frac{\partial u}{\partial y}+\frac{\partial v}{\partial x}\right)^{2}+4\left(\frac{\partial v}{\partial y}\right)^{2}
\end{array}\right]
$$

pode ser escrita da forma:

$$
q=\left[2\left(\frac{\partial u}{\partial x}\right)^{2}+2\left(\frac{\partial v}{\partial y}\right)^{2}+\left(\frac{\partial u}{\partial y}+\frac{\partial v}{\partial x}\right)^{2}\right]^{\frac{1}{2}}
$$

\subsection{As Condições de Contorno na Superfície Livre}

Numa superfície livre, as condições de fronteira exigem que ambas as tensões normal e tangencial se anulem. Considerando uma superfície bidimensional $\operatorname{com} n=\left(n_{x}, n_{y}\right)$, um vetor normal unitário externo, e $m=\left(m_{x}, m_{y}\right)=\left(-n_{y}, n_{x}\right)$, um vetor tangente unitário, então as condições de fronteira requerem:

$$
n(\sigma \cdot n)=0
$$

$\mathrm{e}$

$$
m(\sigma \cdot m)=0
$$


De 1.8 tem-se:

$$
\sigma . n=\sigma .\left[\begin{array}{l}
n_{x} \\
n_{y}
\end{array}\right]=\left[\begin{array}{c}
\left(-p+2 \mu(q) \frac{\partial u}{\partial x}\right) n_{x}+\mu(q)\left(\frac{\partial u}{\partial y}+\frac{\partial v}{\partial x}\right) n_{y} \\
\mu(q)\left(\frac{\partial u}{\partial y}+\frac{\partial v}{\partial x}\right) n_{x}+\left(-p+2 \mu(q) \frac{\partial v}{\partial y}\right) n_{y}
\end{array}\right]
$$

Calculando $n^{t}(\sigma \cdot n)=0$, obtém-se:

$$
\begin{gathered}
\left(-p+2 \mu(q) \frac{\partial u}{\partial x}\right) n_{x}^{2}+\mu(q)\left(\frac{\partial u}{\partial y}+\frac{\partial v}{\partial x}\right) n_{x} n_{y}+\mu(q)\left(\frac{\partial u}{\partial y}+\frac{\partial v}{\partial x}\right) n_{x} n_{y}+ \\
\left(-p+2 \mu(q) \frac{\partial v}{\partial y}\right) n_{y}^{2}=0 \\
-p n_{x}^{2}+2 \mu(q)\left[\frac{\partial u}{\partial x} n_{x}^{2}+\left(\frac{\partial u}{\partial y}+\frac{\partial v}{\partial x}\right) n_{x} n_{y}+\frac{\partial v}{\partial y} n_{y}^{2}\right]-p n_{y}^{2}=0
\end{gathered}
$$

Então, a equação 1.31 pode ser escrita como:

$$
-p\left(n_{x}^{2}+n_{y}^{2}\right)+2 \rho \nu(q)\left[\frac{\partial u}{\partial x} n_{x}^{2}+\left(\frac{\partial u}{\partial y}+\frac{\partial v}{\partial x}\right) n_{x} n_{y}+\frac{\partial v}{\partial y} n_{y}^{2}\right]=0
$$

Calculando $m^{t}(\sigma \cdot n)=0$, obtém-se:

$$
\begin{gathered}
-\left(-p+2 \mu(q) \frac{\partial u}{\partial x}\right) n_{x} n_{y}-\mu(q)\left(\frac{\partial u}{\partial y}+\frac{\partial v}{\partial x}\right) n_{y}^{2}+\mu(q)\left(\frac{\partial u}{\partial y}+\frac{\partial v}{\partial x}\right) n_{x}^{2}+ \\
\left(-p+2 \mu(q) \frac{\partial v}{\partial y}\right) n_{x} n_{y}=0 \\
\vdots \\
p n_{x} n_{y}-2 \mu(q) \frac{\partial u}{\partial x} n_{x} n_{y}+\mu(q)\left(\frac{\partial u}{\partial y}+\frac{\partial v}{\partial x}\right)\left(n_{x}^{2}-n_{y}^{2}\right)-p n_{x} n_{y}+2 \mu(q) \frac{\partial v}{\partial y} n_{x} n_{y}=0
\end{gathered}
$$

E portanto, a equação 1.32 pode ser escrita como:

$$
-2 \frac{\partial u}{\partial x} n_{x} n_{y}+\left(\frac{\partial u}{\partial y}+\frac{\partial v}{\partial x}\right)\left(n_{x}^{2}-n_{y}^{2}\right)+2 \frac{\partial v}{\partial y} n_{x} n_{y}=0
$$


Introduzindo a adimensionalização, tem-se:

$$
\begin{gathered}
-\rho U^{2} \bar{p}\left(n_{x}^{2}+n_{y}^{2}\right)+2 \rho \nu_{0} \bar{\nu}(q)\left(\frac{\partial U \bar{u}}{\partial L \bar{x}} n_{x}^{2}+\left(\frac{\partial U \bar{u}}{\partial L \bar{y}}+\frac{\partial U \bar{v}}{\partial L \bar{x}}\right) n_{x} n_{y}+\frac{\partial U \bar{v}}{\partial L \bar{y}} n_{y}^{2}\right)=0 \\
-2 \frac{\partial U \bar{u}}{\partial L \bar{x}} n_{x} n_{y}+\left(\frac{\partial U \bar{u}}{\partial L \bar{y}}+\frac{\partial U \bar{v}}{\partial L \bar{x}}\right)\left(n_{x}^{2}-n_{y}^{2}\right)+2 \frac{\partial U \bar{v}}{\partial L \bar{y}} n_{x} n_{y}=0
\end{gathered}
$$

Dividindo a equação 1.35 por $\rho U^{2}$ e a equação 1.36 por $\frac{U}{L}$, obtém-se:

$$
\begin{gathered}
-\bar{p}\left(n_{x}^{2}+n_{y}^{2}\right)+\frac{2 \nu_{0} \bar{\nu}(q)}{L U}\left(\frac{\partial \bar{u}}{\partial \bar{x}} n_{x}^{2}+\left(\frac{\partial \bar{u}}{\partial \bar{y}}+\frac{\partial \bar{v}}{\partial \bar{x}}\right) n_{x} n_{y}+\frac{\partial \bar{v}}{\partial \bar{y}} n_{y}^{2}\right)=0 \\
-2 \frac{\partial \bar{u}}{\partial \bar{x}} n_{x} n_{y}+\left(\frac{\partial \bar{u}}{\partial \bar{y}}+\frac{\partial \bar{v}}{\partial \bar{x}}\right)\left(n_{x}^{2}-n_{y}^{2}\right)+2 \frac{\partial \bar{v}}{\partial \bar{y}} n_{x} n_{y}=0
\end{gathered}
$$

Simplificando a notação e utilizando o número de Reynolds na equação 1.37 , as equações 1.31 e 1.32 tornam-se:

$$
\begin{aligned}
& -p+\frac{2 \nu(q)}{R e}\left(\frac{\partial u}{\partial x} n_{x}^{2}+\left(\frac{\partial u}{\partial y}+\frac{\partial v}{\partial x}\right) n_{x} n_{y}+\frac{\partial v}{\partial y} n_{y}^{2}\right)=0 \\
& -2 \frac{\partial u}{\partial x} n_{x} n_{y}+\left(\frac{\partial u}{\partial y}+\frac{\partial v}{\partial x}\right)\left(n_{x}^{2}-n_{y}^{2}\right)+2 \frac{\partial v}{\partial y} n_{x} n_{y}=0 .
\end{aligned}
$$




\section{Capítulo 2}

\section{O Método GENSMAC}

\subsection{Considerações Iniciais}

Apresenta-se a seguir o método GENSMAC cuja utilizaçạo no estudo de escoamentos bidimensionais de fluidos incompressíveis em superfícies livres mostra-se de vital importância. Esse método consiste numa técnica de diferenças finitas aplicada sobre uma malha diferenciada e utiliza partículas marcadoras para a visualização do escoamento e localização da superfície livre do fluido.

\subsection{GENSMAC}

O método MAC (Marker-and-Cell), desenvolvido por Harlow e Welch, é uma técnica de solução para escoamentos de fluidos viscosos e incompressíveis em superfície livres baseada em diferenças finitas sobre uma malha diferenciada. Este método é caracterizado pelo uso de partículas virtuais que representam o fluido. As coordenadas destas partículas são armazenadas e se movem de uma célula a outra. Assim, estas partículas podem definir a posição da superfície livre de modo que as condições de contorno possam ser impostas. Entretanto, ao trabalhar com a equação de Poisson e com as condições de fronteira, o método se mostrou ineficiente por existir um processo iterativo envolvendo velocidade e pressão. Então, Amsden e Harlow desenvolveram o método SMAC (Simplified Marker-and-Cell), o qual evitava algumas dificuldades por dividir o ciclo de cálculo em duas partes: uma para o velocidade e outra para a pressão. Dessa forma, a equação de Poisson torna-se mais simples e requer apenas condições de fronteira homogêneas. 
O método GENSMAC (Generalized-Simplified-Marker-and-Cell), desenvolvido por Tomé e Mckee, deriva de atualizações do método SMAC para escoamentos em superfícies livres em domínios arbitrários quando a condição de não escorregamento (no-slip) e de escorregamento livre (free-slip) é imposta em fronteiras curvas.

\subsection{Algoritmo Computacional}

A idéia básica do método GENSMAC [22] é resolver as equações de Navier-Stokes sobre uma malha diferenciada no tempo $t$ e move-se as partículas virtuais resolvendo $d x / d t=u$ para calcular o valor das novas velocidades.

Suponha-se que em um dado tempo $t_{0}$ o campo de velocidades $u\left(x, t_{0}\right)$ é conhecído $\mathrm{e}$ as condiçōes de fronteira para a velocidade e pressão sejam dadas. $O$ campo de velocidades atualizado $u(x, t)$, onde $t=t_{0}+\delta t$, é calculado como segue [23]:

(i) Através de $u\left(x, t_{0}\right)$, calcula-se $q\left(x, t_{0}\right)$ e $\nu\left(q\left(x, t_{0}\right)\right)$.

(ii) Seja $\tilde{p}\left(x, t_{0}\right)$ um campo de pressão que satisfaça a condição de tensão normal correta na superfície livre em $t=t_{0}$.

(iii) Calcula-se um campo de velocidade intermediário, $\tilde{u}(x, t)$, de

$$
\frac{\partial \tilde{u}}{\partial t}=-\nabla \tilde{p}+N(u)
$$

com $\tilde{u}\left(x, t_{0}\right)=u\left(x, t_{0}\right)$, utilizando a correta condição de fronteira para $u\left(x, t_{0}\right)$.

A equação 2.1 é resolvida através do método de Euler explícito.

Subtraindo a equação 2.1 de

$$
\frac{\partial u}{\partial t}=-\nabla p+N(u)
$$

tem-se:

$$
\frac{\partial}{\partial t}(u-\tilde{u})=-\nabla(p-\tilde{p}) .
$$

Aplicando o produto vetorial $\nabla \wedge$ a ambos os lados de 2.2 tem-se:

$$
\nabla \wedge\left[\frac{\partial}{\partial t}(u-\tilde{u})\right]=\nabla \wedge(-\nabla(p-\tilde{p}))
$$

onde

$$
\nabla \cdot \wedge\left[\frac{\partial}{\partial t}(u-\tilde{u})\right]=0
$$


Logo,

$$
\frac{\partial}{\partial t}[\nabla \wedge(u-\bar{u})]=0
$$

implicando em

$$
\nabla \wedge(u-\bar{u})=f(x), \text { para alguma } f(x), t \in\left[t_{0}, t_{0}+\delta t\right] .
$$

Mas $u=\tilde{u}$ em $t=t_{0}$, então

$$
\nabla \wedge u=\nabla \wedge \tilde{u} \quad \text { em } \quad t=t_{0} \Rightarrow f(x)=0 .
$$

Com isso, segue que

$$
\nabla \wedge(u-\tilde{u})=0,
$$

para $\forall t \in\left[t_{0}, t_{0}+\delta t\right]$. Assim, as "vorticidades" associadas com $u$ e $\bar{u}$ são iguais. Entretanto, $\tilde{u}$ não satisfaz $\nabla \cdot \tilde{u}=0$. Logo, existe uma função escalar $\psi(x, t)$ tal que

$$
u(x, t)-\tilde{u}(x, t)=-\nabla \psi(x, t)
$$

e obtém-se o seguinte campo de velocidades.

Aplicando o divergente em ambos os lados, tem-se

$$
\nabla \cdot(u(x, t)-\tilde{u}(x, t))=\nabla \cdot(-\nabla \psi(x, t))
$$

e de 1.27 obtém-se a equação de Poisson para a função $\psi$ :

$$
\nabla^{2} \psi(x, t)=\nabla . \tilde{u}(x, t)
$$

(iv) As condições de fronteira para $\psi$ são:

- Condição homogênea de Newmann

$$
\frac{\partial \psi}{\partial n}=0, \quad \text { sobre a fronteira rígida; }
$$

- Condição homogênea de Dirichlet

$$
\psi=0, \quad \text { sobre a superfície livre }
$$


onde $\mathbf{n}$ é a direção normal ao contorno rígido.

De 2.3 obtém-se o campo de velocidade atualizado:

$$
u(x, t)=\tilde{u}(x, t)-\nabla \psi(x, t)
$$

(v) Cálculo da pressão.

Substituindo 2.3 em 2.2 , obtém-se

$$
\frac{\partial}{\partial t}(-\nabla \psi(x, t))=-\nabla(p-\tilde{p})
$$

comutando os operadores

$$
-\nabla\left(\frac{\partial \psi(x, t)}{\partial t}\right)=-\nabla(p-\tilde{p})
$$

e assim

$$
\frac{\partial}{\partial t} \psi(x, t)=p-\tilde{p}
$$

que pode ser aproximada por

$$
\frac{\psi(x, t)-\psi\left(x, t_{0}\right)}{\delta t} \cong p-\tilde{p}
$$

Mas para $t=t_{0} \Rightarrow u\left(x, t_{0}\right)=\tilde{u}\left(x, t_{0}\right)$, donde obtém-se:

$$
\nabla \psi\left(x, t_{0}\right)=0
$$

Portanto, $\psi\left(x, t_{0}\right)$ é constante.

Logo,

$$
p \cong \tilde{p}+\frac{\psi}{\delta t}
$$

onde $\delta t$ é o passo no tempo. Assim, o método resolve de forma explícita a equação de momento e a equação de Poisson discreta (um sistema linear simétrico e esparso para a obtenção da velocidade potencial $\psi$ ).

(vi) Atualização das posições das partículas.

O último passo trata o movimento das partículas marcadoras para sua nova posição.

Para a representação do fluido, essas partículas são criadas nos injetores e injetadas em todo o domínio, permitindo assim uma visualização do escoamento e obtenção da orientação da superfície livre. 
As coordenadas das partículas virtuais são armazenadas a cada ciclo de cálculo e então atualizadas, resolvendo-se

$$
\frac{d x}{d t}=u \quad \text { e } \quad \frac{d y}{d t}=v
$$

pelo método de Euler. Dessa forma, obtém-se as novas coordenadas da partícula, que determina se uma partícula se move para dentro de uma nova célula ou deixa a região de domínio do fluido através de um ejetor.

\subsection{Definição das Células}

Como o fluido está continuamente em movimento, é necessário utilizar um procedimento para a identificação da região que contém o fluido e a superfície livre. Para isto, sinaliza-se todas as células da malha segundo suas características:

I $\rightarrow$ Células de entrada (injeção): células que simulam a entrada do fluido na região do domínio;

$\mathrm{F} \rightarrow$ Células de fronteira: células que definem o contorno rígido, de modo que possam ser impostas as condiçōes de contorno;

$\mathrm{V} \rightarrow$ Células vazias: trata-se de células que não contém fluido;

$S \rightarrow$ Células de superfície: células que contém fluido com pelo menos uma célula vizinha vazia e definem a posição da superfície livre;

$\mathrm{C} \rightarrow$ Células cheias: células que contém o fluido e não têm contato com células vazias;

$\mathrm{E} \rightarrow$ Células de saída (ejeção): células que simulam a saída do fluido da região de domínio.

Para visualizar melhor a sinalização das células, observe a figura 2.1 [3]. Pạa - a simplificaçāo no entendimento da figura opta-se pela supressão da nomenclatura das células vazias $(\mathrm{V})$.

Conforme o problema em questão, uma célula $\mathrm{C}$ pode tornar-se uma célula $\mathrm{S}$ e então $V$, ou vice-versa. 


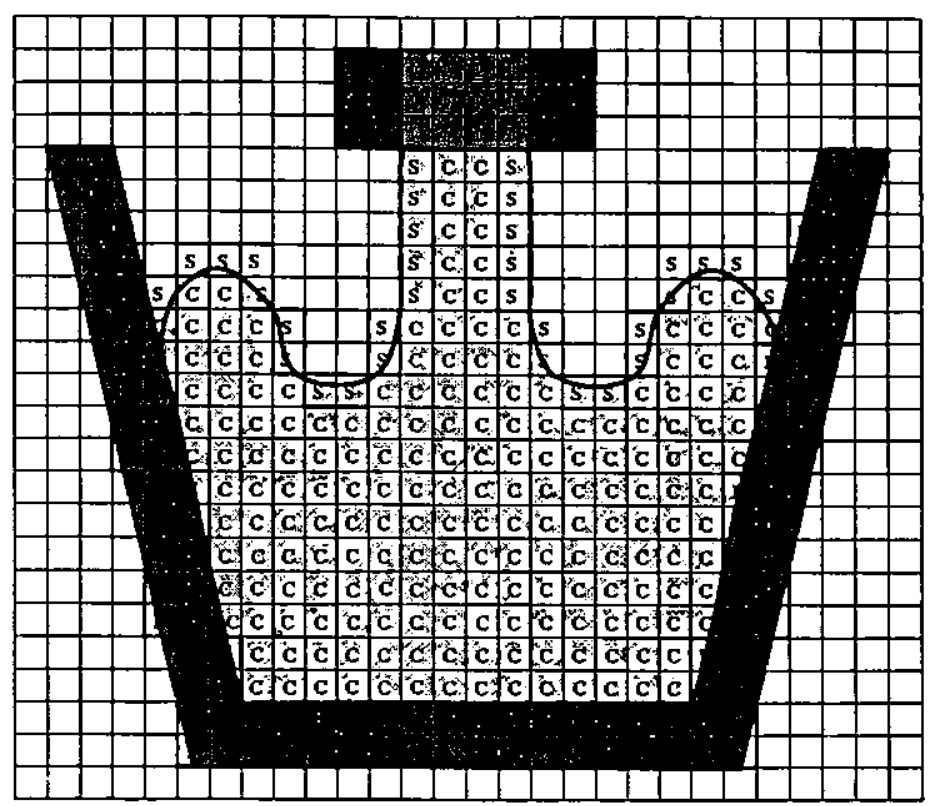

Figura 2.1: Domínio, malha e células

\subsection{Condições de Contorno na Superfície Livre}

Considera-se três tipos de orientação para a superfície livre: horizontal, vertical e inclinada à 45 graus. Supondo-se que o espaçamento da malha seja suficientemente pequeno para que a superfície livre intercepte uma célula em dois lados, obtém-se os seguintes casos:

(i) Células de superfície (S) com apenas um lado em comum com uma célula vazia (V)

Neste caso, assumi-se que a superfície será horizontal ou vertical.

Considere a figura 2.2 como exemplo.

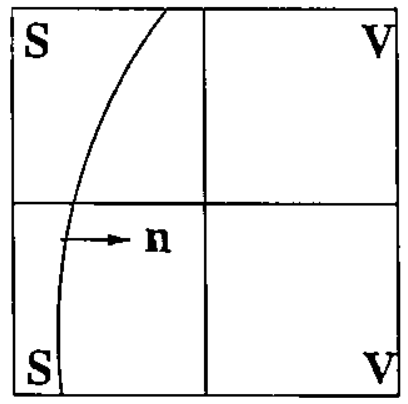

Figura 2.2: Célula (S) com o lado direito em contato com uma célula (V)

Substituindo $n=(1,0)$ e $m=(0,1)$ em 1.38 e 1.39 obtém-se: 


$$
\begin{gathered}
-p+\frac{2 \nu(q)}{\operatorname{Re}}\left(\frac{\partial \dot{u}}{\partial x}\right)=0 \\
\frac{\partial u}{\partial y}+\frac{\partial v}{\partial x}=0
\end{gathered}
$$

Utilizando-se 2.9 e a equação da continuidade, a razão de cisalhamento se reduz a:

$$
q=2\left|\frac{\partial u}{\partial x}\right|
$$

São tratados de.forma análoga outros casos de células (S) com uma célula vizinha vazia.

(ii) Células de superfície (S) com dois lados em contato com uma célula vazia (V)

Neste caso, assumi-se que o vetor normal seja inclinado de 45 graus entre os lados voltados para as células vazias. Fazendo $n=\left( \pm \frac{\sqrt{2}}{2}, \pm \frac{\sqrt{2}}{2}\right)$, as condiçōes de contorno 1.38 e 1.39 tornam-se

$$
\begin{gathered}
-p \pm \frac{\nu(q)}{R e}\left(\frac{\partial u}{\partial y}+\frac{\partial v}{\partial x}\right)=0 \\
\frac{\partial u}{\partial x}-\frac{\partial v}{\partial y}=0
\end{gathered}
$$

O sinal de 2.10 será escolhido de acordo com a posição do vetor normal.-.- Por exemplo, considera-se a célula como mostra a figura 2.3 .

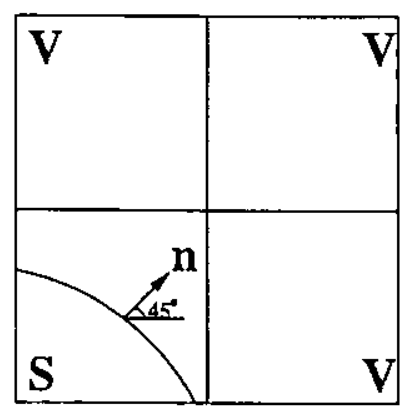

Figura 2.3: Célula (S) com dois lados em contato com células (V)

Decorre de 2.11 e da equação da continuidade que a razão de cisalhamento se reduz

$$
q=\left|\frac{\partial u}{\partial y}+\frac{\partial v}{\partial x}\right|
$$


São tratados de forma análoga outros casos de células de superfície com duas células vizinhas vazias.

(iii) Células de superfície (S) com três lados em contato com células vazias (V) ou dois lados opostos em contato com células vazias

Para estas células, a pressão é tomada nula e a velocidade é ajustada a fim de que $\nabla . u=0$ na célula S. A razão de cisalhamento $q_{i, j}$ é zero.

Se tais células aparecerem, é necessário que a malha seja refinada. 


\section{Capítulo 3}

\section{Discretizações}

\subsection{Considerações Iniciais}

As equações de Navier-Stokes formam um sistema acoplado não-linear de equações diferenciais parciais. Soluções analíticas são obtidas para geometrias e condiçōes de escoamento extremamente limitadas em termo de aplicação. Portanto o único caminho viável é o uso de soluções numéricas. Um método numérico consiste em [19]:

- discretizar o domínio, ou seja, distribuir um certo número de pontos discretos no espaço onde a solução é dada;

- discretizar as equações, transformando o sistema de equações diferenciais em um sistema de equações algébricas.

A partir daí, recorre-se a técnicas de solução das equações algébricas.

\subsection{Discretização do Domínio}

Para a realização da aproximação numérica é necessária a discretização do domínio de interesse. A solução é computada no interior do domínio e as fronteiras representam o problema em questão. As possibilidades de escolha da posição dos pontos discretos onde a solução será calculada, e a forma de aplicação dà̀ leis de conservação nessa malha, são diversas.

Uma malha é dita estruturada se cada ponto da malha pode ser localizado por uma sequiência de índices ordenados. Seja uma malha bidimensional cartesiana. O ponto $P(x, y)$ tem como vizinhança: $P(x+\Delta x, y), P(x-\Delta x, y), P(x, y+\Delta y)$ e $P(x, y-\Delta y)$. 
Um sistema estruturado permite associar índices tal que: $P_{i, j}=P(x, y)$ e seus vizinhos são associados respectivamente a $P_{i+1, j i}, P_{i-1, j}, P_{i, j+1}$ e. $P_{i, j-1}$. Dessa forma a única informação a ser armazenada é a relação dos índices com as posições dos pontos.

Malha diferenciada é uma malha estruturada em que as variáveis são posicionadas em diferentes pontos. Por exemplo, a malha MAC possui as componentes da velocidade localizadas nos centros das faces da célula e a pressão é avaliada no centro, como mostra a figura $3.1[8]$ :

O uso de malhas diferenciadas impede o aparecimento de soluções oscilatórias,

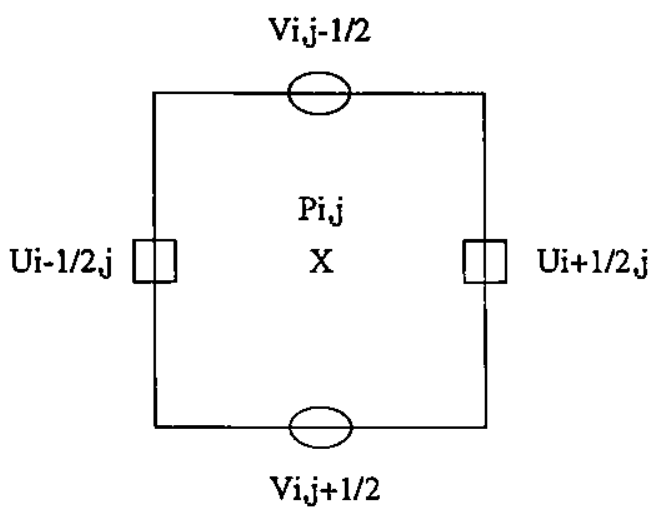

Figura 3.1: Célula MAC

em particular para a pressão, que podem ocorrer se diferenças centrais são utilizadas nas discretizações das derivadas [7] [6]. Soluções Oscilatórias representam a manifestação de duas soluções diferentes para a pressão, associadas a pontos alternados da malha, geradas pelo uso de diferenças centrais numa malha não diferenciada. A oscilação torna-se ainda pior se o número de Reynolds for alto para os termos dissipativos.

Uma vantagem adicional da malha diferenciada é que a equação de Poisson para a pressão automaticamente é satisfeita, sem a necessidade de nenhuma condição de fronteira adicional, evitando-se assim ajustes no lado direito da equação.

Entretanto, algumas desvantagens são observadas:

- no emprego de malhas diferenciadas há dificuldades em relação a implementação;

- a extensão para sistemas de coordenadas gerais, se a malha não for retangular, torna-se um pouco complexa.

Os métodos de discretizações por aproximações polinomiais são mais eficientes se aplicados em um sistema de coordenadas cartesianas com espaçamento constante. Entre- 
tanto, a maioria das aplicações de interesse ocorrem justamente em dómínios complexos que em geral não são retangulares. Assim, é necessário transformar o domínio físico não-retangular em um domínio computacional cartesiano, onde os pontos da malha são igualmente espaçados. Essa transformação permite alinhar uma das coordenadas a superfície de interesse facilitando a imposição das condições de contorno. A relação entre o espaço físico e o computacional, em duas dimensões, é dada pela relação:

$$
\begin{gathered}
\xi=\xi(x, y) \\
\eta=\eta(x, y) .
\end{gathered}
$$

Em alguns casos é impossível encontrar uma transformação de coordenadas capaz de mapear o domínio em um retângulo (ou em um cubo em 3D), um artíficio bastante utilizado é a subdivisão do domínio em diversos blocos, onde cada um pode ser transformado. Nas interfaces dos blocos a solução deverá ser transmitida de maneira semelhante as condições de contorno.

\subsection{Discretização das Equações}

Uma vez discretizado o domínio, para se obter a solução numérica é necessário transformar o sistema de equações diferenciais parciais em um sistema de equações algébricas. Esse processo é feito através das aproximações das equações assuminḍo que as soluções têm forma polinomial entre pontos adjacentes da malha.

\subsection{O Método das Diferenças Finitas}

Este método consiste em aproximar as derivadas por fórmulas de diferenças [21]. Expandindo uma função $F(x)$ em Série de Taylor em torno de um valor $\left(x_{i}\right)$, onde $i=0, \cdots, n$ representam pontos discretos na reta real, tem-se:

$$
F\left(x_{i}+\Delta x\right)=F\left(x_{i}\right)+\Delta x\left(\frac{\partial F}{\partial x}\right)_{x_{i}}+\frac{\Delta x^{2}}{2}\left(\frac{\partial^{2} F}{\partial x^{2}}\right)_{x_{i}}+\cdots
$$

e

$$
F\left(x_{i}-\Delta x\right)=F\left(x_{i}\right)-\Delta x\left(\frac{\partial F}{\partial x}\right)_{x_{i}}+\frac{\Delta x^{2}}{2}\left(\frac{\partial^{2} F}{\partial x^{2}}\right)_{x_{i}}+\cdots
$$


Subtraindo as equações, obtém-se:

$$
\left(\frac{\partial F}{\partial x}\right)_{x_{i}} \cong \frac{F\left(x_{i}+\Delta x\right)-F\left(x_{i}-\Delta x\right)}{2 \Delta x}
$$

Adicionando-se tem-se:

$$
\left(\frac{\partial^{2} F}{\partial x^{2}}\right)_{x_{i}} \cong \frac{F\left(x_{i}+\Delta x\right)-2 F\left(x_{i}\right)+F\left(x_{i}-\Delta x\right)}{\Delta x^{2}}
$$

Essas đuas expressões são aproximações de ordem $O\left(\Delta x^{2}\right)$ da primeira e da segunda derivada centradas em $\left(x_{i}\right)$.

Utilizando outras combinações de expansōes em série pode-se deduzir uma variedade de fórmulas de diferenças.

O método de discretização empregado pode ser explícito ou implícito, sendo que os explícitos estão sujeitos a algumas restrições de estabilidade, enquanto que os implícitos são incondicionalmente estáveis.

\subsection{A Discretização da Equação de Momento}

As equaçōes de Navier-Stokes e a da continuidade, nas formas adimensionais, são dadas por 1.23, 1.24 e 1.25. A discretização dessas equações é feita sobre malhas diferenciadas, onde a equação 1.23 é discretizada no ponto $\left(i+\frac{1}{2}, j\right), 1.24$ no ponto $\left(i, j+\frac{1}{2}\right)$ e 1.25 no ponto $(i, j)$, como ilustra a figura $3.2[11]$.

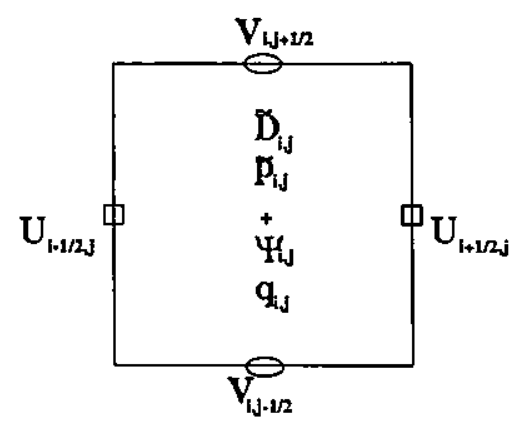

Figura 3.2: Configuração de uma célula

As aproximações para as derivadas temporais são feitas utilizando diferenças avançadas de primeira ordem. Os termos convectivos e os gradientes de pressão são aproximados usando-se diferenças centrais: 


$$
\begin{gathered}
{\left[\frac{\partial \bar{u}}{\partial t}\right]_{i+\frac{1}{2}, j}=\frac{\tilde{u}_{i+\frac{1}{2}, j}^{n+1}-\tilde{u}_{i+\frac{1}{2}, j}^{n}}{\delta t}} \\
{\left[\frac{\partial \tilde{v}}{\partial t}\right]_{i, j+\frac{1}{2}}=\frac{\tilde{v}_{i, j+\frac{1}{2}}^{n+1}-\tilde{v}_{i, j+\frac{1}{2}}^{n}}{\delta t}} \\
{\left[\frac{\partial \tilde{p}}{\partial x}\right]_{i+\frac{1}{2}, j}=\frac{\tilde{p}_{i+1, j}-\tilde{p}_{i, j}}{\delta x}} \\
{\left[\frac{\partial \bar{p}}{\partial y}\right]_{i, j+\frac{1}{2}}=\frac{\tilde{p}_{i, j+1}-\tilde{p}_{i, j}}{\delta y}} \\
{\left[\frac{\partial u^{2}}{\partial x}\right]_{i+\frac{1}{2}, j}=\frac{u_{i+1, j}^{2}-u_{i, j}^{2}}{\delta x}} \\
{\left[\frac{\partial v^{2}}{\partial y}\right]_{i, j+\frac{1}{2}}=\frac{v_{i, j+1}^{2}-v_{i, j}^{2}}{\delta y}}
\end{gathered}
$$

onde os valores $(u)^{2}$ e $(v)^{2}$ sāo aproximados utilizando a forma ZIP [24]:

$$
\begin{gathered}
\left(u_{i+1, j}\right)^{2}=u_{i+\frac{3}{2}, j} \cdot u_{i+\frac{1}{2}, j} \\
\left(u_{i, j}\right)^{2}=u_{i+\frac{1}{2}, j} \cdot u_{i-\frac{1}{2}, j} \\
\left(v_{i, j+1}\right)^{2}=v_{i, j+\frac{3}{2}} \cdot v_{i, j+\frac{1}{2}} \\
\left(v_{i, j}\right)^{2}=v_{i, j+\frac{1}{2}} \cdot v_{i, j-\frac{1}{2}} .
\end{gathered}
$$

Então:

$$
\left[\frac{\partial u^{2}}{\partial x}\right]_{i+\frac{1}{2}, j}=\frac{u_{i+\frac{3}{2}, j} \cdot u_{i+\frac{1}{2}, j}-u_{i+\frac{1}{2}, j} \cdot u_{i-\frac{1}{2}, j}}{\delta x}
$$

e

$$
\left[\frac{\partial v^{2}}{\partial y}\right]_{i, j+\frac{1}{2}}=\frac{v_{i, j+\frac{3}{2}} \cdot v_{i, j+\frac{1}{2}}-v_{i, j+\frac{1}{2}} \cdot v_{i, j-\frac{1}{2}}}{\delta y}
$$

Os termos de fluxo $\frac{\partial(u v)}{\partial y}$ e $\frac{\partial(u v)}{\partial x}$ são aproximados por diferenças centrais e os valores das variáveis em pontos indeterminados nas malhas são calculados como as médias aritméticas dos valores nas faces das células: 


$$
\left[\frac{\partial(u v)}{\partial y}\right]_{i+\frac{1}{2}, j}=\frac{(u v)_{i+\frac{1}{2}, j+\frac{1}{2}}-(u v)_{i+\frac{1}{2}, j-\frac{1}{2}}}{\delta y}
$$

onde

$$
\begin{aligned}
& (u v)_{i+\frac{1}{2}, j+\frac{1}{2}}=\frac{\left(u_{i+\frac{1}{2}, j}+u_{i+\frac{1}{2}, j+1}\right)}{2} \cdot \frac{\left(v_{i, j+\frac{1}{2}}+v_{i+1, j+\frac{1}{2}}\right)}{2} \\
& (u v)_{i+\frac{1}{2}, j-\frac{1}{2}}=\frac{\left(u_{i+\frac{1}{2}, j}+u_{i+\frac{1}{2}, j-1}\right)}{2} \cdot \frac{\left(v_{i, j-\frac{1}{2}}+v_{i+1, j-\frac{1}{2}}\right)}{2} .
\end{aligned}
$$

Então

$$
\begin{gathered}
{\left[\frac{\partial(u v)}{\partial y}\right]_{i+\frac{1}{2}, j}=\frac{1}{\delta y}\left[\frac{\left(u_{i+\frac{1}{2}, j}+u_{i+\frac{1}{2}, j+1}\right) \cdot\left(v_{i, j+\frac{1}{2}}+v_{i+1, j+\frac{1}{2}}\right)}{4}-\right.} \\
\left.\frac{\left(u_{i+\frac{1}{2}, j}+u_{i+\frac{1}{2}, j-1}\right) \cdot\left(v_{i, j-\frac{1}{2}}+v_{i+1, j-\frac{1}{2}}\right)}{4}\right]
\end{gathered}
$$

Logo, .

$$
\begin{gathered}
{\left[\frac{\partial(u v)}{\partial y}\right]_{i+\frac{1}{2}, j}=\frac{1}{4}\left[\frac{u_{i+\frac{1}{2}, j}\left(v_{i, j+\frac{1}{2}}+v_{i+1, j+\frac{1}{2}}-v_{i, j-\frac{1}{2}}-v_{i+1, j-\frac{1}{2}}\right)}{\delta y}+\right.} \\
\left.\frac{u_{i+\frac{1}{2}, j+1}\left(v_{i, j+\frac{1}{2}}+v_{i+1, j+\frac{1}{2}}\right)-u_{i+\frac{1}{2}, j-1}\left(v_{i, j-\frac{1}{2}}+v_{i+1, j-\frac{1}{2}}\right)}{\delta y}\right] \\
{\left[\frac{\partial(u v)}{\partial x}\right]_{i, j+\frac{1}{2}}=\frac{(u v)_{i+\frac{1}{2}, j+\frac{1}{2}}-(u v)_{i-\frac{1}{2}, j+\frac{1}{2}}}{\delta x},}
\end{gathered}
$$

onde

$$
\begin{aligned}
& (u v)_{i+\frac{1}{2}, j+\frac{1}{2}}=\frac{\left(u_{i+\frac{1}{2}, j}+u_{i+\frac{1}{2}, j+1}\right)}{2} \cdot \frac{\left(v_{i, j+\frac{1}{2}}+v_{i+1, j+\frac{1}{2}}\right)}{2} \\
& (u v)_{i-\frac{1}{2}, j+\frac{1}{2}}=\frac{\left(u_{i-\frac{1}{2}, j+1}+u_{i-\frac{1}{2}, j}\right)}{2} \cdot \frac{\left(v_{i, j+\frac{1}{2}}+v_{i-1, j+\frac{1}{2}}\right)}{2} .
\end{aligned}
$$


Então

$$
\begin{gathered}
{\left[\frac{\partial(u v)}{\partial x}\right]_{i, j+\frac{1}{2}}=\frac{1}{4}\left[\frac{\left(u_{i+\frac{1}{2}, j}+u_{i+\frac{1}{2}, j+1}\right) \cdot\left(v_{i, j+\frac{1}{2}}+v_{i+1, j+\frac{1}{2}}\right)}{\delta x}-\right.} \\
\left.\frac{\left(u_{i-\frac{1}{2}, j+1}+u_{i-\frac{1}{2}, j}\right) \cdot\left(v_{i, j+\frac{1}{2}}+v_{i-1, j+\frac{1}{2}}\right)}{\delta x}\right] \\
{\left[\frac{\partial(u v)}{\partial x}\right]_{i, j+\frac{1}{2}}=\frac{1}{4}\left[\frac{v_{i, j+\frac{1}{2}}\left(u_{i+\frac{1}{2}, j}+u_{i+\frac{1}{2}, j+1}-u_{i-\frac{1}{2}, j}-u_{i-\frac{1}{2}, j+1}\right)}{\delta x}+\right.} \\
\left.\frac{v_{i+1, j+\frac{1}{2}}\left(u_{i+\frac{1}{2}, j}+u_{i+\frac{1}{2}, j+1}\right)-v_{i-1, j+\frac{1}{2}}\left(u_{i-\frac{1}{2}, j}+u_{i-\frac{1}{2}, j+1}\right)}{\delta x}\right] .
\end{gathered}
$$

Discretizando o termo $\frac{1}{R e} \nu(q) \frac{\partial}{\partial y}\left(\frac{\partial u}{\partial y}-\frac{\partial v}{\partial x}\right)$ da equação 1.28 , obtém-se:

$$
\frac{\partial}{\partial y}\left(\frac{\partial u}{\partial y}\right)_{i+\frac{1}{2}, j}=\frac{\left[\frac{\partial u}{\partial y}\right]_{i+\frac{1}{2}, j+\frac{1}{2}}-\left[\frac{\partial u}{\partial y}\right]_{i+\frac{1}{2}, j-\frac{1}{2}}}{\delta y}=\left[\frac{\partial^{2} u}{\partial y^{2}}\right]_{i+\frac{1}{2}, j}
$$

Diferença centrada de segunda ordem:

$$
\begin{gathered}
{\left[\frac{\partial^{2} u}{\partial y^{2}}\right]_{i+\frac{1}{2}, j}=\frac{u_{i+\frac{1}{2}, j+1}-2 u_{i+\frac{1}{2}, j}+u_{i+\frac{1}{2}, j-1}}{(\delta y)^{2}}} \\
\frac{\partial}{\partial y}\left[\frac{\partial v}{\partial x}\right]_{i+\frac{1}{2}, j}=\frac{\partial}{\partial y}\left[\frac{v_{i+1, j}-v_{i, j}}{\delta x}\right]_{i+\frac{1}{2}, j} \\
\frac{\partial}{\partial y}\left[\frac{\partial v}{\partial x}\right]_{i+\frac{1}{2}, j}=\frac{\left[\frac{\partial v}{\partial y}\right]_{i+1, j}-\left[\frac{\partial v}{\partial y}\right]_{i, j}}{\delta x} \\
\frac{\partial}{\partial y}\left[\frac{\partial v}{\partial x}\right]_{i+\frac{1}{2}, j}=\frac{v_{i+1, j+\frac{1}{2}}-v_{i+1, j-\frac{1}{2}}-v_{i, j+\frac{1}{2}}+v_{i, j-\frac{1}{2}}}{\delta x \delta y} .
\end{gathered}
$$


Discretizando o termo $\frac{1}{R e} \nu(q) \frac{\partial}{\partial x}\left(\frac{\partial u}{\partial y}-\frac{\partial v}{\partial x}\right)$ da equação 1.29 , obtém-se:

$$
\frac{\partial}{\partial x}\left[\frac{\partial u}{\partial y}\right]_{i, j+\frac{1}{2}}=\frac{\left[\frac{\partial u}{\partial y}\right]_{i+\frac{1}{2}, j+\frac{1}{2}}-\left[\frac{\partial u}{\partial y}\right]_{i-\frac{1}{2}, j+\frac{1}{2}}}{\delta x}
$$

onde

$$
\begin{gathered}
{\left[\frac{\partial u}{\partial y}\right]_{i+\frac{1}{2}, j+\frac{1}{2}}=\frac{u_{i+\frac{1}{2}, j+1}-u_{i+\frac{1}{2}, j}}{\delta y}} \\
{\left[\frac{\partial u}{\partial y}\right]_{i-\frac{1}{2}, j+\frac{1}{2}}=\frac{u_{i-\frac{1}{2}, j+1}-u_{i-\frac{1}{2}, j}}{\delta y} .}
\end{gathered}
$$

Então:

$$
\begin{gathered}
\frac{\partial}{\partial x}\left[\frac{\partial u}{\partial y}\right]_{i, j+\frac{1}{2}}=\frac{u_{i+\frac{1}{2}, j+1}-u_{i+\frac{1}{2}, j}-u_{i-\frac{1}{2}, j+1}+u_{i-\frac{1}{2}, j}}{\delta x \delta y} \\
\frac{\partial}{\partial x}\left[\frac{\partial v}{\partial x}\right]_{i, j+\frac{1}{2}}=\frac{\left[\frac{\partial v}{\partial x}\right]_{i+\frac{1}{2}, j+\frac{1}{2}}-\left[\frac{\partial v}{\partial x}\right]_{i-\frac{1}{2}, j+\frac{1}{2}}}{\delta x}=\left[\frac{\partial^{2} v}{\partial x^{2}}\right]_{i, j+\frac{1}{2}}
\end{gathered}
$$

Diferença centrada de segunda ordem:

$$
\left[\frac{\partial^{2} v}{\partial x^{2}}\right]_{i, j+\frac{1}{2}}=\frac{v_{i+1, j+\frac{1}{2}}-2 v_{i, j+\frac{1}{2}}+v_{i-1, j+\frac{1}{2}}}{(\delta x)^{2}}
$$

Discretização do termo $\frac{1}{R e}\left[2 \frac{\partial u}{\partial x} \frac{\partial \nu(q)}{\partial x}+\left(\frac{\partial u}{\partial y}+\frac{\partial v}{\partial x}\right) \frac{\partial \nu(q)}{\partial y}+\frac{1}{F r^{2}} g_{x}\right]$, da equação 1.28 . obtém-se as seguintes aproximaçōes para as derivadas que não contém $\nu$-derivadas :

$$
\begin{gathered}
{\left[\frac{\partial u}{\partial x}\right]_{i+\frac{1}{2}, j}=\frac{u_{i+\frac{3}{2}, j}-u_{i-\frac{1}{2}, j}}{2 \delta x}} \\
{\left[\frac{\partial u}{\partial y}\right]_{i+\frac{1}{2}, j}=\frac{u_{i+\frac{1}{2}, j+1}-u_{i+\frac{1}{2}, j-1}}{2 \delta y} .}
\end{gathered}
$$




$$
\left[\frac{\partial v}{\partial x}\right]_{i+\frac{1}{2}, j}=\frac{v_{i+1, j}-v_{i, j}}{\delta x}
$$

onde

$$
\begin{gathered}
v_{i+1, j}=\frac{v_{i+1, j+\frac{1}{2}}+v_{i+1, j-\frac{1}{2}}}{2} \\
v_{i, j}=\frac{v_{i, j+\frac{1}{2}}+v_{i, j-\frac{1}{2}}}{2} .
\end{gathered}
$$

Entāo

$$
\left[\frac{\partial v}{\partial x}\right]_{i+\frac{1}{2}, j}=\frac{v_{i+1, j+\frac{1}{2}}+v_{i+1, j-\frac{1}{2}}-v_{i, j+\frac{1}{2}}-v_{i, j-\frac{1}{2}}}{2 \delta x}
$$

Discretizando o termo $\frac{1}{R e}\left[\left(\frac{\partial u}{\partial y}+\frac{\partial v}{\partial x}\right) \frac{\partial \nu(q)}{\partial x}+2 \frac{\partial v}{\partial y} \frac{\partial \nu(q)}{\partial y}\right]+\frac{1}{F r^{2}} g_{y}$ da equação 1.29 , obtém-se as seguintes aproximações para as derivadas que não contém $\nu$-derivadas :

$$
\left[\frac{\partial u}{\partial y}\right]_{i, j+\frac{1}{2}}=\frac{u_{i, j+1}-u_{i, j}}{\delta y}
$$

onde

$$
\begin{aligned}
u_{i, j+1} & =\frac{u_{i+\frac{1}{2}, j+1}+u_{i-\frac{1}{2}, j+1}}{2} \\
u_{i, j} & =\frac{u_{i+\frac{1}{2}, j}+u_{i-\frac{1}{2}, j}}{2} .
\end{aligned}
$$

Então

$$
\begin{gathered}
{\left[\frac{\partial u}{\partial y}\right]_{i, j+\frac{1}{2}}=\frac{u_{i+\frac{1}{2}, j+1}+u_{i-\frac{1}{2}, j+1}-u_{i+\frac{1}{2}, j}-u_{i-\frac{1}{2}, j}}{2 \delta y}} \\
{\left[\frac{\partial v}{\partial x}\right]_{i, j+\frac{1}{2}}=\frac{v_{i+1, j+\frac{1}{2}}-v_{i-1, j+\frac{1}{2}}}{2 \delta x}} \\
{\left[\frac{\partial v}{\partial y}\right]_{i, j+\frac{1}{2}}=\frac{v_{i, j+\frac{3}{2}}-v_{i, j-\frac{1}{2}}}{2 \delta y} .} \\
\cdot 31
\end{gathered} .
$$


Para a aproximação de cada termo da equação da razão de cisalhamento 1.30 segue que:

Os termos $\frac{\partial u}{\partial x}$ e $\frac{\partial v}{\partial y}$ são discretizados por diferenças centrais, ou seja:

$$
\begin{aligned}
& {\left[\frac{\partial u}{\partial x}\right]_{i, j}=\frac{u_{i+\frac{1}{2}, j}-u_{i-\frac{1}{2}, j}}{\delta x}} \\
& {\left[\frac{\partial v}{\partial y}\right]_{i, j}=\frac{v_{i, j+\frac{1}{2}}-v_{i, j-\frac{1}{2}}}{\delta y}} \\
& {\left[\frac{\partial u}{\partial y}\right]_{i, j}=\frac{u_{i, j+\frac{1}{2}}-u_{i, j-\frac{1}{2}}}{\delta y},}
\end{aligned}
$$

onde

$$
\begin{gathered}
u_{i, j+\frac{1}{2}}=\frac{u_{i+\frac{1}{2}, j+\frac{1}{2}}+u_{i-\frac{1}{2}, j+\frac{1}{2}}}{2}=\frac{1}{4}\left[u_{i+\frac{1}{2}, j+1}+u_{i+\frac{1}{2}, j}+u_{i-\frac{1}{2}, j+1}+u_{i-\frac{1}{2}, j}\right] \\
-. \\
u_{i, j-\frac{1}{2}}=\frac{u_{i+\frac{1}{2}, j-\frac{1}{2}}+u_{i-\frac{1}{2}, j-\frac{1}{2}}}{2}=\frac{1}{4}\left[u_{i+\frac{1}{2}, j}+u_{i+\frac{1}{2}, j-1}+u_{i-\frac{1}{2}, j}+u_{i-\frac{1}{2}, j-1}\right] .
\end{gathered}
$$

Logo:

$$
\left[\frac{\partial u}{\partial y}\right]_{i, j}=\frac{1}{4}\left[\frac{u_{i+\frac{1}{2}, j+1}+u_{i-\frac{1}{2}, j+1}-u_{i+\frac{1}{2}, j-1}-u_{i-\frac{1}{2}, j-1}}{\delta y}\right]
$$

Analogamente, tem-se:

$$
\left[\frac{\partial v}{\partial x}\right]_{i, j}=\frac{v_{i+\frac{1}{2}, j}-v_{i-\frac{1}{2}, j}}{\delta x}
$$

onde

$$
\begin{aligned}
& v_{i+\frac{1}{2}, j}=\frac{v_{i+\frac{1}{2}, j+\frac{1}{2}}+v_{i+\frac{1}{2}, j-\frac{1}{2}}}{2}=\frac{1}{4}\left[v_{i, j+\frac{1}{2}}+v_{i+1, j+\frac{1}{2}}+v_{i, j-\frac{1}{2}}+v_{i+1, j-\frac{1}{2}}\right] \\
& v_{i-\frac{1}{2}, j}=\frac{v_{i-\frac{1}{2}, j+\frac{1}{2}}+v_{i-\frac{1}{2}, j-\frac{1}{2}}}{2}=\frac{1}{4}\left[v_{i-1, j+\frac{1}{2}}+v_{i, j+\frac{1}{2}}+v_{i-1, j-\frac{1}{2}}+v_{i, j-\frac{1}{2}}\right]
\end{aligned}
$$


Então:

$$
\left[\frac{\partial v}{\partial x}\right]_{i, j}=\frac{1}{4}\left[\frac{v_{i+1, j+\frac{1}{2}}+v_{i+1, j-\frac{1}{2}}-v_{i-1, j+\frac{1}{2}}-v_{i-1, j-\frac{1}{2}}}{\delta x}\right]
$$

E portanto, obtém-se a razão de cisalhamento $q_{i, j}$ discretizada:

$$
\begin{gathered}
q_{i, j}=\left[2\left(\frac{u_{i+\frac{1}{2}, j}-u_{i-\frac{1}{2}, j}}{\delta x}\right)^{2}+2\left(\frac{v_{i, j+\frac{1}{2}}-v_{i, j-\frac{1}{2}}}{\delta y}\right)^{2}+\right. \\
\left(\frac{1}{4}\left[\frac{u_{i+\frac{1}{2}, j+1}+u_{i-\frac{1}{2}, j+1}-u_{i+\frac{1}{2}, j-1}-u_{i-\frac{1}{2}, j-1}}{\delta y}\right]+\right. \\
\left.\left.\frac{1}{4}\left[\frac{v_{i+1, j+\frac{1}{2}}+v_{i+1, j-\frac{1}{2}}-v_{i-1, j+\frac{1}{2}}-v_{i-1, j-\frac{1}{2}}}{\delta x}\right]\right)^{2}\right]^{\frac{1}{2}}
\end{gathered}
$$

Aproxima-se as $\nu$-derivadas da seguinte forma:

$$
\begin{gathered}
{\left[\frac{\partial \nu}{\partial x}\right]_{i+\frac{1}{2}, j}=\frac{\nu\left(q_{i+1, j}\right)-\nu\left(q_{i, j}\right)}{\delta x}} \\
{\left[\frac{\partial \nu}{\partial y}\right]_{i, j+\frac{1}{2}}=\frac{\nu\left(q_{i, j+1}\right)-\nu\left(q_{i, j}\right)}{\delta y} .} \\
{\left[\frac{\partial \nu}{\partial x}\right]_{i, j+\frac{1}{2}}=\frac{\nu\left(q_{i+\frac{1}{2}, j+\frac{1}{2}}\right)-\nu\left(q_{i-\frac{1}{2}, j+\frac{1}{2}}\right)}{\delta x}}
\end{gathered}
$$

- onde

$$
\begin{aligned}
& q_{i+\frac{1}{2}, j+\frac{1}{2}}=\frac{q_{i+\frac{1}{2}, j+1}+q_{i+\frac{1}{2}, j}}{2}=\frac{1}{4}\left[q_{i, j+1}+q_{i+1, j+1}+q_{i, j}+q_{i+1, j}\right] \\
& q_{i-\frac{1}{2}, j+\frac{1}{2}}=\frac{q_{i-\frac{1}{2}, j+1}+q_{i-\frac{1}{2}, j}}{2}=\frac{1}{4}\left[q_{i-1, j+1}+q_{i, j+1}+q_{i-1, j}+q_{i, j}\right] .
\end{aligned}
$$




$$
\left[\frac{\partial \nu}{\partial y}\right]_{i+\frac{1}{2}, j}=\frac{\nu\left(q_{i+\frac{1}{2}, j+\frac{1}{2}}\right)-\nu\left(q_{i+\frac{1}{2}, j-\frac{1}{2}}\right)}{\delta y}
$$

onde

$$
q_{i+\frac{1}{2}, j-\frac{1}{2}}=\frac{1}{4}\left[q_{i, j}+q_{i+1, j}+q_{i, j-1}+q_{i+1, j-1}\right] .
$$

De acordo com as aproximações desenvolvidas neste capítulo, obtém-se a equação de momento 1.26 discretizada:

$$
\begin{gathered}
\frac{\tilde{u}_{i+\frac{1}{2}, j}^{n+1}-\tilde{u}_{i+\frac{1}{2}, j}^{n}}{\delta t}=-\left(\frac{\tilde{p}_{i+1, j}-\vec{p}_{i, j}}{\delta x}\right)-\left(\frac{u_{i+\frac{3}{2}, j} \cdot u_{i+\frac{1}{2}, j}-u_{i+\frac{1}{2}, j} \cdot u_{i-\frac{1}{2}, j}}{\delta x}\right)- \\
\frac{1}{4}\left[\frac{u_{i+\frac{1}{2}, j}\left(v_{i, j+\frac{1}{2}}+v_{i+1, j+\frac{1}{2}}-v_{i, j-\frac{1}{2}}-v_{i+1, j-\frac{1}{2}}\right)}{\delta y}+\right. \\
\left.\frac{u_{i+\frac{1}{2}, j+1}\left(v_{i, j+\frac{1}{2}}+v_{i+1, j+\frac{1}{2}}\right)-u_{i+\frac{2}{2}, j-1}\left(v_{i, j-\frac{1}{2}}+v_{i+1, j-\frac{1}{2}}\right)}{\delta y}\right]+ \\
\left(\frac { v _ { i + 1 , j + \frac { 1 } { 2 } } - v _ { i + 1 , j - \frac { 1 } { 2 } } + v _ { i , j - \frac { 1 } { 2 } } - v _ { i , j + \frac { 1 } { 2 } } } { \delta x \delta y } \nu \left(q _ { i + \frac { 1 } { 2 } , j } \left[\left(\frac{u_{i+\frac{1}{2}, j+1}-2 u_{i+\frac{1}{2}, j}+u_{i+\frac{1}{2}, j-1}}{(\delta y)^{2}}\right)-\frac{1}{R e}\left[2\left(\frac{u_{i+\frac{3}{2}, j}-u_{i-\frac{1}{2}, j}}{2 \delta x}\right)\left[\frac{\partial \nu}{\partial x}\right]_{i+\frac{1}{2}, j}+\right.\right.\right.\right. \\
\left.\left[\left(\frac{u_{i+\frac{1}{2}, j+1}-u_{i+\frac{1}{2}, j-1}}{2 \delta y}\right)+\left(\frac{v_{i+1, j+\frac{1}{2}}+v_{i+1, j-\frac{1}{2}}-v_{i, j+\frac{1}{2}}-v_{i, j-\frac{1}{2}}}{2 \delta x}\right)\right]\left[\frac{\partial \nu}{\partial y}\right]_{i+\frac{1}{2}, j}\right]+ \\
\frac{1}{F r^{2}} g_{x}
\end{gathered}
$$




$$
\begin{aligned}
& \frac{\tilde{v}_{i, j+\frac{1}{2}}^{n+1}-\tilde{v}_{i, j+\frac{1}{2}}^{\mathrm{n}}}{\delta t}=-\left(\frac{\tilde{p}_{i, j+1}-\tilde{p}_{i, j}}{\delta y}\right)-\left(\frac{v_{i, j+\frac{3}{2}} \cdot v_{i, j+\frac{1}{2}}-v_{i, j+\frac{1}{2}} \cdot v_{i, j-\frac{1}{2}}}{\delta y}\right)- \\
& \frac{1}{4}\left[\frac{v_{i, j+\frac{1}{2}}\left(u_{i+\frac{1}{2}, j}+u_{i+\frac{1}{2}, j+1}-u_{i-\frac{1}{2}, j}-u_{i-\frac{1}{2}, j+1}\right)}{\delta x}+\right. \\
& \left.\frac{v_{i+1, j+\frac{1}{2}}\left(u_{i+\frac{1}{2}, j}+u_{i+\frac{1}{2}, j+1}\right)-v_{i-1, j+\frac{1}{2}}\left(u_{i-\frac{1}{2}, j}+u_{i-\frac{1}{2}, j+1}\right)}{\delta x}\right]- \\
& \frac{1}{R e} \nu\left(q_{i, j+\frac{1}{2}}\right)\left[\left(\frac{u_{i+\frac{1}{2}, j+1}-u_{i+\frac{1}{2}, j}-u_{i-\frac{1}{2}, j+1}+u_{i-\frac{1}{2}, j}}{\delta x \delta y}\right)-\right. \\
& \left.\left(\frac{v_{i+1, j+\frac{1}{2}}-2 v_{i, j+\frac{1}{2}}+v_{i-1, j+\frac{1}{2}}}{(\delta x)^{2}}\right)\right]+\frac{1}{R e}\left[2\left(\frac{v_{i, j+\frac{3}{2}}-v_{i, j-\frac{1}{2}}}{2 \delta y}\right)\left[\frac{\partial \nu}{\partial y}\right]_{i, j+\frac{1}{2}}+\right. \\
& \left.\left[\left(\frac{u_{i+\frac{1}{2}, j+1}+u_{i-\frac{1}{2}, j+1}-u_{i+\frac{1}{2}, j}-u_{i-\frac{1}{2}, j}}{2 \delta y}\right)+\left(\frac{v_{i+1, j+\frac{1}{2}}-v_{i-1, j+\frac{1}{2}}}{2 \delta x}\right)\right]\left[\frac{\partial \nu}{\partial x}\right]_{i, j+\frac{1}{2}}\right]+ \\
& \frac{1}{F r^{2}} g_{y}
\end{aligned}
$$

onde $\left[\frac{\partial \nu}{\partial x}\right]_{i+\frac{1}{2}, j},\left[\frac{\partial \nu}{\partial y}\right]_{i+\frac{1}{2}, j},\left[\frac{\partial \nu}{\partial x}\right]_{i, j+\frac{1}{2}} \mathrm{e}\left[\frac{\partial \nu}{\partial y}\right]_{i, j+\frac{1}{2}}$ foram obtidos anteriormente.

\subsection{Condições de Contorno na Superfície Livre'}

As condições de contorno para uma superfície livre bidimensional são dadas pelas equações 1.38 e 1.39, as quais são aproximadas por diferenças finitas locais.

De acordo com a figura 3.3 [14], aproximam-se as equações 2.8 e 2.9 :

$$
\begin{gathered}
p_{i, j}=\frac{2 \nu\left(q_{i, j}\right)}{R e}\left(\frac{u_{i+\frac{1}{2}, j}-u_{i-\frac{1}{2}, j}}{\delta x}\right) \\
\left(\frac{u_{i+\frac{1}{2}, j+1}-u_{i+\frac{1}{2}, j}}{\delta y}\right)+\left(\frac{v_{i+1, j+\frac{1}{2}}-v_{i, j+\frac{1}{2}}}{\delta x}\right)=0 .
\end{gathered}
$$




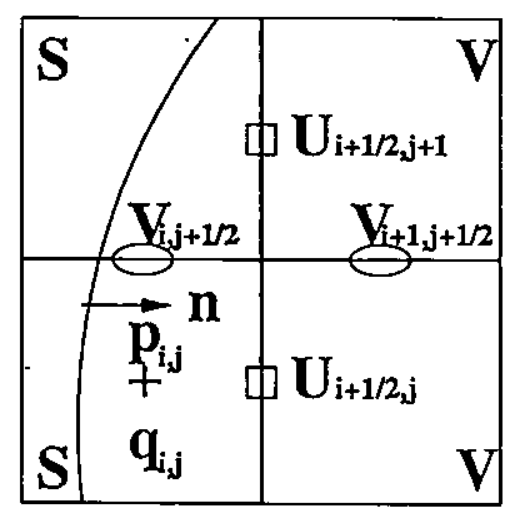

Figura 3.3: Discretização da célula (S) com o lado direito em contato com uma célula (V)

Utilizando-se 2.9 e a equação da continuidade, a razão de cisalhamento aproximada no ponto $(i, j)$ torna-se:

$$
q_{i, j}=2\left|\frac{u_{i+\frac{1}{2}, j}-u_{i-\frac{1}{2}, j}}{\delta x}\right|
$$

Considerando-se a figura 3.4 [14], as equações 2.10 e 2.11 são aproximadas:

$$
\begin{aligned}
p_{i, j}= & \frac{\nu\left(q_{i, j}\right)}{2 R e}\left[\left(\frac{u_{i+\frac{1}{2}, j}+u_{i-\frac{1}{2}, j}-u_{i+\frac{1}{2}, j-1}-u_{i-\frac{1}{2}, j-1}}{\delta y}\right)\right. \\
& \left.+\left(\frac{v_{i, j+\frac{1}{2}}+v_{i, j-\frac{1}{2}}-v_{i-1, j+\frac{1}{2}}-v_{i-1, j-\frac{1}{2}}}{\delta x}\right)\right] \\
& \left(\frac{u_{i+\frac{1}{2}, j}-u_{i-\frac{1}{2}, j}}{\delta x}\right)-\left(\frac{v_{i, j+\frac{1}{2}}-v_{i, j-\frac{1}{2}}}{\delta y}\right)=0 .
\end{aligned}
$$

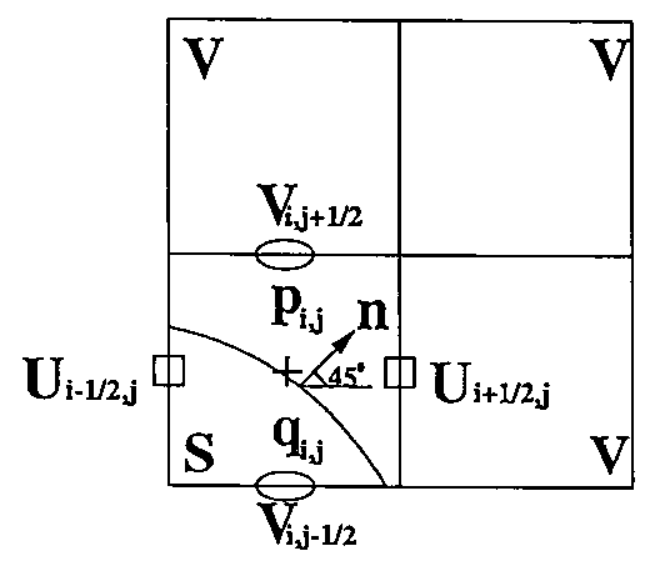

Figura 3.4: Discretização da célula (S) com dois lados em contato com células (V) 
A aproximação da razão de cisalhamento no centro da célula é dada por:

$$
\begin{gathered}
q_{i, j}=\frac{1}{2} \mid\left(\frac{u_{i+\frac{1}{2}, j}+u_{i-\frac{1}{2}, j}-u_{i+\frac{1}{2}, j-1}-u_{i-\frac{1}{2}, j-1}}{\delta y}\right) \\
+\left(\frac{v_{i, j+\frac{1}{2}}+v_{i, j-\frac{1}{2}}-v_{i-1, j+\frac{1}{2}}-v_{i-1, j-\frac{1}{2}}}{\delta x}\right) \mid .
\end{gathered}
$$

\subsection{Condições de Contorno na Superfície Rígida}

O método utilizado para o cálculo das velocidades $u$ e $v$ nas faces das células de fronteira é a interpolação linear. Essas células podem ter um ou dois lados adjacentes as células de superfície ou as células cheias.

Tratando-se de problemas bidimensionais têm-se oito casos de condiçōes de contorno rígido e, para problemas tridimensionais têm-se 26 casos.

De modo específico, as células $\mathrm{F}$ que têm uma face em contato com células $\mathrm{S}$ ou $\mathrm{C}$ apresentam quatro possíveis casos:

- células $\mathrm{F}$ com a face $i+\frac{1}{2}$ em contato com células $\mathrm{S}$ ou ${ }^{-} \mathrm{C}$;

- células F com a face $i-\frac{1}{2}$ em contato com células $S$ ou $C$;

- células F com a face $j+\frac{1}{2}$ em contato com células $\mathrm{S}$ ou $\mathrm{C}$;

- células F com a face $j-\frac{1}{2}$ em contato com células $\mathrm{S}$ ou $\mathrm{C}$.

$\mathrm{E}$ os quatro casos possíveis de células $\mathrm{F}$ que têm as duas faces em contato com células $\mathrm{S}$ ou $\mathrm{C}$ são:

- células F com as faces $i+\frac{1}{2}$ e $j+\frac{1}{2}$ em contato com células $\mathrm{S}$ ou $\mathrm{C}$;

- células $\mathrm{F}$ com as faces $i-\frac{1}{2}$ e $j+\frac{1}{2}$ em contato com células $\mathrm{S}$ ou $\mathrm{C}$;

- células $\mathrm{F}$ com as faces $i+\frac{1}{2}$ e $j-\frac{1}{2}$ em contato com células $\mathrm{S}$ ou $\mathrm{C}$;

- células F com as faces $i-\frac{1}{2}$ e $j-\frac{1}{2}$ em contato com células $\mathrm{S}$ ou $\mathrm{C}$.

Um primeiro exemplo de aplicação do método pode ser considerado conforme a figura 3.5. Trata-se do caso em que uma célula $\mathrm{F}$ tem a face $i+\frac{1}{2}$ em contato com uma célula $\mathrm{C}$ ou $\mathrm{S}$. 


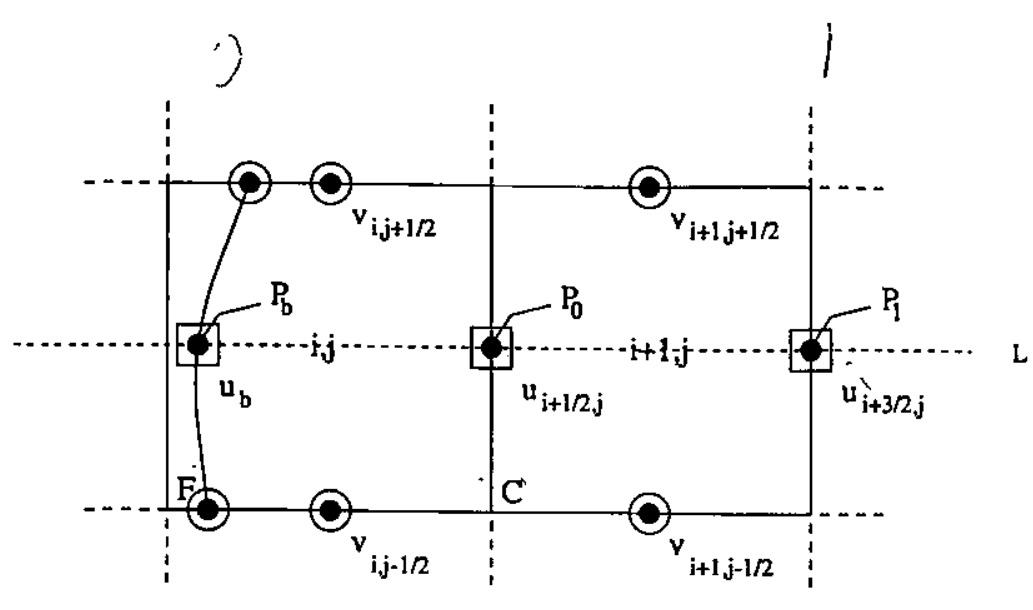

Figura 3.5: Célula F com a face $i+\frac{1}{2}$ em contato com células $\mathrm{S}$ ou $\mathrm{C}$

Para calcular as velocidades $\tilde{u}$ e $\tilde{v}$ a partir das equaçoes de momento discretizadas 3.1 e 3.2 , é necessário obter as velocidades $u_{i+\frac{1}{2}, j}, v_{i, j+\frac{1}{2}}$ e $v_{i, j-\frac{1}{2}}$, por meio da interpolação linear.

Considera-se $P_{0}=\left(x_{i+\frac{1}{2}}, y_{j}\right), \overline{P_{1}}=\left(x_{i+\frac{3}{2}}, y_{j}\right)$ e $P_{b}=\left(x_{u_{b}}, y_{j}\right)$, onde $x_{u_{b}}$ representa a intersecção entre a linha definida por $P_{0}$ e $P_{1}$ com a superfície da fronteira. Calcula-se $u_{i+\frac{1}{2}, j}$ fazendo uma interpolação linear entre $P_{b}$ e $P_{1}$ :

$$
u_{i+\frac{1}{2}, j}=\frac{x_{i+\frac{1}{2}}-x_{u_{b}}}{x_{i+\frac{3}{2}}-x_{u_{b}}} \cdot u_{i+\frac{3}{2}, j}+\frac{\delta x}{x_{u_{b}}-x_{i+\frac{3}{2}}} \cdot u_{b}
$$

em que $\delta x=x_{i+\frac{3}{2}}-x_{i+\frac{1}{2}}$.

Obtém-se o cálculo para a velocidade $v$ por analogia ao cálculo de $u$.

No caso de células $\mathrm{F}$ com duas faces em contato com células $\mathrm{S}$ ou $\mathrm{C}$ podem ocorrer duas possibilidades para se efetuar a interpolação como, por exemplo, mostra a figura 3.6. 


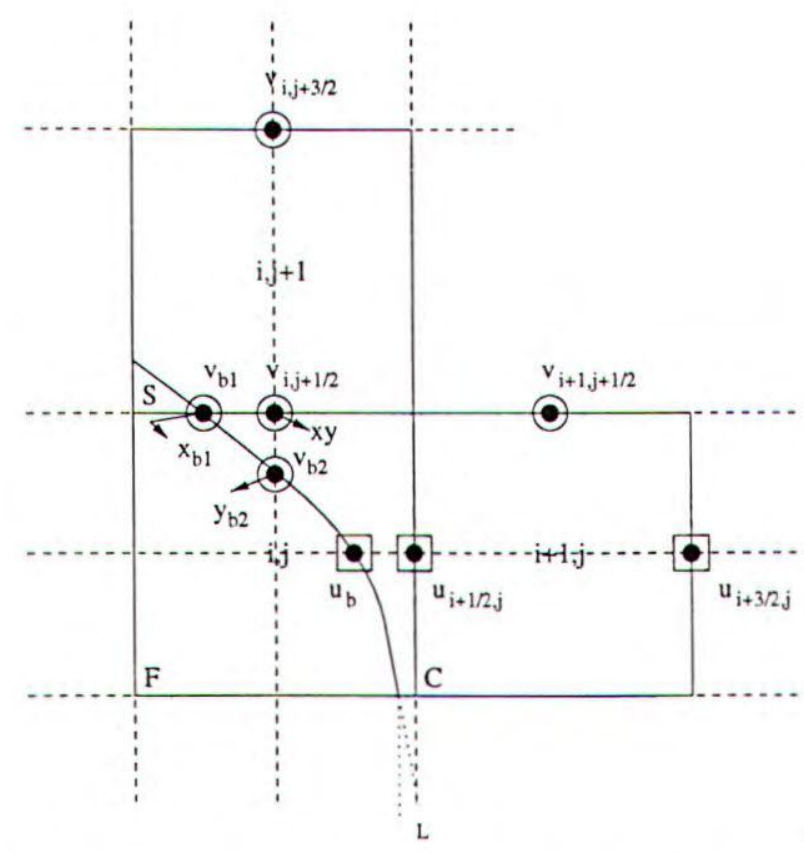

... Linhas de interseç̧ões

Figura 3.6: Célula $\mathrm{F}$ com a face $i+\frac{1}{2}$ e $j+\frac{1}{2}$ em contato com células $\mathrm{S}$ ou $\mathrm{C}$

A interpolação para a velocidade $v$ poderá ser feita na direção $x$ ou na direção $y$. Assim, deve-se escolher a direção onde $\left|x_{b_{1}}-x_{y}\right|$ ou $\left|y_{b_{2}}-x_{y}\right|$ for menor.

Determinada a direção, calcula-se $v$ seguindo os cálculos feitos anteriormente.

Para o cálculo de $u$ se a superfície da fronteira não intersepta a linha $L$, tem-se apenas uma opção para efetuar a interpolação, e a resolução segue de forma análoga ao primeiro exemplo. Caso contrário, o cálculo de $u$ torna-se análogo ao segundo exemplo.

Os demais casos de condições de contorno rígido decorrem de forma análoga a estes dois exemplos.

\subsection{A Discretização da Equação de Poisson}

A equação

$$
\nabla^{2} \psi(x, t)=\nabla \cdot \tilde{u}(x, t),
$$

que aplicada sobre uma malha diferencial, fornece:

$$
\left.\nabla^{2} \psi\right|_{i, j}=\tilde{D}_{i, j}
$$

onde

$$
\tilde{D}_{i, j}=\left(\frac{\partial \tilde{u}}{\partial x}+\frac{\partial \tilde{v}}{\partial y}\right)_{i, j}=\nabla \cdot \tilde{u}(x, t),
$$


ou seja

$$
\tilde{D}_{i, j}=\left(\frac{\partial^{2} \psi}{\partial x^{2}}+\frac{\partial^{2} \psi}{\partial y^{2}}\right)_{i, j}
$$

Utilizando a expansão em Série de Taylor para desenvolver o termo $\frac{\partial^{2} \psi}{\partial x^{2}}\left(x_{i}, y_{j}\right)$, por aproximações progressiva e regressiva, obtém-se:

$$
\begin{aligned}
\psi\left(x_{i}+\Delta x, y_{j}\right)=\psi\left(x_{i}, y_{j}\right)+ & \frac{\partial \psi}{\partial x}\left(x_{i}, y_{j}\right) \Delta x+\frac{1}{2} \frac{\partial^{2} \psi}{\partial x^{2}}\left(x_{i}, y_{j}\right) \Delta x^{2}+\frac{1}{6} \frac{\partial^{3} \psi}{\partial x^{3}}\left(x_{i}, y_{j}\right) \Delta x^{3} \\
& +\frac{1}{24} \frac{\partial^{4} \psi}{\partial x^{4}}\left(x_{i}, y_{j}\right) \Delta x^{4}+\ldots \\
\psi\left(x_{i}-\Delta x, y_{j}\right)=\psi\left(x_{i}, y_{j}\right)- & \frac{\partial \psi}{\partial x}\left(x_{i}, y_{j}\right) \Delta x+\frac{1}{2} \frac{\partial^{2} \psi}{\partial x^{2}}\left(x_{i}, y_{j}\right) \Delta x^{2}-\frac{1}{6} \frac{\partial^{3} \psi}{\partial x^{3}}\left(x_{i}, y_{j}\right) \Delta x^{3} \\
& +\frac{1}{24} \frac{\partial^{4} \psi}{\partial x^{4}}\left(x_{i}, y_{j}\right) \Delta x^{4}-\ldots
\end{aligned}
$$

Adicionando 3.4 e 3.5 , tem-se:

$$
\psi\left(x_{i}+\Delta x, y_{j}\right)+\psi\left(x_{i}-\Delta x, y_{j}\right)=2 \psi\left(x_{i}, y_{j}\right)+\frac{\partial^{2} \psi}{\partial x^{2}}\left(x_{i}, y_{j}\right) \Delta x^{2}+O\left(\Delta x^{4}\right)
$$

E assim,

$$
\frac{\partial^{2} \psi}{\partial x^{2}}\left(x_{i}, y_{j}\right)=\frac{\psi\left(x_{i}+\Delta x, y_{j}\right)-2 \psi\left(x_{i}, y_{j}\right)+\psi\left(x_{i}-\Delta x, y_{j}\right)-O\left(\Delta x^{4}\right)}{\Delta x^{2}} .
$$

De forma análoga, obtém-se:

$$
\frac{\partial^{2} \psi}{\partial y^{2}}\left(x_{i}, y_{j}\right)=\frac{\psi\left(x_{i}, y_{j}+\Delta y\right)-2 \psi\left(x_{i}, y_{j}\right)+\psi\left(x_{i}, y_{j}-\Delta y\right)-O\left(\Delta y^{4}\right)}{\Delta y^{2}} .
$$


Considerando $h=\Delta x=\Delta y=\delta x=\delta y$, substituindo 3.6 e 3.7 na equação de Poisson 3.3, obtém-se

$$
4 \psi_{i, j}-\psi_{i+1, j}-\psi_{i-1, j}-\psi_{i, j+1}-\psi_{i, j-1}=-h^{2} \tilde{D}_{i, j}
$$

com erro de truncamento de $O\left(h^{2}\right)$ onde $\tilde{D}_{i, j}$ é dado por

$$
\tilde{D}_{i, j}=\left(\frac{\partial \tilde{u}}{\partial x}+\frac{\partial \tilde{v}}{\partial y}\right)_{i, j}
$$

que aproximado torna-se:

$$
\tilde{D}_{i, j}=\left(\frac{\tilde{u}_{i+\frac{1}{2}, j}-\tilde{u}_{i-\frac{1}{2}, j}}{\delta x}\right)+\left(\frac{\tilde{v}_{i, j+\frac{1}{2}}-\tilde{v}_{i, j-\frac{1}{2}}}{\delta y}\right) .
$$

\subsection{A Solução da Equação de Poisson}

A equação de Poisson discretizada 3.8 conduz a um sistema linear representado por

$$
A X=D,
$$

onde $A$ é uma matriz simétrica esparsa e definida positiva de ordem $n$, com $n$ representando o número de células cheias $(\mathrm{C})$ dentro da malha.

Para resolver este sistema é empregado o Método dos Gradientes Conjugados. A solução para a pressão, obtida no passo anterior, é considerada uma boa aproximação para o passo corrente e o método utilizado busca a solução na direção dos gradientes. Com isso, a cada ciclo de cálculo, ocorre um progresso uniforme na direção da solução em cada passo e portanto, o método converge rapidamente.

Aplicando-se a equação 3.8 em cada célula $\mathrm{C}$ da malha, é montada a matriz $\mathrm{A}$. Cada célula $\mathrm{C}$ representa uma linha dos coeficientes de $\mathrm{A}$ e um elemento em $\mathrm{D}$ (o vetor divergente de ordem n). A condição homogênea de Neumann aplicada sobre uma célula $\mathrm{C}$, que tem os lados adjacentes com a fronteira virtual, modifica os termos da diagonal principal. A condição homogênea de Dirichlet sobre uma célula $\mathrm{C}$ que faz fronteira com células de superfície, zeram termos fora da diagonal.

Depois que a matriz A é montada, observa-se que esta contém uma quantidade relativamente baixa de elementos não nulos. Em tais circunstâncias é importante poder resolver o sistema sem modificar a matriz, destruindo ou reduzindo sua esparsidade. Os 
processos iterativos são muito eficientes neste sentido, já que os métodos diretos provocam alterações destes elementos (fill-in).

Aplicando-sc o Método dos Gradientes Conjugados, X é inicializado como um vetor da função potencial no passo anterior. Conforme a disposição dos elementos da matriz A, pode-se empregar um precondicionador para acelerar a convergência do método.

\subsection{Controle do Passo no Tempo}

O tamanho do passo dado no tempo em cada ciclo de cálculo pode ser reduzido fazendo uso de uma rotina adaptativa [3]. Assim, em cada ciclo, poderá automaticamente ser escolhido o passo a ser dado no tempo mais apropriado para o campo de velocidade. Para isto, são exigidas duas restrições de estabilidade:

i) Dado um intervalo de tempo, nenhuma partícula poderá atravessar mais de uma célula, isto é,

$$
|u| \delta t<\delta x \quad \text { e } \quad|v| \delta t<\delta y
$$

onde $\delta x$ e $\delta y$ são os espaçamentos da malha e $\delta t$ é o passo dado no tempo.

Condição suficiente para satisfazer a restrição anterior:

$$
\left|U_{\max }\right| \delta t<\delta x \quad \text { e } \quad\left|V_{\max }\right| \delta t<\delta y,
$$

em que $U_{\max }$ e $V_{\max }$ são os valores máximos de $u$ e $v$, respectivamente;

ii) Conforme a discretização explícita das equações de momento, a segunda restrição de estabilidade envolve a viscosidade e o número de Reynolds:

$$
2 \nu(q) \delta t<\operatorname{Re} \frac{\delta x^{2} \delta y^{2}}{\delta x^{2}+\delta y^{2}} .
$$

Condição suficiente para que a restrição acima seja satisfeita:

$$
2 \nu_{\max } \delta t<R e \frac{\delta x^{2} \delta y^{2}}{\delta x^{2}+\delta y^{2}},
$$

onde $\nu_{\max }=\max \left|\nu_{i, j}\right|$. 


\subsection{O Movimento das Partículas}

A representação do fluido é feita através de partículas virtuais, as quais permitem indicar a posição da superfície livre, a fim de que possam ser determinadas as configuraçōes das células de Superfície (S).

No final de cada passo dado no tempo, as partículas são atualizadas e assim, obtém-se a dinâmica do movimento do fluido.

Utilizando o Método de Euler resolve-se

$$
\frac{d x}{d t}=u \quad \text { e } \quad \frac{d y}{d t}=v
$$

e obtém-se a nova coordenada de representação do fluido. Assim, apọ́s a atualização da velocidade, o movimento das partículas ocorre de acordo com

$$
\begin{aligned}
& x_{p}^{n+1}=x_{p}^{n}+u_{p} \delta t^{n+1} \\
& y_{p}^{n+1}=y_{p}^{n}+v_{p} \delta t^{n+1}
\end{aligned}
$$

onde $\left(x_{p}^{n}, y_{p}^{n}\right)$ representa a posição corrente da partícula, $\delta t^{n+1}$ é o passo no tempo atuaı e $\left(x_{p}^{n+1}, y_{p}^{n+1}\right)$ é a posição atualizada. As velocidades $u_{p}, v_{p}$ são calculadas usando uma interpolação bilinear, envolvendo as quatro velocidades $u$ e $v$ mais próximas. 


\section{Capítulo 4}

\section{O Ambiente Freeflow-3D}

\subsection{Considerações Iniciais}

Este capítulo descreve o sistema Freeflow-3D [3], um ambiente de modelagem, simulação e visualização de escoamentos tridimensionais de fluidos incompressiveis com superfícies livres. Este ambiente é composto por um modelador - Modflow-3D, que facilita a entrada de dados, um simulador - Simflow-3D, que implementa as equaçōes governantes e condiçōes de fronteira, um visualizador - Visflow-3D, que exibe imagens bidimensionais e tridimensionais do escoamento e um reiniciador - Resimflow-3D, o qual permite reiniciar uma simulação com a alteração de alguns parâmetros.

- Os módulos do sistema comunicam-se através de arquivos de dados e os objetos geométricos (fluidos, contêineres, injetores e ejetores) são representados por uma estrutura de dados B-Rep (Boundary Representation).

\subsection{Freeflow-3D}

$\mathrm{O}$ ambiente de simulação tridimensional para escoamentos incompressíveis com superfícies livres é composto por quatro módulos distintos:

- Modflow-3D (modelador de moldes e escoamentos): trata-se de um módulo interativo para a especificação de um modelo de escoamento de fluido. Este módulo inclui a definição de elementos no domínio do escoamento, como contêineres, injetores, fluidos e propriedades do escoamento. Técnicas de modelagem de sólidos são utilizadas para criar os objetos geométricos; 
- Simflow-3D (simulador de escoamentos): este módulo é a parte central do Freeflow-3D, pois implementa a discretização das equações governantes e condiçōes de contorno. Este sistema emprega o método GENSMAC-3D [24], o qual é uma variante do método GENSMAC, com partículas virtuais apenas na superfície livre do fluido;

- Visflow-3D (visualizador de escoamentos): este módulo interativo permite ao usuário visualizar os resultados produzidos pelo módulo Simflow-3D, utilizando técnicas de visualização;

- Resimflow-3D (reiniciador de escoamentos): trata-se de um sistema interativo que permite alterar alguns parâmetros do modelo de escoamento.

Os quatro módulos foram implementados em linguagem $\mathrm{C}$ sobre o sistema operacional UNIX. As interfaces gráficas para o modelador, o visualizador e o reiniciador utilizam o sistema de janelas Xview sobre o X-Windows. A estrutura de dados foi designada para facilitar o acesso às informações e possui independência de dados, a fim de facilitar a manutenção e extensōes do sistema. A comunicação entre os módulos do sistema é efetuada através de arquivos de dados.

\subsection{Estrutura de Dados}

O Freeflow-3D possui duas classes de dados: diretos e indiretos. O conjunto dos dados diretos contém dados referentes ao domínio, velocidade, pressão, células, parâmetros usados pelo simulador e a representação dos objetos geométricos do modelo. Este conjunto é subdividido em dados estáticos e dinâmicos.

Os dados estáticos não são modificados durante a simulação e compreendem a definição do domínio, a discretização, parâmetros de adimensionalização e algumas propriedades do escoamento e do fluido tais como a viscosidade e o campo de gravidade, entre outros. Os dados dinâmicos são os que se modificam durante a simulação.e englobam a velocidade e pressão (representados por matrizes), a configuração do conjjunto de células e a representaçāo dos objetos geométricos.

Todas as células sāo representadas por matrizes e cada grupo de células I, F, S, C e $\mathrm{E}$ é representado por uma estrutura de dados do tipo árvore, contendo informaçōes e configurações destas células. 
Os objetos geométricos têm suas fronteiras aproximadas pór uma superfície linear por partes e são representados pela estrutura de dados "half-edge", a qual é um tipo de estrutura B-Rep (Boundary Representation) [13], utilizada para representar faces, arestas, vértices e suas relações topológicas. Esta estrutura foi originalmente desenvolvida para representar por sua fronteira sólidos rígidos com uma única componente de fronteira, sendo que a geometria das faces e arestas pode ser não linear.

A inserção e a eliminação de dados na estrutura "half-edge" são feitas através de operadores de Euler, os quais garantem a consistência topológica do objeto representado. Entre outros tipos de representação de sólidos, a representação B-Rep foi escolhida devido a sua concisão e eficiência para representar as modificaçōes locais na fronteira dos objetos.

A estrutura de dados empregada para representar a classe de dados indiretos foi constituída com base em três aspectos principais: a representação dos objetos geométricos por sua fronteira, o desempenho dos algoritmos envolvidơs no simulador e a minimização da interdependência dos dados.

Os dados indiretos consistem em três estruturas [16]:

- Containers: representam os contêineres e é composta de dados geométricos (B-Rep), tipos de condições de contorno, informações armazenadas em árvore sobre as células que definem esse contêiner $\dot{e}$ atributos específicos do contêiner representado;

- Inflows: representam os injetores e é composta de dados geométricos (B-Rep), características do injetor, informações sobre os contêineres e o fluido relacionado a esse injetor, uma árvore que armazena informações sobre as células que o definem e atributos específicos;

- Outflows: representam os ejetores e é composta de dados geométricos (B-Rep), tipos de condições de contorno, informações sobre o contêiner que o contém, uma árvore que armazena informações sobre as células que definem esse ejetor e atributos específicos.

Essa estrutura de dados é utilizada pelo modelador que possibilita a construção da configuração inicial do escoamento, utilizada no simulador, e foi desenvolvida a fim de possibilitar futuras extensões, como por exemplo, contêineres de formas arbitrárias, escoamentos nāo-Newtonianos e contêineres em movimento. 


\subsection{O Modelador}

O módulo Modflow-3D é responsável pela inicialização de dados para a simulação. Este módulo consiste de uma interface gráfica amigável para a introdução de dados relacionados ao escoamento, como o domínio, a malha, as células, valores de referência para a velocidade e comprimento, viscosidade, tipo de escoamento, parâmetros e tolerâncias usadas pelo simulador e os objetos geométricos que compõem o modelo. O modelador também permite a visualizaçà̃o dos dados geométricos gerados. Os objetos são visualizados através de técnicas de "rendering" (iluminaçã̀), as quais facilitam a interpretação dos dados pelo usuário. Além disso, o usuário pode dispor de operações geométricas usuais, tais como rotação, translação e ampliação, e também escolher o tipo de projeção (paralela, perspectiva). Outro recurso muito útil é a possibilidade de alterar parâmetros como a distância do observador, o tipo de "rendering" (Wire-Frame, Flat, Gourand, Phong), potência da luz ambiente, luz direcional e propriedades de visualização dos objetos (cor, visibilidade, transparência e propriedades de reflexão da luz).

Os objetos geométricos são representados pela estrutura "half-edge" de dados BRep. Esta representação é efetuada por uma técnica conhecida de modelagem: instanciamento de primitivas tridimensionais. Partindo-se de objetos pré-definidos, esta técnica permite a escolha de objetos novos simplesmente pela definição de seus parâmetros.

Os objetos pré-definidos atualmente são:

- Caixa retangular aberta - definida por um ponto de referência, comprimento, largura, altura, espessura e inclinação nas direções $x$ e $y$;

- Caixa cilíndrica com fundo chato - definida por um ponto de referência, raio, altura, espessura, inclinação e resolução;

- Caixa cilíndrica com fundo esférico - definida por um ponto de referência (centro), raio, altura, espessura e resolução;

- Caixa poliedral com fundo chato - definida por um ponto de referência, raio, altura, espessura, inclinação e resolução;

- Caixa poliedral com fundo esférico - definida pelo centro, raio, altura, espessura e resolução;

- Paralelepípedo - definido por um ponto de referência, altura, largura e comprimento;

- Toro retangular - definido por um ponto de referência, comprimento, altura, largura e 
espessura;

- Esfera - definido pelo centro, raio e resoluçāo;

- Cilindro - definido pelo centro, raio, altura e resolução.

Todos estes objetos são utilizados na representação de contêineres. O paralelepípedo, o toro e o cilindro são utilizados para representar injetores e fluidos e a esfera é utilizada também na representação do fluido.

- Os dados restantes das estruturas Contêineres, Injetores e Ejetores são obtidos. diretamente do menu "pull-down". Por exemplo, a estrutura do Injetor também contém informações sobre: o plano e a orientação, velocidade, tipo de condição de fronteira, parâmetros utilizados para a inserção de partículas (número de partículas em cada direção e em cada célula), uma árvore armazenando o tipo de célula que define a discretização do injetor e seus atributos específicos (parâmetros de visualização e dados relacionados a definição é movimento do injetor).

As intersecções das linhas paralelas aos eixos principais com os objetos (contêineres e injetores), como mostra as figuras 3.5 e 3.6 do capítulo 3, são exigidas pelo simulador, em adição ao conjunto de células. Estas intersecções são calculadas a partir das faces da estrutura "half-edge" e armazenadas numa estrutura do tipo árvore, como já foi descrita anteriormente.

A figura 4.1 [3] ilustra a intersecção de uma face do objeto com uma célula, e a porção da célula no interior do objeto.

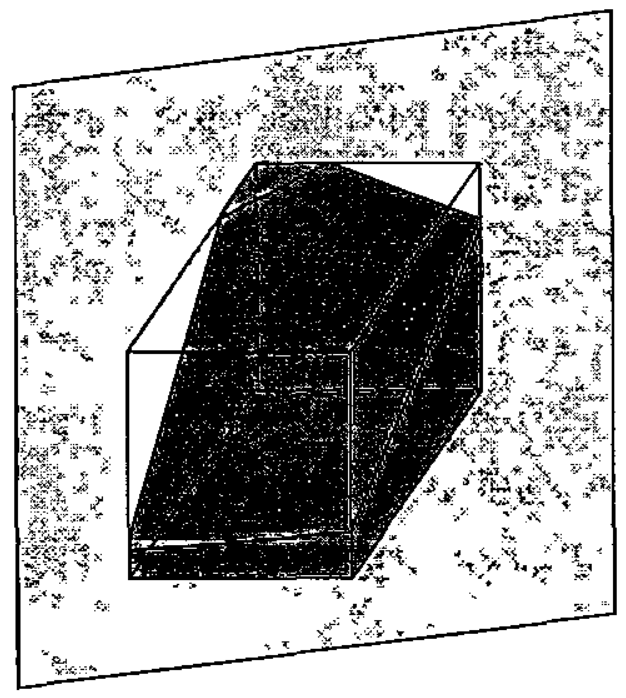

Figura 4.1: Face, células e intersecções. 
Em três dimensões são possíveis 27 intersecções, resultando do fato de serem 9 retas paralelas aos eixos principais. Essas intersecções serão determinadas no caso de células F, I e E como consta no capítulo 3, seção 3.7, para o caso bidimensional.

\subsection{O Simulador}

A partir do método GENSMAC desenvolveu-se o método GENSMAC-3D [24], que é empregado no módulo Simflow-3D. Este método aproxima a superfície livre por uma superfície linear por partes representada pela estrutura "half-edge", que favorece as modificações locais mantendo o controle global da topologia.

As equações de Navier-Stokes juntamente com a equação da continuidade são as equações que regem os escoamentos transientes com superfícies livres. A discretização do domínio (definido pelo paralelepípedo que contém os componentes do escoamento) é feita uniformemente em cada direção no método GENSMAC-3D, construindo uma malha onde todas as células possuem as mesmas dimensões $(d x, d y, d z)$. Desse modo, classificase essas células a todo instante de tempo, de acordo com o fluido e os contornos rígidos (contêineres, injetores e ejetores), como mostra o capítulo 2 [3].

A figura 4.2 [3] mostra o posicionamento das variáveis:

$\diamond$ variável pressão: no centro da célula;

$\diamond$ variáveis $u, v, w$ : acompanha as direções $x, y, z$ da velocidade $\mathbf{u}$, posicionando-se nas faces frontal, lateral direita e superior da célula.

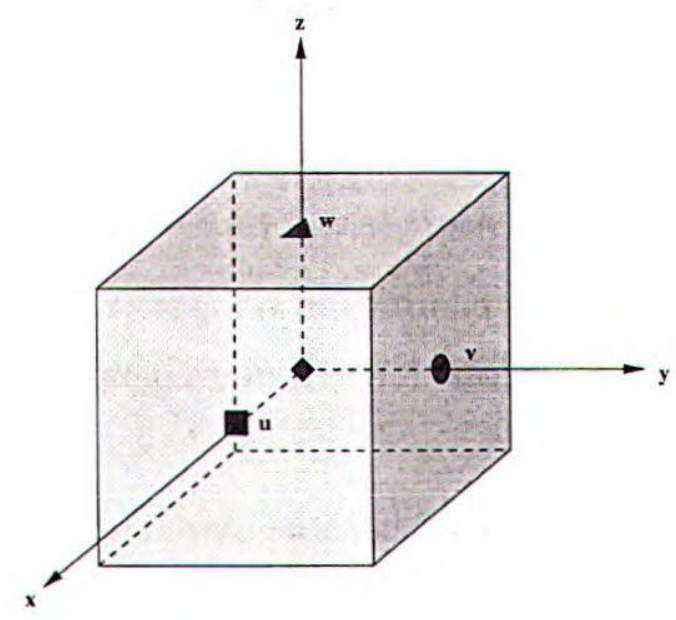

Figura 4.2: Célula, discretização da pressão e velocidade 
O método GENSMAC-3D utiliza a pressão e velocidade discretizadas na malha diferenciada.

Os passos a seguir são análogos aos estudados no capítulo 2, seção 2.3 .

As condições de contorno, na superfície livre, para a pressão e velocidade são dadas pelas relações :

$$
(T \cdot \mathbf{n}) \cdot \mathbf{n}=0, \quad(T \cdot \mathbf{n}) \cdot \mathrm{m}_{1}=0 \quad \text { e } \quad(T \cdot \mathbf{n}) \cdot \mathbf{m}_{2}=0,
$$

onde:

$\diamond \mathrm{n}=\left(n_{1}, n_{2}, n_{3}\right)$ é o vetor normal unitário;

$\diamond T$ é o tensor de estresses;

$\diamond \mathrm{m}_{1}$ e $\mathrm{m}_{2}$ são vetores tangentes à superfície livre.

As condições de não escorregamento e "prescribed inflow" (injeção) compõem as condições de contorno na superfície rígida. Essas condições de contorno são aplicadas na fronteira delimitada pelo contorno rígido (contêineres e injetores) em contato com o fluido.

A estabilidade do processo numérico depende de como as condições na fronteira livre são aproximadas, fazendo-se uma analogia ao problema bidimensional. Assim, tentase uma aproximação para essas condições, tendo pois que a célula seja suficientemente pequena para que a superfície possa ser representada localmente por uma superfície planar.

Ao final, a posição das partículas marcadoras são atualizadas em três etapas:

- mover a superfície: análogo ao capítulo 2, seção 2.3 (vi);

- inserir partículas (representadas pelos vértices da estrutura "half-edge"):

॰ Divide-se cada face que representa a superfície livre em faces menores sempre que sua área seja superior a $(0.5 * \min \{d x, d y, d z\})^{2}$;

$\diamond$ Dividi-se cada aresta com comprimento superior a $0.75 * \min \{d x, d y, d z\}$. Assim cada célula que intercepta a superfície livre contém pelo menos um vértice que representa uma partícula.

Para o bom funcionamento do sistema o processo de inserção é um dado importante, pois de acordo com o movimento do fluido, as partículas que representam a superfície podem ficar distantes umas das outras e assim perdem-se os detalhes da representação. Pode ocorrer que uma célula da superfície não contenha partículas devido a definição das células ter sido feita no instante de tempo anterior, podendo haver confusão no algoritmo 
de definição das células no tempo atualizado.

- Eliminar partículas: se faz quando uma aresta contém vértices numa mesma célula e seu tamanho é inferior a $0.5 * \min \{d x, d y, d z\}$. Esse processo é importante para a diminuição de dados.

\subsection{O Visualizador}

O Visflow-3D é o módulo de visualização, responsável por apresentar os resultados do simulador.

Os resultados gerados pelo simulador são dados sobre o modelo de escoamento, a representação dos objetos geométricos e propriedades em instantes pré-definidos.

A entrada de dados do visualizador é feita por arquivos. Um arquivo armazena os dados que independem do tempo, e os dados que dependem do tempo são armazenados por uma série de arquivos, contendo informações sobre os objetos geométricos, propriedades, configurações das células, tempo e ciclo da simulação.

Para facilitar a interpretação dos dados, os objetos são apresentados utilizando-se técnicas de "rendering", as mesmas utilizadas no módulo Modflow-3D, para a visualização da geometria (contêineres, injetores, ejetores, fluidos). Pode-se visualizar os objetos por meio de cortes por planos paralelos aos planos principais. Para a visualização das propriedades dos fluidos (pressão e velocidade), utiliza-se técnicas de "rendering", considerando a propriedade de interesse como uma textura. Uma outra técnica utilizada para a visualização das propriedades é através de curvas de nível dos valores de interesse, em cortes por planos.

A velocidade é visualizada pelas suas três componentes:

$\rightarrow u$ na direção $x$;

$\rightarrow v$ na direção $y$;

$\rightarrow w$ na direção $z$.

Após a escolha de uma propriedade, as superfícies dos fluidos são coloridas de acordo com o valor assumido por ela em cada ponto. Para facilitar o entendimento da figura usa-se uma faixa relacionando o valor da propriedade e as cores utilizadas. Essa faixa é representada simultaneamente e também informa a variação total da propriedade visualizada. 
Utilizando-se interpolação linear sobre os valores da propriedade das oito células mais próximas, obtém-se o valor da propriedade em um ponto da superfície. A figura 4.3 [3] apresenta um ponto na célnla no qual se quer calcular a pressão, e os oito pontos mais próximos das células adjacentes nos quais são armazenados os valores da pressão.

O eixo perpendicular define os cortes, assim como a quantidade de cortes e valor inicial e final dos planos de corte.

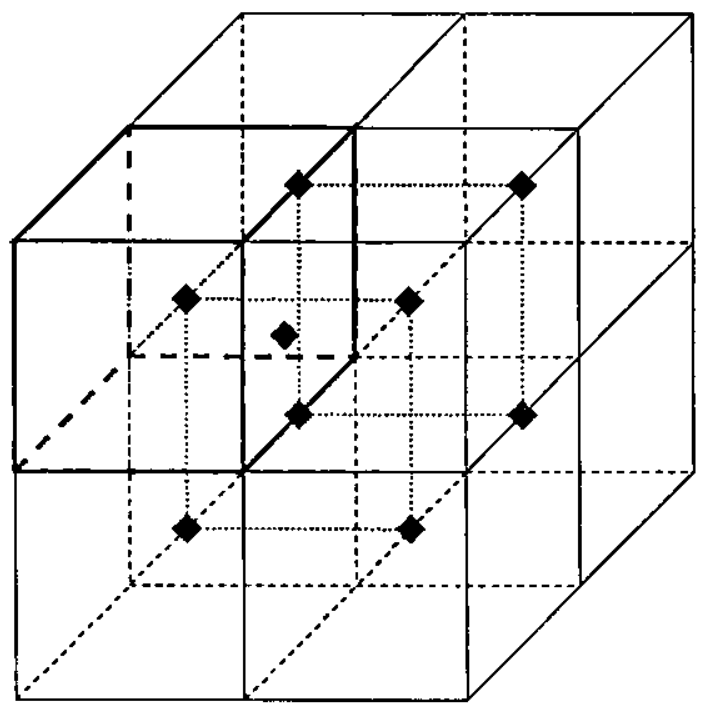

Figura 4.3: Células e intersecções com as retas

As propriedades são visualizadas através de cortes, sendo que a representação é feita com base nas curvas de níveis da propriedade.

\subsection{O Reiniciador}

O módulo Resimflow-3D é um sistema interativo capaz de reiniciar uma simulação em qualquer fase, e não apenas do seu início, ou então, efetuar modificações nos parâmetros do modelo de escoamento e prosseguir normalmente com as simulações após essas modificações serem implementadas. Essa propriedade se mostra de suma importância em problemas de percurso durante simulações longas em que, por exemplo, uma queda de energia da rede elétrica ou um problema técnico com os terminais que ocasiona uma parada na simulação.

Os dados do modelo de escoamento que podem ser alterados no reiniciador são:

- O ciclo e o tempo final; 
- Espaçamento de tempo para impressão e gravaçao automática;

- Tolerância para a equação de Poisson;

- Fatores de controle do passo.

\subsection{A Comunicação Entre os Módulos}

Os arquivos são os meios utilizados para a comunicação entre os módulos do sistema. Oito arquivos compõem os resultados gerados pelo modelador: quatro desses arquivos armazenam informações na estrutura "half-edge" de contêineres, injetores, ejetores e fluidos. Três arquivos armazenam informações nas estruturas indiretas de contêineres, injetores e ejetores e o oitavo arquivo armazena todos os dados iniciais, como: viscosidade, fatores e tamanho do passo dado no tempo, parâmetros escalares e propriedades do escoamento.

A estrutura desses oito arquivos de entrada do simulador e de saída do modelador é a mesma podendo assim, esses arquivos terem sido gerados tanto pelo modelador como pelo próprio simulador.

As funções dos quatro diferentes arquivos de saídas do simulador são:

- arquivos de saída normal: a simulação poderá ou não continuar a partir destes dađos;

- arquivos de saída forçada: para o caso de ocorrer algum erro durante a simulação;

- arquivos temporários salvos em tempos pré-definidos: para o caso de interrupção da simulação devido a algum problema do sistema ou da máquina;

- arquivos de visualização: para a visualização dos resultados.

A estrutura dos três primeiros arquivos é a mesma daqueles do modelador. $\mathrm{O}$ último, tem a mesma estrutura do visualizador.

Quatro arquivos contendo as informações sobre a estrutura "half-edge" de contêiṇeres, injetores, ejetores e fluidos criam a entrada de dados para o visualizador. Esses arquivos ão usados para visualizar os objetos:

Cinco arquivos adicionais contém os valores dos campos de velocidade e pressāo $u, v, p, w$ e as configurações das células, que são criados para visualizar as propriedades do escoamento. Um arquivo principal é criado, no início da simulação e utilizado em todo o decorrer do processo, contendo informaçōes sobre os dados estáticos.

Este sistema foi estruturado para permitir futuras extensões dos módulos, como 
por exemplo:

- Simflow-3D - extensão para os fluidos que apresentam tensão superficial, turbulência, e fluidos não-Newtonianos tais como: fluidos visco-elásticos, modelo "crossmodel" e modelo "power-law";

- Visflow-3D - efeitos de animação;

- Modflow-3D - considerar contêineres e injetores com formatos curvos;

- Modflow-3D - cada contêiner e injetor pode ter movimento independente durante a simulação. Este aspecto pode ser muito útil do ponto de vista prático na indústria alimentícia. Para isso, foi desenvolvido um modelador de movimentos como extensão do módulo Modflow-3D, o qual é apresentado no próximo capítulo. 


\section{Capítulo 5}

\section{O Modelador de Movimentos}

\subsection{Considerações Iniciais}

A primeira etapa deste projeto foi o desenvolvimento de um modelador de movimentos bidimensional, juntamente com sua interface gráfica, que está integrado ao sistema Freeflow-2D [9]. Em seguida, com os resultados obtidos a partir do modelador de movimentos em duas dimensões, desenvolveu-se o tridimensional, efetuando-se algumas adaptações.

Este capítulo dividi-se em duas partes. Na seção 5.2 descreve-se o modelador de movimentos bidimensional e na seção 5.3, o modelador de movimentos tridimensional. Em cada seção estão detalhadas as técnicas utilizadas para a construção do modelador de movimentos.

\subsection{O Modelador de Movimentos Bidimensional}

No modelador de moldes bidimensional é possivel a construção e manipulação de objetos através do instanciamento de primitivas, que fornece um conjunto de objetos geométricos básicos pré-definidos. Tanto o tamanho, como o formato, a posição e orientação são determinados por um conjunto de parâmetros especificados pelo usuário.

No modelador de movimentos definiu-se apenas movimentos rígidos, os quais englobam somente movimentos de translação e rotação em torno do centro de massa de cada objeto.

Dessa forma, a trajetória e a orientação desses objetos serão definidas a partir do 
centro de massa e do ângulo formado com relação ao eixo $x$,

$$
\mathrm{P}_{c}=\left(\begin{array}{c}
x_{c}(t) \\
y_{c}(t)
\end{array}\right) \quad \text { e } \quad \theta=f(t)
$$

em que $t$ é o tempo e as funções $x_{c}, y_{c}$ e $f$ são de classe $C^{2}$.

A figura 5.1 mostra um objeto pré-definido que poderá ser escolhido pelo usuário, onde $P_{c}$ e $P_{i}, i=1, . ., 4$, representam, respectivamente, o centro de massa e os vértices do objeto no instante inicial $t_{0}$.

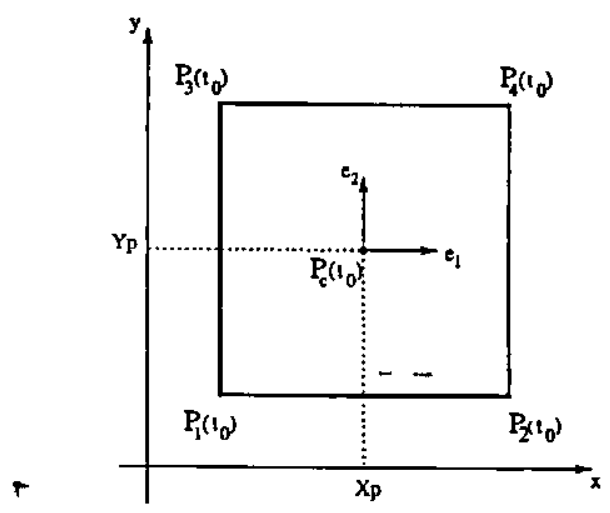

Figura 5.1: Objeto no instante $t_{0}$

Neste caso, o ângulo de orientação do objeto é igual a zero.

\subsubsection{Determinação do Centro de Massa}

No instante inicial, o centro de massa dos objetos será calculado a partir de seus vértices. Sob o ponto de vista geométrico, o centro de massa representa o baricentro dos objetos, calculado através de

$$
\mathrm{P}_{c}=\frac{1}{n} \sum_{i=1}^{n} P_{i}
$$

- ou seja $a_{2}$ uma média aritmética de todos os vértices do objeto onde

$$
\mathbf{P}_{c}=\left(\begin{array}{c}
x_{c} \\
y_{c}
\end{array}\right) \quad \text { e } \quad \mathbf{P}_{i}=\left(\begin{array}{c}
x_{i} \\
y_{i}
\end{array}\right)
$$


Durante a simulação, o centro de massa $\mathrm{P}_{c}$ e o ângulo de cada objeto pode estar previamente definido no modelador ou então ser calculado através de alguma técnica de aproximação, tal como, interpolação por splines cúbica.

\subsubsection{Definição do Movimento de Translação e Rotação a partir do Centro de Massa}

De acordo com o tempo, os objetos assumem uma novā posiç̧ão no decorrer da simulação. Para se obter a posição atual do objeto, primeiro é necessário determinar as coordenadas do centro de massa e ângulo de orientação, conforme o tipo de movimento especificado. Com isso, pode-se calcular a posição atualizada de seus vértices:

$$
\mathrm{P}_{i}(t)=\left(\begin{array}{c}
x_{i}(t) \\
y_{i}(t)
\end{array}\right)
$$

Considerando-se um objeto que se desloca conforme mostra a figura 5.2 .
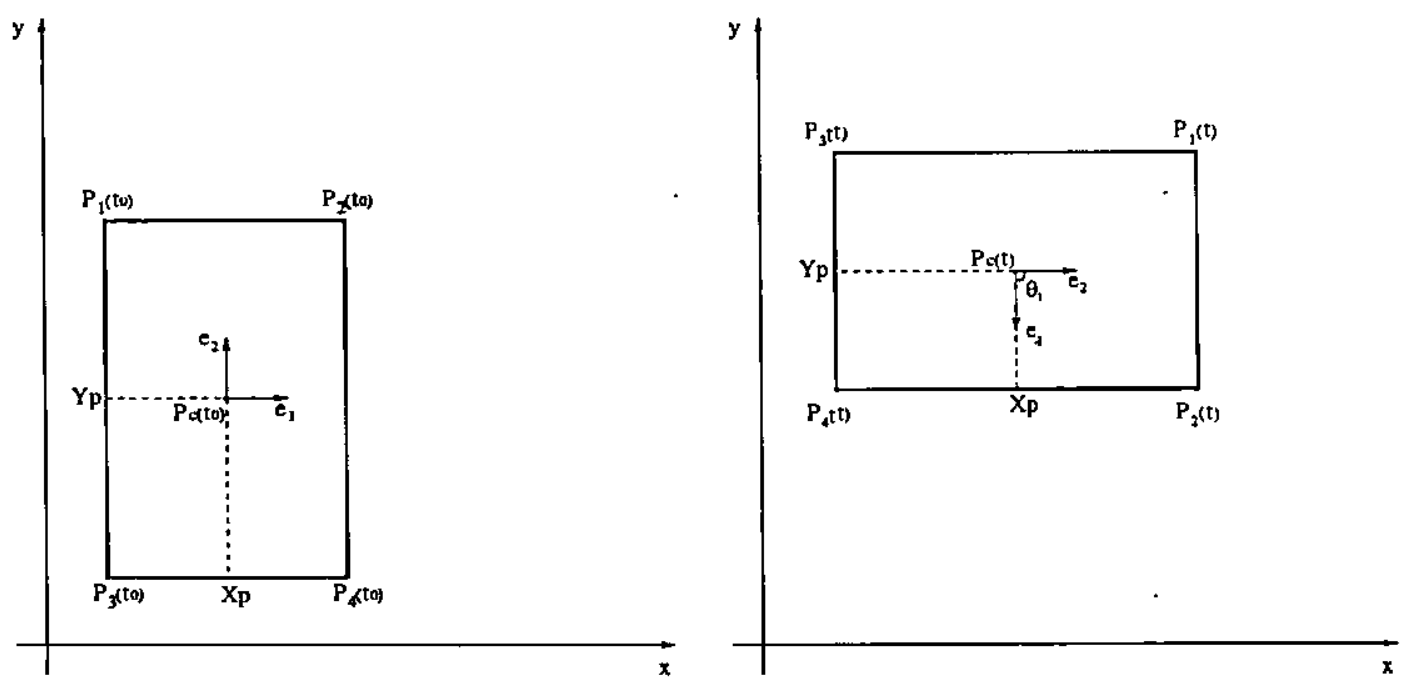

Figura 5.2: Objeto nos instantes $t_{0}$ e $t$

Neste exemplo observa-se o objeto após realizar um movimento de translação seguida de uma rotação em torno de seu centro de massa. A seguir estāo listados os dados que devem ser considerados:

- as coordenadas do centro de massa no instante $t$;

- o ângulo de rotação no instante $t$. 
Assim, calcula-se a posição dos vértices de acordo com

$$
\mathbf{P}_{i}(t)=\mathbf{P}_{c}(t)+\mathrm{A}\left(\theta_{t}\right)\left(\mathbf{P}_{i}\left(t_{0}\right)-\mathbf{P}_{c}\left(t_{0}\right)\right), \quad-\quad i=1,2,3,4
$$

onde $\mathrm{A}\left(\theta_{t}\right)$ é a matriz de rotação [15], definida por

$$
\mathbf{A}\left(\theta_{t}\right)=\left[\begin{array}{cc}
\cos \left(\theta_{t}\right) & -\sin \left(\theta_{t}\right) \\
\sin \left(\theta_{t}\right) & \cos \left(\theta_{t}\right)
\end{array}\right] .
$$

Obtém-se a velocidade do centro de massa derivando-se suas coordenadas em relação ao tempo:

$$
\mathrm{U}_{c}(t)=\mathrm{P}_{c}^{\prime}(t)
$$

ou

$$
\mathrm{U}_{c}(t)=\left(\begin{array}{c}
x_{c}^{\prime}(t) \\
y_{c}^{\prime}(t)
\end{array}\right)
$$

Evidentemente, a velocidade dos vértices do objeto é obtida, também, pela derivação de cada termo da equação 5.1 em relação ao tempo

$$
\mathrm{U}_{i}(t)=\mathrm{P}_{i}^{\prime}(t)
$$

ou seja

$$
\mathrm{U}_{i}=\mathrm{P}_{c}^{\prime}(t)+\mathbf{A}^{\prime}\left(\theta_{t}\right) \theta^{\prime}(t)\left(\mathbf{P}_{i}\left(t_{0}\right)-\mathbf{P}_{c}\left(t_{0}\right)\right)
$$

em que

$$
\mathbf{A}^{\prime}\left(\theta_{t}\right)=\left[\begin{array}{cc}
-\sin \left(\theta_{t}\right) & -\cos \left(\theta_{t}\right) \\
\cos \left(\theta_{t}\right) & -\sin \left(\theta_{t}\right)
\end{array}\right] .
$$

No decorrer da simulação, o objeto assume uma nova posição em cada instante de tempo. Com isso, não se pode recorrer ao instante de tempo $t_{0}$, já que esse dado não estará mais armazenado. A posição do objeto deve ser então calculada a partir do instante de tempo anterior. Assim, tem-se

$$
\mathrm{P}_{i}\left(t_{n}\right)=\mathrm{P}_{c}\left(t_{n}\right)+\mathbf{A}\left(\theta_{n}\right)\left(\mathrm{P}_{i}\left(t_{n-1}\right)-\mathrm{P}_{c}\left(t_{n-1}\right)\right)
$$

onde $\theta_{n}$ define o ângulo de rotação no tempo atual, dado por

$$
\theta_{n}=\theta\left(t_{n}\right)-\theta\left(t_{n-1}\right)
$$


c a velocidade é calculada segundo:

$$
\mathbf{U}_{i}\left(t_{n}\right)=\mathbf{P}_{c}^{\prime}\left(t_{n}\right)+\mathbf{A}^{\prime}\left(\theta_{n}\right) \theta^{\prime}\left(t_{n}\right)\left(\mathbf{P}_{i}\left(t_{n-1}\right)-\mathbf{P}_{c}\left(t_{n-1}\right)\right) .
$$

Sabendo-se que

$$
\mathbf{P}_{i}\left(t_{n-1}\right)-\mathbf{P}_{c}\left(t_{n-1}\right)=\mathbf{A}^{-1}\left(\theta_{n}\right)\left(\mathbf{P}_{i}\left(t_{n}\right)-\mathbf{P}_{c}\left(t_{n}\right),\right.
$$

a velocidade resulta em:

$$
\mathbf{U}_{i}\left(t_{n}\right)=\mathbf{P}_{c}^{\prime}\left(t_{n}\right)+\mathbf{A}^{\prime}\left(\theta_{n}\right) \theta^{\prime}\left(t_{n}\right) \mathbf{A}^{-1}\left(\theta_{n}\right)\left(\mathbf{P}_{i}\left(t_{n}\right)-\mathbf{P}_{c}\left(t_{n}\right)\right) .
$$

Tratando-se de um sistema interativo, o usuário poderá escolher o tipo de movimento que descreve a trajetória do objeto. Suponha-se que o objeto escolhido pelo usuário seja um contêiner do tipo Box inclinado, como mostra a figura 5.3.

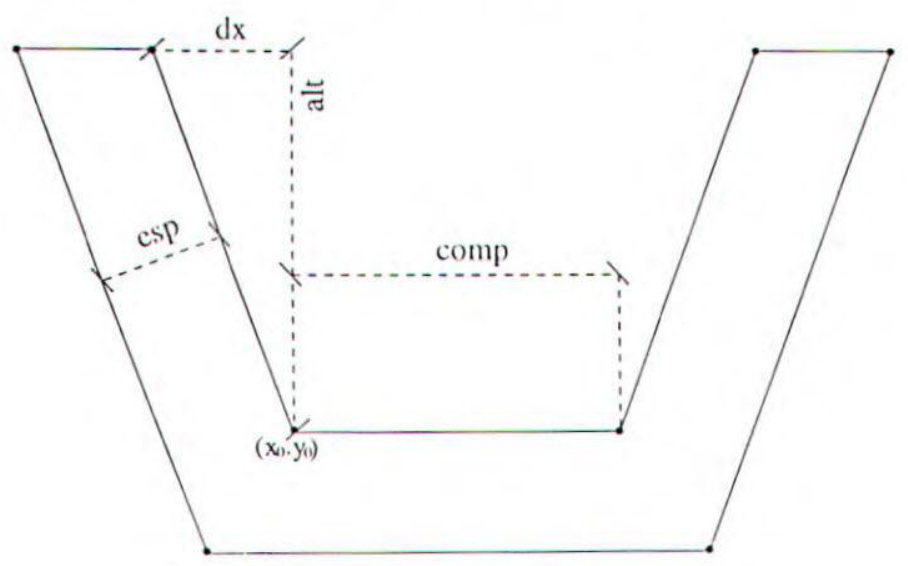

Figura 5.3: Contêiner do tipo Box inclinado

Os dados de entrada deste objeto são:

- nome do contêiner;

- tipo de contêiner;

- ponto de origem em x;

- ponto de origem em y;

- comprimento;

- altura;

- espessura;

- inclinação;

- plano; 
- orientação do contêiner;

- tipo de movimento.

Um contêiner do tipo Box reto apresenta ângulo de inclinação zero. Atualmente têm-se mais três tipos de contêineres implementados: Cube, Square-Torus e Box-Cilinder. O tipo de injetor padronizado é o Cubc. Os botões que definem os tipos de movimento foram criados na janela de entrada de dados de cada objeto, como mostra a figura 5.4.

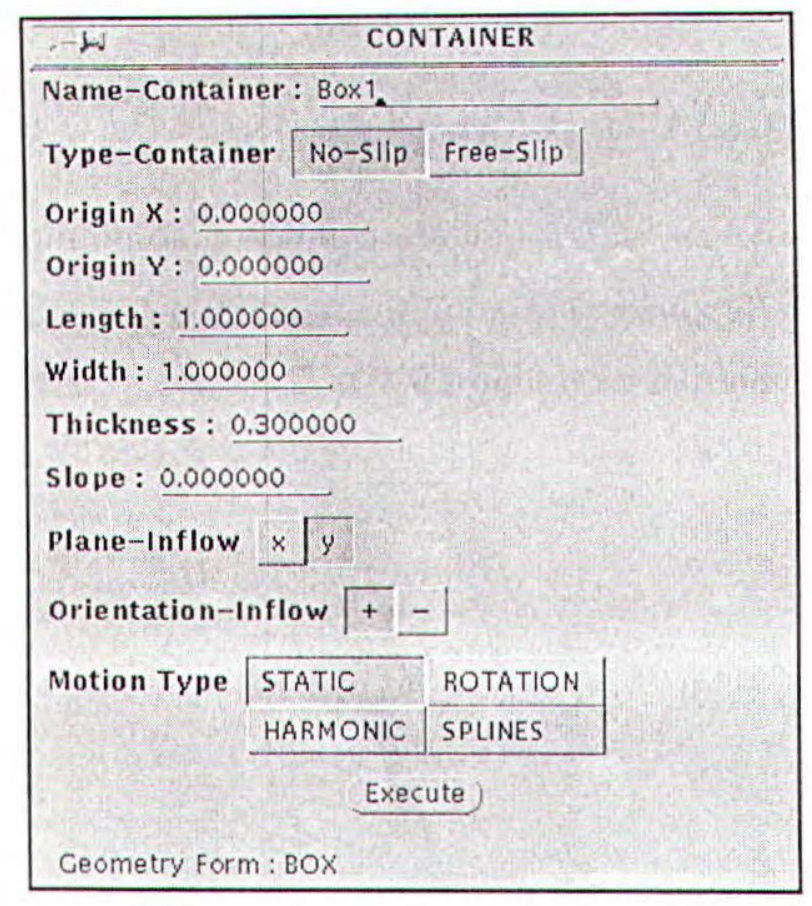

\begin{tabular}{l}
\hline INFLOW \\
Name-Inflow: Cube1. \\
Start-Inflow-Time : 0.000000 \\
Stop-Inflow-Time : 1.000000 \\
Velocity-Inflow: 1.000000 \\
Container-Thickness: 0.300000 \\
Type-Container $\sqrt{\text { No-Slip Free-Slip }}$ \\
Plane-Inflow $x \sqrt{y}$ \\
Orientation-Inflow + \\
Particles/Cell NX: \\
Origin X: 0.000000 \\
Origin Y: 0.000000 \\
Length: 1.000000 \\
Width: 1.000000 \\
Motion Type STATIC HARMONIC \\
Geometry Form: CUBE \\
\hline
\end{tabular}

Figura 5.4: Entrada de dados dos objetos

Três tipos de movimento foram implementados: rotação, harmônico simples e definido por interpolação por splines cúbica. De acordo com a figura 5.4, o movimento padrão (default) é o Estático. A seguir, apresenta-se cada tipo de movimento e sua interface gráfica. 


\subsubsection{Movimento de Rotação}

Considera-se o movimento de rotação do objeto em torno de seu centro de massa. Neste caso, a posição de $\mathrm{P}_{c}$, em qualquer instante $t$, permanece constante e sua velocidade torna-se nula:

$$
\mathbf{P}_{c}\left(t_{n}\right)=\mathbf{P}_{c}(t)=\left(\begin{array}{c}
x_{c} \\
y_{c}
\end{array}\right), \quad \mathbf{U}_{c}(t)=\left(\begin{array}{l}
0 \\
0
\end{array}\right) .
$$

O ângulo que define o movimento de rotação em torno do centro de massa é dado por

$$
\theta(t)=\frac{2 \pi}{T} t,
$$

onde $T$ (Cycle Time) é o tempo que o ângulo completa o ciclo de rotação, que pode ser escolhido pelo usuário, como mostra a figura 5.5. Outros dados que podem ser determinados pelo usuário é o tempo de início (Start Cycle Time) e de interrupção (Stop Cycle Time) do ciclo de rotação. Assim, o objeto só realiza o movimento de rotação durante o intervalo de tempo

$$
T_{\text {start }}<t<T_{\text {stop }}
$$

e sua posição, fora deste intervalo, permanece constante.

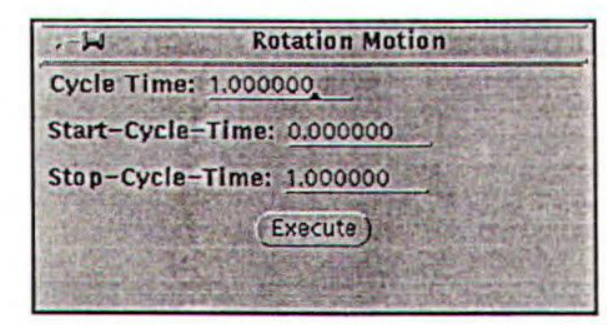

Figura 5.5: Movimento de Rotação

Esta janela aparece assim que, através do mouse, o usuário seleciona o tipo de movimento Rotation (figura 5.4). A velocidade do ângulo permanece constante durante a simulação:

$$
\theta^{\prime}(t)=\frac{2 \pi}{T}
$$

Conforme a equação 5.5 , a posição dos vértices é definida por

$$
\mathbf{P}_{i}\left(t_{n}\right)=\mathbf{P}_{c}\left(t_{n}\right)+\mathbf{A}\left(\theta_{n}\right)\left(\mathbf{P}_{i}\left(t_{n-1}\right)-\mathbf{P}_{c}\left(t_{n-1}\right)\right),
$$

em que

$$
\theta_{n}=\frac{2 \pi}{T} \Delta t, \quad \Delta t=t_{n}-t_{n-1} .
$$


Então,

$$
\mathbf{P}_{i}\left(t_{n}\right)=\left[\begin{array}{l}
x_{c}+\cos \left(\theta_{n}\right)\left(x_{i}\left(t_{n-1}\right)-x_{c}\right)-\sin \left(\theta_{n}\right)\left(y_{i}\left(t_{n-1}\right)-y_{c}\right) \\
y_{c}+\sin \left(\theta_{n}\right)\left(x_{i}\left(t_{n-1}\right)-x_{c}\right)+\cos \left(\theta_{n}\right)\left(y_{i}\left(t_{n-1}\right)-y_{c}\right)
\end{array}\right]
$$

e a velocidade em cada vértice é então dada pela equação

$$
\mathrm{U}_{i}\left(t_{n}\right)=\theta^{\prime}\left(t_{n}\right) \mathrm{A}^{\prime}\left(\theta_{n}\right) \mathbf{A}^{-1}\left(\theta_{n}\right)\left(\mathbf{P}_{i}\left(t_{n}\right)-\mathrm{P}_{c}\left(t_{n}\right)\right)
$$

Assim,

$$
\mathrm{U}_{i}\left(t_{n}\right)=\frac{2 \pi}{T}\left[\begin{array}{cc}
-\sin \left(\theta_{n}\right) & -\cos \left(\theta_{n}\right) \\
\cos \left(\theta_{n}\right) & -\sin \left(\theta_{n}\right)
\end{array}\right]\left[\begin{array}{cc}
\cos \left(\theta_{n}\right) & \sin \left(\theta_{n}\right) \\
-\sin \left(\theta_{n}\right) & \cos \left(\theta_{n}\right)
\end{array}\right]\left[\begin{array}{c}
x_{i}\left(t_{n}\right)-x_{c} \\
y_{i}\left(t_{n}\right)-y_{c}
\end{array}\right]
$$

ou seja

$$
\mathrm{U}_{i}\left(t_{n}\right)=\frac{2 \pi}{T}\left[\begin{array}{c}
y_{c}-y_{i}\left(t_{n}\right) \\
x_{i}\left(t_{n}\right)-x_{c}
\end{array}\right]
$$

\subsubsection{Movimento Harmônico Simples}

Neste movimento o centro de massa do objeto desloca-se sobre a reta que une os pontos $\mathrm{P}_{c_{i}}$ e $\mathrm{P}_{c_{f}}$, onde $\mathrm{P}_{c_{i}}$ é o centro de massa do objeto no instante inicial, e $\mathrm{P}_{c_{f}}$ um ponto aleatório determinado pelo usuário, cujas coordenadas são constantes:

$$
\mathrm{P}_{c_{f}}=\left(\begin{array}{c}
x_{c_{f}} \\
y_{c_{f}}
\end{array}\right)
$$

Combinando a função seno com uma equação da reta que passa pelos pontos $\mathrm{P}_{c_{i}}$ e $\mathrm{P}_{c_{f}}$, obtém-se:

$$
\mathrm{P}_{c}(t)=\frac{1}{2}\left(\mathrm{P}_{c_{f}}-\mathrm{P}_{c_{i}}\right) \sin \left(-\frac{\pi}{2}+\frac{\pi}{T} t\right)+\frac{1}{2}\left(\mathrm{P}_{c_{i}}+\mathrm{P}_{c_{f}}\right)
$$

E portanto, o centro de massa descreve um movimento harmônico simples, transladado em relação à origem, ou seja, o centro de massa oscila entre os pontos $\mathrm{P}_{c_{i}}$ e $\mathrm{P}_{c_{f}}$ durante o tempo da simulação. A constante $T$ (Cycle Time) é o período necessário para uma oscilação completa, o tempo em que o centro de massa leva para se deslocar do ponto $\mathrm{P}_{c_{i}}$ ao ponto $\mathrm{P}_{c_{f}}$, ou vice-versa. Esta constante pode ser escolhida pelo usuário, juntamente com os instantes de tempo inicial (Start Cycle Time) e final (Stop Cycle Time) do ciclo de oscilação, como mostra a figura 5.6. 


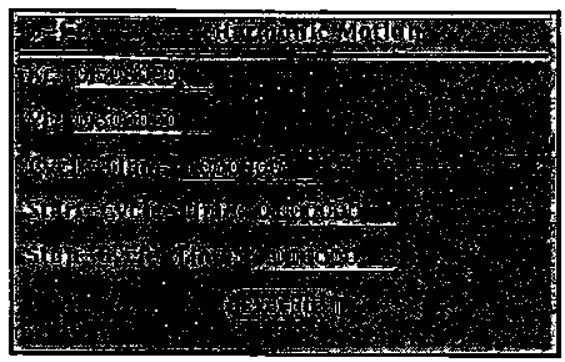

Figura 5.6: Movimento Harmônico Simples

As coordenadas do ponto $\mathbf{P}_{c_{f}}$ são iguais as coordenadas do centro de massa no instante inicial. Esse dado é tido como dado padrão inicial (default).

A equação 5.11 também pode ser escrita como

$$
\mathbf{P}_{c}\left(t_{n}\right)=\left[\begin{array}{c}
\frac{1}{2}\left(x_{c_{f}}-x_{c_{i}}\right) \sin \left(-\frac{\pi}{2}+\frac{\pi}{T} t_{n}\right)+\frac{1}{2}\left(x_{c_{i}}+x_{c_{f}}\right) \\
\frac{1}{2}\left(y_{c_{f}}-y_{c_{i}}\right) \sin \left(-\frac{\pi}{2}+\frac{\pi}{T} t_{n}\right)+\frac{1}{2}\left(y_{c_{i}}+y_{c_{f}}\right)
\end{array}\right] .
$$

A velocidade do centro de massa é definida por

$$
\mathrm{U}_{c}\left(t_{n}\right)=\frac{\pi}{2 T}\left(\mathrm{P}_{c_{f}}-\mathrm{P}_{c_{i}}\right) \cos \left(-\frac{\pi}{2}+\frac{\pi}{T} t_{n}\right)
$$

ou

$$
\mathrm{U}_{c}\left(t_{n}\right)=\left[\begin{array}{c}
\frac{\pi}{2 T}\left(x_{c_{f}}-x_{c_{i}}\right) \cos \left(-\frac{\pi}{2}+\frac{\pi}{T} t_{n}\right) \\
\frac{\pi}{2 T}\left(y_{c_{f}}-y_{c_{i}}\right) \cos \left(-\frac{\pi}{2}+\frac{\pi}{T} t_{n}\right)
\end{array}\right] .
$$

Durante este movimento, o centro de massa, juntamente com os vẹ́rtices, não realiza o movimento de rotação e portanto, o ângulo $\theta(t)$ permanece constante,

$$
\theta(t)=c t e \Rightarrow \theta_{n}=0 .
$$

Portanto, a matriz de rotação torna-se a matriz identidade I.

A posição dos vértices é calculada segundo

$$
\mathrm{P}_{i}\left(t_{n}\right)=\mathbf{P}_{c}\left(t_{n}\right)+\mathrm{I}\left(\mathrm{P}_{i}\left(t_{n-1}\right)-\mathrm{P}_{c}\left(t_{n-1}\right)\right)
$$

ou

$$
P_{i}\left(t_{n}\right)=\left[\begin{array}{c}
x_{c}\left(t_{n}\right)+x_{i}\left(t_{n-1}\right)-x_{c}\left(t_{n-1}\right) \\
y_{c}\left(t_{n}\right)+y_{i}\left(t_{n-1}\right)-y_{c}\left(t_{n-1}\right)
\end{array}\right] \text {. }
$$


De acordo com a equação 5.7 , a velocidade dos vértices é então

$$
\mathrm{U}_{i}\left(t_{n}\right)=\mathbf{P}_{c}^{\prime}\left(t_{n}\right)
$$

ou seja

$$
\mathrm{U}_{i}\left(t_{n}\right)=\frac{\pi}{2 T}\left(\mathrm{P}_{c_{f}}-\mathbf{P}_{c_{i}}\right) \cos \left(-\frac{\pi}{2}+\frac{\pi}{T} t_{n}\right)
$$

e obtém-se que a velocidade dos vértices é igual a velocidade do centro de massa, conforme a equação 5.13 .

\subsubsection{Movimento Definido por Interpolação por Splines Cúbica}

O usuário poderá escolher o tipo de movimento que o objeto vai realizar durante a simulação. Para isto, as coordenadas do centro de massa do objeto e seu ângulo serão calculados através de alguma técnica de aproximação.

Considera-se a interpolação por splines cúbica com condição de fronteira livre. As funções splines estão associadas a uma partição predeterminada do intervalo $[a, b]$, onde pretende-se trabalhar. Uma partição será definida pelos pontos $t_{0}, t_{1}, \cdots, t_{n}$ tais que

$$
a=t_{0}<t_{1}<t_{2}<\cdots<t<t_{n}=b
$$

Para usar as funções splines em interpolação tomamos os pontos de interpolação como nós da partição à qual associa-se as funções splines.

A splines cúbica caracteriza-se por uma "colagem" de polinômios de grau $3 \mathrm{em}$ cada subintervalo da partição, de modo que a função resultante tenha duas derivadas contínuas em $[a, b]$.

Para encontrar a splines cúbica para a função $f$, utiliza-se

$$
S_{i}(t)=f_{i} \varphi_{0 i}(t)+f_{i+1} \varphi_{1 i}(t)+f_{i}^{\prime} \psi_{0 i}(t)+f_{i+1}^{\prime} \psi_{1 i}(t)
$$

onde $\varphi_{0 i}(t), \varphi_{1 i}(t), \psi_{0 i}(t), \psi_{1 i}(t)$ são termos do polinômio de Hermite:

$$
\begin{aligned}
& \varphi_{0 i}(t)=\frac{1}{h_{i}^{2}}\left(t-t_{i+1}\right)^{2}\left(1+\frac{2}{h_{i}}\left(t-t_{i}\right)\right), \\
& \varphi_{1 i}(t)=\frac{1}{h_{i}^{2}}\left(t-t_{i}\right)^{2}\left(1-\frac{2}{h_{i}}\left(t-t_{i+1}\right)\right),
\end{aligned}
$$




$$
\begin{aligned}
& \psi_{0 i}(t)=\frac{1}{h_{i}^{2}}\left(t-t_{i+1}\right)^{2}\left(t-t_{i}\right), \\
& \psi_{1 i}(t)=\frac{1}{h_{i}^{2}}\left(t-t_{i}\right)^{2}\left(t-t_{i+1}\right) .
\end{aligned}
$$

Ao escolher o tipo de movimento definido pela fựção splines, o usuário deverá escolher qual a coordenada que deseja aproximar, como mostra a figura 5.7.

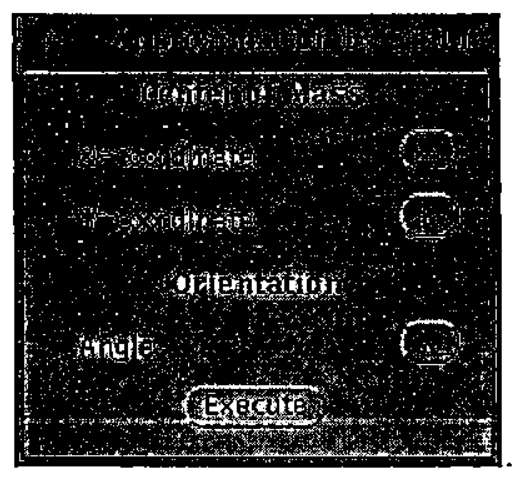

Figura 5.7: Aproximação por splines

Em seguida, o usuário deve escolher qual o modo de entrada dos dados, de acordo com a figura 5.8 .

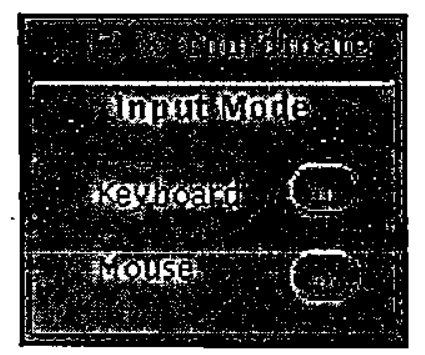

Figura 5.8: Entrada dos Dados

Tem-se duas opções, como mostra a figura 5.9: fornecer as coordenadas dos pontos de interpolação ou selecioná-los através do mouse. 

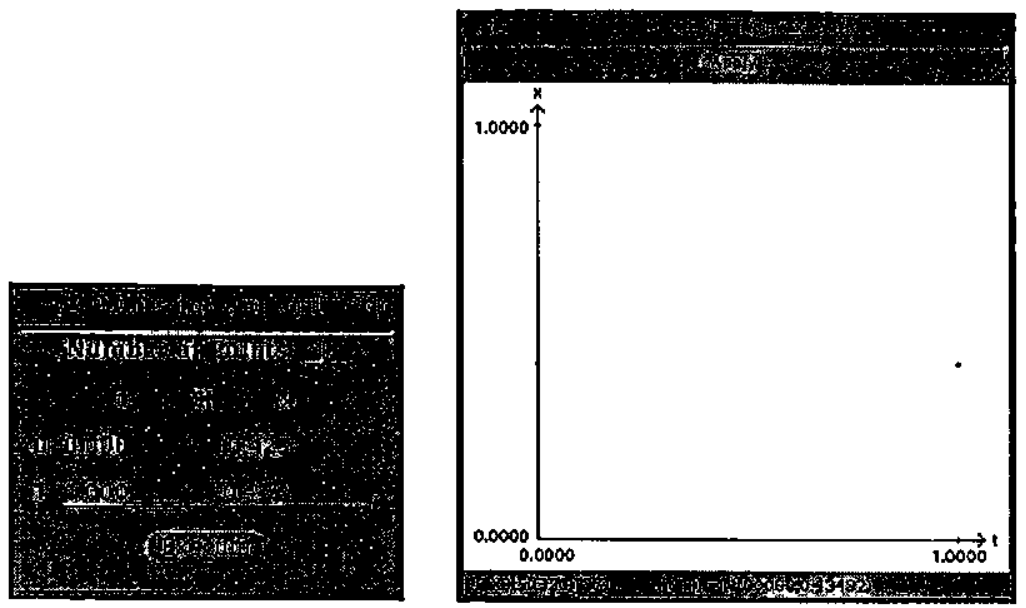

Figura 5.9: Pontos de Interpolação

Por default, o centro de massa de cada objeto permanece constante durante a simulação. A seguir, exemplificarse um tipo de movimento definido pelo usuário.

Considerarse um sistema de coordenadas cartesianas de $t$ em função de $x_{c}$. Suponhar se que o usuário èscolha os pontos de interpolação para a aproximação da coordenada $x_{c}$ pela funçāo splines củbica, conforme mostra a figura 5.10.
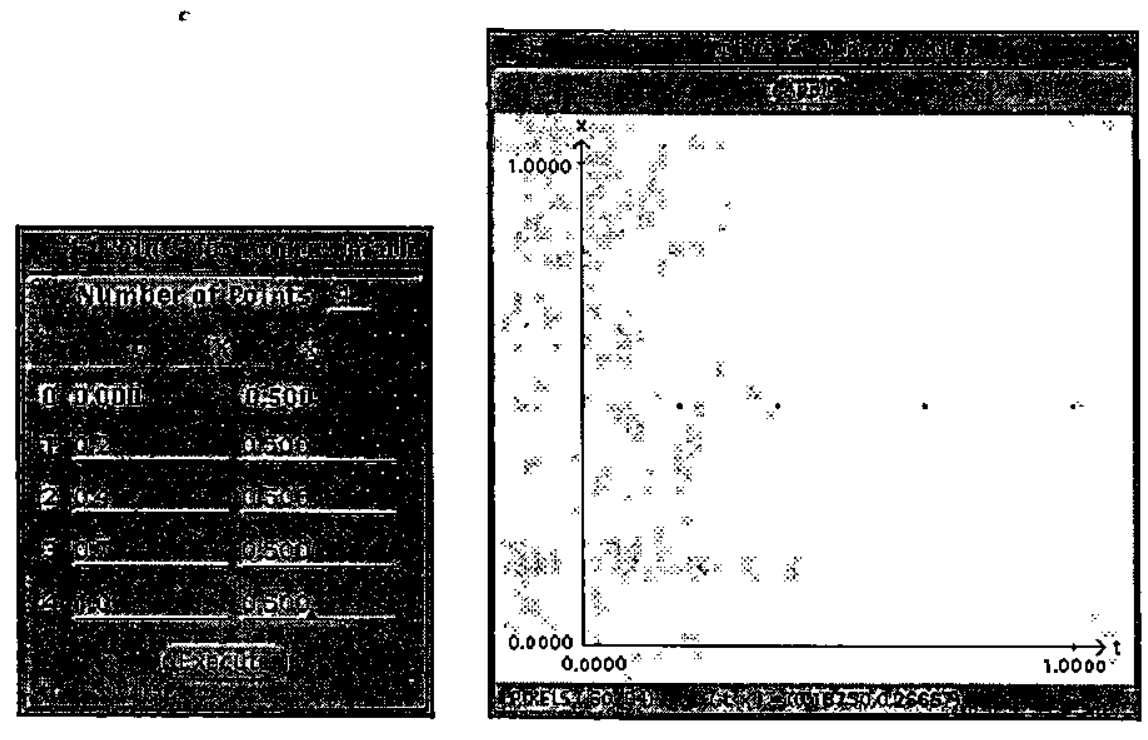

Figura 5.10: Interpolação por splines para $x_{c}$

De acordo com o polinômio 5.17 , obtém-se a função que aproxima esta coordenada no decorrer da simulaçāo.

Conforme os pontos de interpolação, a coordenada $x_{c}$ do centro de massa perma- 
nece constante para qualquer instante de tempo $t$. Assim, $x_{c}$ assume a seguinte forma:

$$
x_{c}(t)=x_{c}\left(t_{0}\right) \varphi_{0 i}(t)+x_{c}\left(t_{0}\right) \varphi_{1 i}(t)
$$

Em seguida considera-se o mesmo sistema de coordenadas cartesianas para a coordenada $y_{c}$, onde o usuário não determina pontos de interpolação. De acordo com os dados iniciais (default), a coordenada $y_{c}$ do centro de massa permanece constante durante a simulação e portanto, pode ser escrita de forma análoga a 5.18.

Logo, as coordenadas do centro de massa são:

$$
\mathrm{P}_{c}(t)=\left[\begin{array}{l}
x_{c}\left(t_{0}\right) \varphi_{0 i}(t)+x_{c}\left(t_{0}\right) \varphi_{1 i}(t) \\
y_{c}\left(t_{0}\right) \varphi_{0 i}(t)+y_{c}\left(t_{0}\right) \varphi_{1 i}(t)
\end{array}\right]
$$

A velocidade de $P_{c}$ é obtida pela derivação de suas coordenadas, ou seja

$$
\mathrm{U}_{c}\left(t_{n}\right)=\mathrm{P}_{c}^{\prime}\left(t_{n}\right)=\left(\begin{array}{l}
0 \\
0
\end{array}\right)
$$

Para o ângulo $\theta$ o processo é análogo. Considera-se um sistema de coordenadas cartesianas de $t$ em função de $\theta$ e suponha-se que o usuário escolha, por exemplo, os pontos de interpolação como mostra a figura 5.11 .
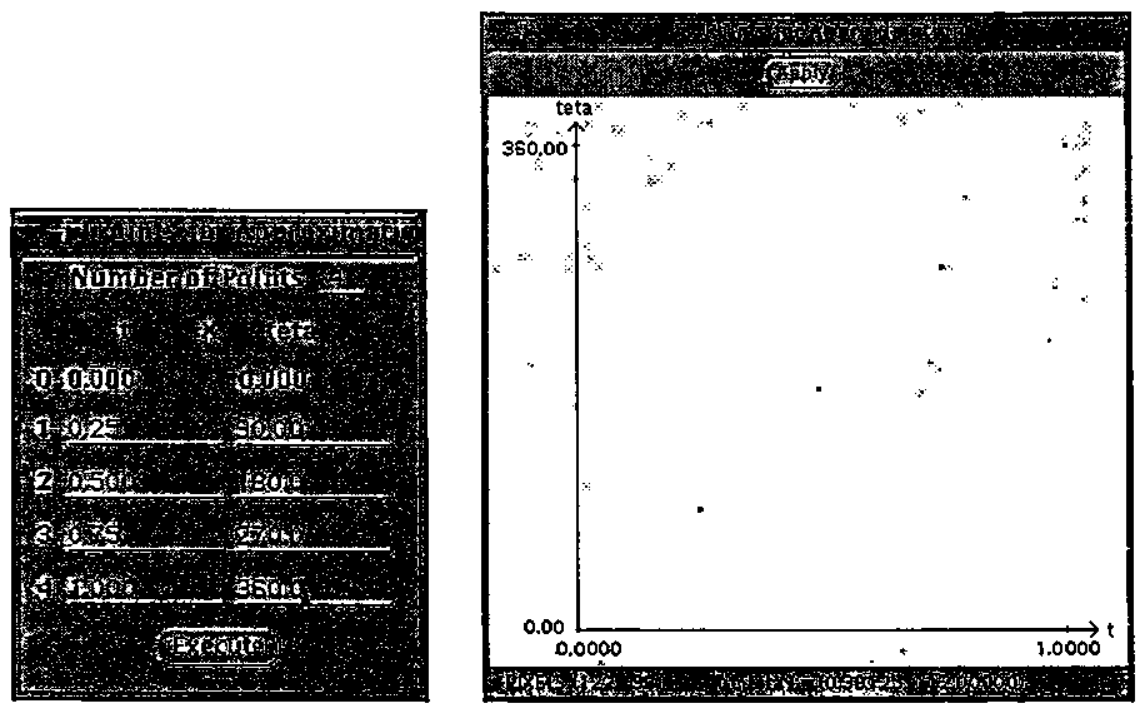

Figura 5.11: Interpolação por splines cúbica para $\theta$

Assim que estes pontos são escolhidos e o botāo Execute ou Apply for clicado pelo usuário, as coordenadas destes pontos são ordenadas de forma crescente, através de um 
método de ordenação denominado Método Quicksort [20].

De acordo com o polinômio 5.17 , obtém-se

$$
\begin{gathered}
\theta(t)=\frac{1}{h_{i}^{2}}\left[\left(t-t_{i+1}\right)^{2}\left(\theta_{i}+\left(t-t_{i}\right)\left(\frac{2 \theta_{i}}{h_{i}}+\theta_{i}^{\prime}\right)\right)+\right. \\
\left.\left(t-t_{i}\right)^{2}\left(\theta_{i+1}+\left(t-t_{i+1}\right)\left(\theta_{i+1}^{\prime}-\frac{2 \theta_{i+1}}{h_{i}}\right)\right)\right]
\end{gathered}
$$

que aproxima o ângulo $\theta$.

Observa-se que a função splines é uma função linear que aproxima o ângulo $\theta$. Assim, tem-se:

$$
\theta_{n}=S_{\theta}\left(t_{n}\right)-S_{\theta}\left(t_{n-1}\right)
$$

Especificamente neste caso, ocorre o movimento de rotação do objeto em torno de seu centro de massa, pois as coordenadas do centro de massa são constantes e o ângulo, uma função que depende do tempo. Uma observação importante é que para cada interpolação, o usuário poderá escolher a quantidade de pontos que achar necessário e estes pontos não precisam estar igualmente espaçados.

Durante este movimento, a posição dos vértices é definida por

$$
\mathrm{P}_{i}\left(t_{n}\right)=\mathrm{P}_{c}\left(t_{n}\right)+\mathrm{A}\left(\theta_{n}\right)\left(\mathrm{P}_{i}\left(t_{n-1}\right)-\mathrm{P}_{c}\left(t_{n-1}\right)\right)
$$

ou

$$
\mathbf{P}_{i}\left(t_{n}\right)=\left[\begin{array}{l}
x_{c}+\cos \left(\theta_{n}\right)\left(x_{i}\left(t_{n-1}\right)-x_{c}\right)-\sin \left(\theta_{n}\right)\left(y_{i}\left(t_{n-1}\right)-y_{c}\right) \\
y_{c}+\sin \left(\theta_{n}\right)\left(x_{i}\left(t_{n-1}\right)-x_{c}\right)+\cos \left(\theta_{n}\right)\left(y_{i}\left(t_{n-1}\right)-y_{c}\right)
\end{array}\right]
$$

e a velocidade em cada vértice é calculada segundo

$$
\mathrm{U}_{i}\left(t_{n}\right)=\mathrm{A}^{\prime}\left(\theta_{n}\right) \theta^{\prime}\left(t_{n}\right) \mathrm{A}^{-1}\left(\theta_{n}\right)\left(\mathrm{P}_{i}\left(t_{n}\right)-\mathrm{P}_{c}\left(t_{n}\right)\right)
$$

onde

$$
\theta^{\prime}\left(t_{n}\right)=S_{\theta}^{\prime}\left(t_{n}\right)
$$

Então, a velocidade dos vértices torna-se

$$
\mathrm{U}_{i}\left(t_{n}\right)=\theta^{\prime}\left(t_{n}\right)\left[\begin{array}{cc}
-\sin \left(\theta_{n}\right) & -\cos \left(\theta_{n}\right) \\
\cos \left(\theta_{n}\right) & -\sin \left(\theta_{n}\right)
\end{array}\right]\left[\begin{array}{cc}
\cos \left(\theta_{n}\right) & \sin \left(\theta_{n}\right) \\
-\sin \left(\theta_{n}\right) & \cos \left(\theta_{n}\right)
\end{array}\right]\left[\begin{array}{c}
x_{i}\left(t_{n}\right)-x_{c} \\
y_{i}\left(t_{n}\right)-y_{c}
\end{array}\right]
$$

ou seja

$$
\mathrm{U}_{i}\left(t_{n}\right)=\theta^{\prime}\left(t_{n}\right)\left[\begin{array}{c}
y_{c}-y_{i}\left(t_{n}\right) \\
x_{i}\left(t_{n}\right)-x_{c}
\end{array}\right]
$$


A seguir, apresenta-se outro exemplo de movimento definido por splines. Considerase os sistemas de eixos discutidos anteriormente. Suponha-se que o usuário escolha os pontos de interpolação, conforme mostra a figura 5.12, para a coordenada $x_{c}$ do centro de massa.
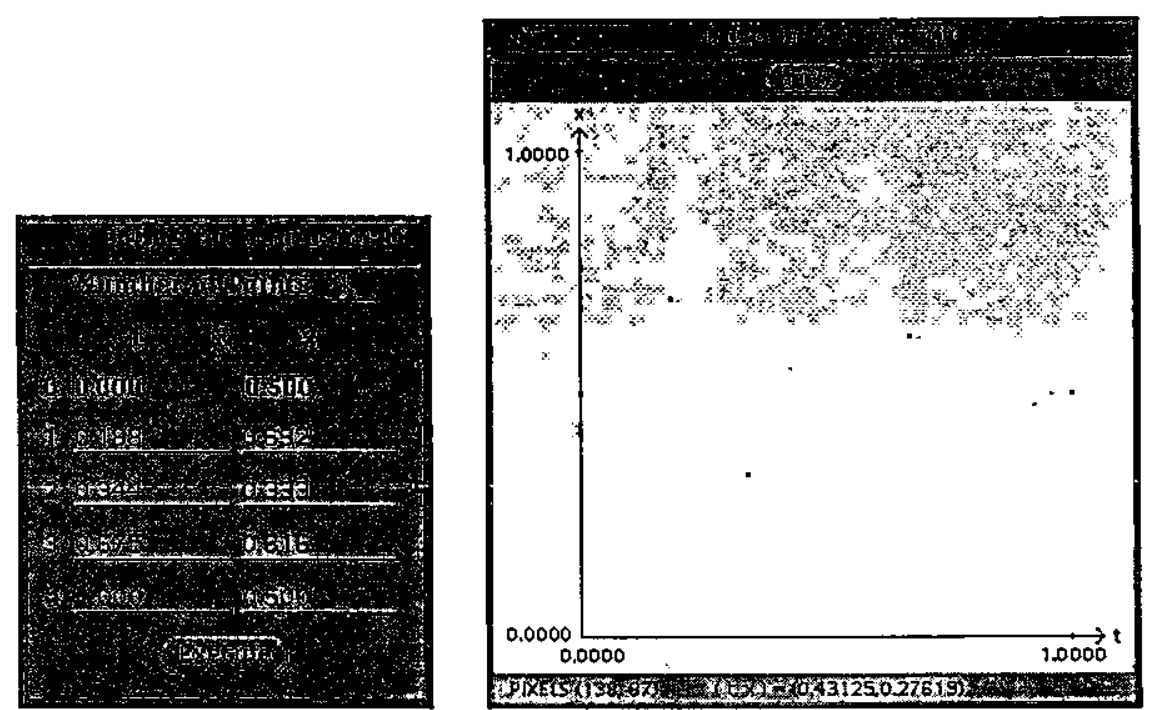

Figura 5.12: Interpolação por splines cúbica para $x_{c}$

Assim, de acordo com a equação 5.17 , obtém-se a aproximação pela função splines cúbica para a coordenada $x_{c}$, ou seja

$$
\begin{gathered}
x_{c}(t)=\frac{1}{h_{i}^{2}}\left[\left(t-t_{i+1}\right)^{2}\left(x_{c i}+\left(t-t_{i}\right)\left(\frac{2 x_{c i}}{h_{i}}+x_{c i}^{\prime}\right)\right)+\right. \\
\left.\left(t-t_{i}\right)^{2}\left(x_{c i+1}+\left(t-t_{i+1}\right)\left(x_{c i+1}^{\prime}-\frac{2 x_{c i+1}}{h_{i}}\right)\right)\right] .
\end{gathered}
$$

Agora em relação a $y_{c}$, suponha-se que o usuário escolha os pontos de interpolação conforme a figura 5.13 .

Assim, tem-se:

$$
\begin{gathered}
y_{c}(t)=\frac{1}{h_{i}^{2}}\left[\left(t-t_{i+1}\right)^{2}\left(y_{c_{i}}+\left(t-t_{i}\right)\left(\frac{2 y_{c_{i}}}{h_{i}}+y_{c i}^{\prime}\right)\right)+\right. \\
\left.\left(t-t_{i}\right)^{2}\left(y_{c_{i+1}}+\left(t-t_{i+1}\right)\left(y_{c i+1}^{\prime}-\frac{2 y_{c_{i+1}}}{h_{i}}\right)\right)\right] .
\end{gathered}
$$

Portanto, as coordenadas do centro de massa podem ser escritas de acordo com as equações 5.26 e 5.27 . 

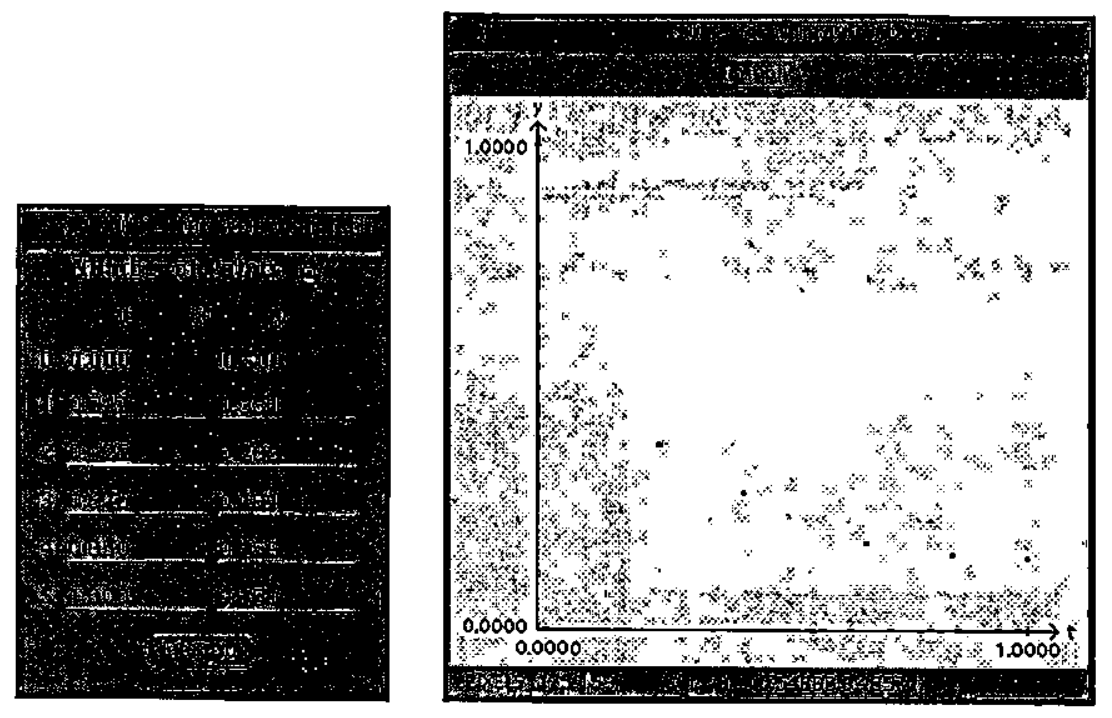

Figura 5.13: Interpolação por splines cúbica para $y_{c}$

A velocidade do centro de massa é obtida pela derivação de cada termo de suas coordenadas em relação ao tempo. Para a coordenada $x_{c}$ obtém-se:

$$
\begin{gathered}
x_{c}^{\prime}(t)=\frac{1}{h_{i}^{2}}\left[\left(t-t_{i+1}\right)^{2}\left(\frac{2 x_{c i}}{h_{i}}+x_{c i}^{\prime}\right)+\left(t-t_{i}\right)^{2}\left(x_{c i+1}^{\prime}-\frac{2 x_{c i+1}}{h_{i}}\right)+\right. \\
2\left(t-t_{i+1}\right)\left(x_{c i}+\frac{2 x_{c i}}{h_{i}}\left(t-t_{i}\right)\left(x_{c i}-x_{c i+1}\right)\right)+ \\
\left.2\left(t-t_{i}\right)\left(x_{c i+1}+\left(t-t_{i+1}\right)\left(x_{c i}^{\prime}+x_{c i+1}^{\prime}\right)\right)\right] .
\end{gathered}
$$

E analogamente, para a coordenada $y_{c}$ do centro de massa:

$$
\begin{gathered}
y_{c}^{\prime}(t)=\frac{1}{h_{i}^{2}}\left[\left(t-t_{i+1}\right)^{2}\left(\frac{2 y_{c i}}{h_{i}}+y_{c i}^{\prime}\right)+\left(t-t_{i}\right)^{2}\left(y_{c i+1}^{\prime}-\frac{2 y_{c i+1}}{h_{i}}\right)+\right. \\
2\left(t-t_{i+1}\right)\left(y_{c_{i}}+\frac{2 y_{c i}}{h_{i}}\left(t-t_{i}\right)\left(y_{c_{i}}-y_{c_{i+1}}\right)\right)+ \\
\left.2\left(t-t_{i}\right)\left(y_{c_{i+1}}+\left(t-t_{i+1}\right)\left(y_{c i}^{\prime}+y_{c i+1}^{\prime}\right)\right)\right] .
\end{gathered}
$$

Assim, durante simulação, a velocidade do centro de massa é calculada conforme as equações 5.28 e 5.29 .

Para a aproximação do ângulo $\theta$, o usuário poderá escolher apenas um ponto de interpolação, como mostra a figura 5.14. 


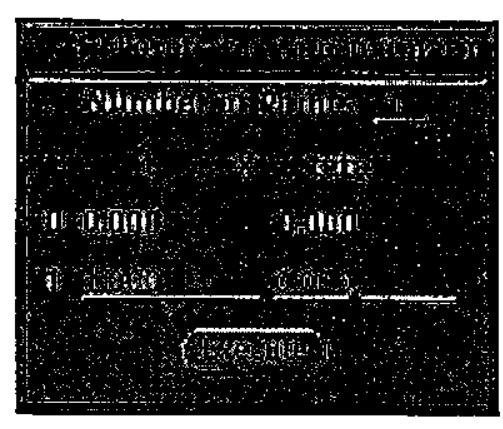

Figura 5.14: Interpolação por splines củbica para $\theta$

Obtém-se a aproximação para o ângulo, através da equação 5.21.

De acordo com os pontos de interpolação, pode-se notar que o ângulo $\theta$ permanece constante para qualquer instante de tempo $t$. Logo, $\theta$ assume a seguinte forma

$$
\theta(t)=\theta\left(t_{0}\right) \varphi_{0 i}(t)+\theta\left(t_{0}\right) \varphi_{1 i}(t)
$$

e

$$
\theta_{n}=\theta\left(t_{n}\right)-\theta\left(t_{n-1}\right)=0 .
$$

Este tipo de movimento definido pelo usuário é um caso particular de movimento de translação, em que a orientação do objeto não sofre alterações durante a simulaçāo. Deste modo, a posição atualizada dos vértices é definida de acordo com a equação 5.5 , ou seja

$$
\mathbf{P}_{i}\left(t_{n}\right)=\left[\begin{array}{l}
x_{c}\left(t_{n}\right)+x_{i}\left(t_{n-1}\right)-x_{c}\left(t_{n-1}\right) \\
y_{c}\left(t_{n}\right)+y_{i}\left(t_{n-1}\right)-y_{c}\left(t_{n-1}\right)
\end{array}\right]
$$

e a velocidade torna-se igual a velocidade do centro de massa.

De um modo geral, o movimento definido pelo usuário através da aproximação por splines cúbica, obedece as seguintes etapas:

i) Dados os pontos para a aproximação de cada coordenada, estes pontos são ordenados pelo método Quicksort;

ii) Durante a simulação, calcula-se o polinômio 5.17 para cada coordenada a ser aproximada e, em seguida, a posição atualizada do objeto de acordo, com a equação:

$$
\mathrm{P}_{i}\left(t_{n}\right)=\mathrm{P}_{c}\left(t_{n}\right)+\mathrm{A}\left(\theta_{n}\right)\left(\mathrm{P}_{i}\left(t_{n-1}\right)-\mathrm{P}_{c}\left(t_{n-1}\right)\right)
$$


A velocidade dos vértices é obtida segundo

$$
\mathrm{U}_{i}\left(t_{n}\right)=\mathrm{P}_{c}^{\prime}\left(t_{n}\right)+\mathbf{A}^{\prime}\left(\theta_{n}\right) \theta^{\prime}\left(t_{n}\right) \mathbf{A}^{-1}\left(\theta_{n}\right)\left(\mathrm{P}_{i}\left(t_{n}\right)-\mathrm{P}_{c}\left(t_{n}\right)\right)
$$

onde

$$
\mathrm{P}_{c}^{\prime}\left(t_{n}\right)=S_{c}^{\prime}\left(t_{n}\right)
$$

e

$$
\theta^{\prime}\left(t_{n}\right)=S_{\theta}^{\prime}\left(t_{n}\right)
$$

\subsection{O Modelador de Movimentos Tridimensional}

No modelador de movimentos tridimensional a trajetória dos objetos também será definida a partir do centro de massa:

$$
\mathrm{P}_{c}=\left(\begin{array}{l}
x_{c}(t) \\
y_{c}(t) \\
z_{c}(t)
\end{array}\right)
$$

As funções $x_{c}, y_{c}$ e $z_{c}$ são funções de classe $C^{2}$.

A seguir, a figura 5.15 mostra um objeto pré-definido que poderá ser escolhido pelo usuário, onde $P_{c}$ e $P_{i}, i=1, . ., 8$, representam o centro de massa e os vértices do objeto no instante inicial $-t_{0}$.

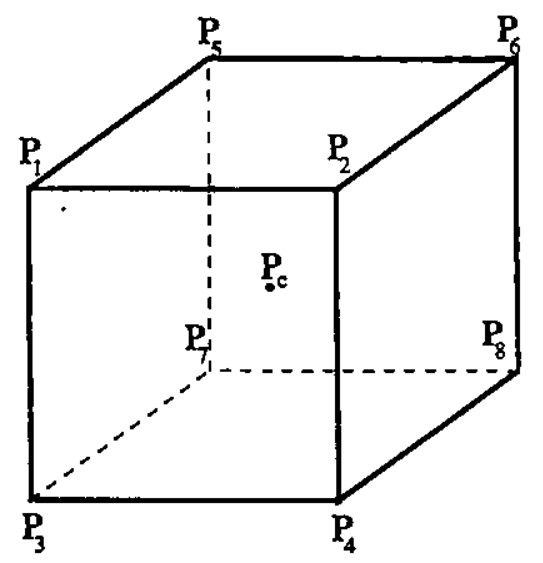

Figura 5.15: Objeto no instante $t_{0}$

Quanto a orientação, necessita-se de pelo menos dois ângulos para definir o objeto 
no espaço, diferente do caso bidimensional, onde apenas um ângulo se faz nécessário. Os seguintes ângulos serão utilizados:

$$
\theta_{1}=f_{1}(t), \quad \theta_{2}=f_{2}(t) \quad \text { e } \quad \varphi=g(t)
$$

em que os ângulos $\theta_{1}, \theta_{2}, \varphi$ são chamados ângulos de Euler em $R^{3}$ [4]. Estes ângulos definem o subespaço tridimensional o qual é ortogonal a direção de observação.

As matrizes de rotação para os ângulos de Euler são dadas por:

$$
\begin{aligned}
& \mathrm{R}_{12}^{3}\left(\theta_{1}\right)=\left[\begin{array}{ccc}
\cos \left(\theta_{1}\right) & -\sin \left(\theta_{1}\right) & 0 \\
\sin \left(\theta_{1}\right) & \cos \left(\theta_{1}\right) & 0 \\
0 & 0 & 1
\end{array}\right], \\
& \mathrm{R}_{23}^{3}\left(\theta_{2}\right)=\left[\begin{array}{ccc}
1 & 0 & 0 \\
0 & \cos \left(\theta_{2}\right) & -\sin \left(\theta_{2}\right) \\
0 & \sin \left(\theta_{2}\right) & \cos \left(\theta_{2}\right)
\end{array}\right], \\
& \mathrm{R}_{12}^{2}(\varphi)=\left[\begin{array}{ccc}
\cos (\varphi) & -\sin (\varphi) & 0 \\
\sin (\varphi) & \cos (\varphi) & 0 \\
0 & 0 & 1
\end{array}\right] .
\end{aligned}
$$

\subsubsection{Definição do Movimento de Translação e Rotação a partir do Centro de Massa}

Analogamente ao caso bidimensional, os objetos assumem uma nova posiçāo de acordo com o tempo durante a simulação. Para determinar a posição atual do objeto, precisa-se primeiro determinar as coordenadas do centro de massa e seus ângulos, de acordo com o tipo de movimento a ser descrito pelo objeto. Com isso, pode-se calcular a posição atualizada de seus vértices

$$
\mathbf{P}_{i}(t)=\left(\begin{array}{c}
x_{i}(t) \\
y_{i}(t) \\
z_{i}(t)
\end{array}\right)
$$


Considera-se um objeto que se desloca conforme mostra a figura 5.16.
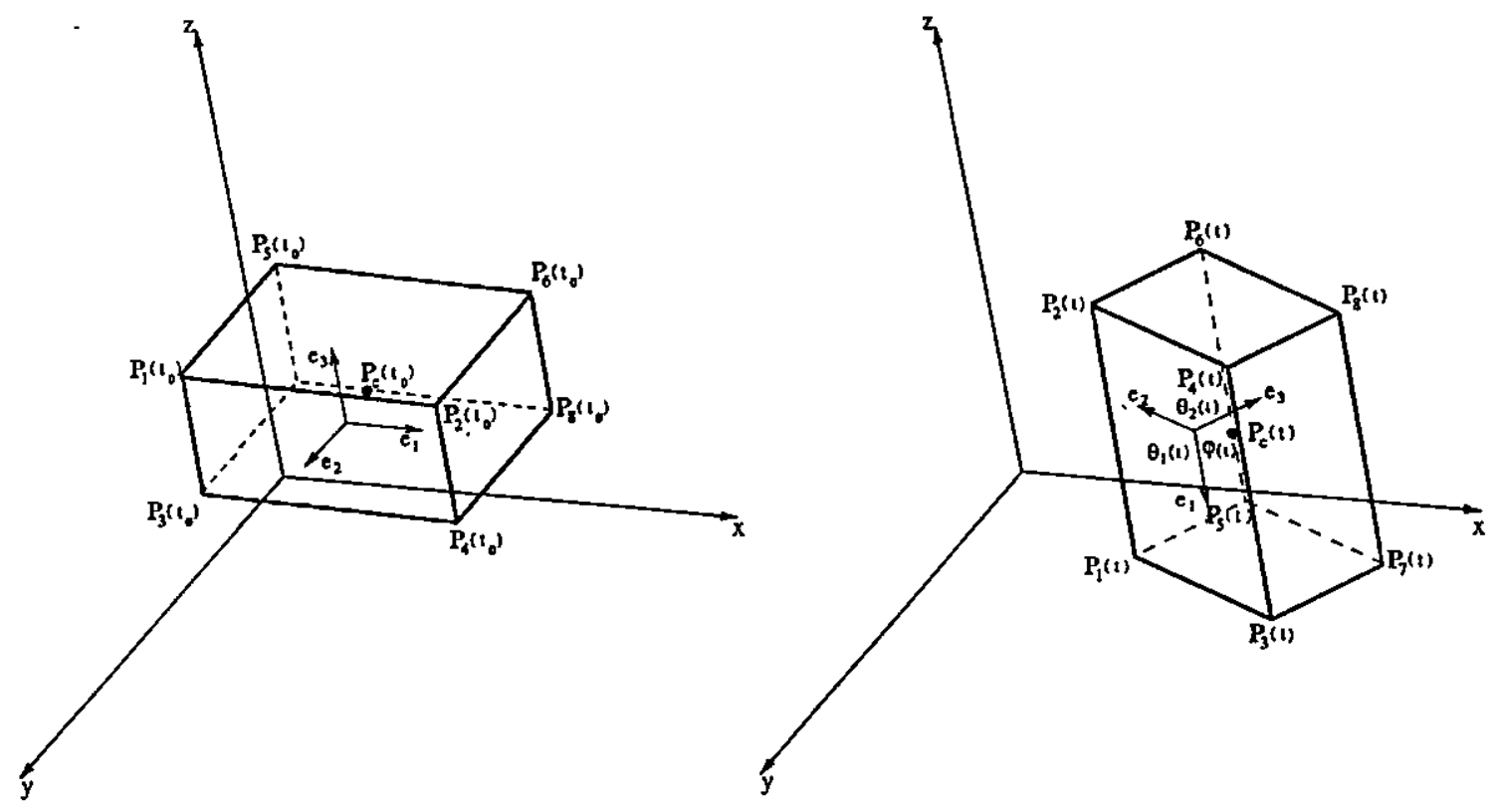

Figura 5.16: Objeto nos instantes $t_{0}$ e $t$

Este objeto realizou um movimento de translação seguido de uma rotação em torno de seu centro de massa. Os dados que devem ser determinados são:

- as coordenadàs do centro de massa no instante $t$;

- os ângulos de rotação no instante $t$.

Calcula-se a posição dos vértices do objeto de acordo com

$$
\mathbf{P}_{i}(t)=\mathbf{P}_{c}(t)+\mathbf{A}\left(\theta_{1}, \theta_{2}, \varphi\right)\left(\mathbf{P}_{i}\left(t_{0}\right)-\mathbf{P}_{c}\left(t_{0}\right)\right), \quad i=1,2,3,4,5,6,7,8
$$

onde a matriz $\mathbf{A}\left(\theta_{1}, \theta_{2}, \varphi\right)$ representa o produto de matrizes de rotação

$$
\mathrm{R}_{12}^{3}\left(\theta_{1}\right) \mathrm{R}_{23}^{3}\left(\theta_{2}\right) \mathrm{R}_{12}^{2}(\varphi)
$$

Obtém-se a velocidade do centro de massa derivando-se suas coordenadas em relação ao tempo e a velocidade dos vértices do objeto é obtida, também, pela derivação de cada termo da equação 5.1 em relação ao tempo

$$
\mathrm{U}_{i}(t)=\mathrm{P}_{i}^{\prime}(t)
$$

ou seja

$$
\mathrm{U}_{i}=\mathrm{P}_{c}^{\prime}(t)+\mathbf{A}^{\prime}\left(\theta_{1}, \theta_{2}, \varphi\right) \theta_{1}^{\prime} \theta_{2}^{\prime} \varphi^{\prime}\left(\mathrm{P}_{i}\left(t_{0}\right)-\mathrm{P}_{c}\left(t_{0}\right)\right)
$$


No decorrer da simulação, o objeto assume uma nova posição em cada instante de tempo. Com isso, não se pode recorrer ao instante de tempo $t_{0}$, já que esse dado não estará mais armazenado por causa do custo computacional. Os ângulos de rotação e a posição do objeto, em qualquer tempo, devem ser calculados a partir do instante de tempo anterior.

Para tanto, tem-se

$$
\mathbf{P}_{i}\left(t_{n}\right)=\mathbf{P}_{c}\left(t_{n}\right)+\mathbf{A}\left(\theta_{1 n}, \theta_{2 n}, \varphi_{n}\right)\left(\mathbf{P}_{i}\left(t_{n-1}^{-}\right)-\mathbf{P}_{c}\left(t_{n-1}\right)\right)
$$

e os ângulos de rotação no tempo atual:

$$
\begin{gathered}
\theta_{1 n}=\theta_{1}\left(t_{n}\right)-\theta_{1}\left(t_{n-1}\right), \\
\theta_{2 n}=\theta_{2}\left(t_{n}\right)-\theta_{2}\left(t_{n-1}\right), \\
\varphi_{n}=\varphi\left(t_{n}\right)-\varphi\left(t_{n-1}\right) .
\end{gathered}
$$

A velocidade pode ser expressa por:

$$
\mathrm{U}_{i}\left(t_{n}\right)=\mathbf{P}_{c}^{\prime}\left(t_{n}\right)+\mathbf{A}^{\prime}\left(\theta_{1 n}, \theta_{2 n}, \varphi_{n}\right) \theta_{1}^{\prime}\left(t_{n}\right) \theta_{2}^{\prime}\left(t_{n}\right) \varphi^{\prime}\left(t_{n}\right)\left(\mathbf{P}_{i}\left(t_{n-1}\right)-\mathbf{P}_{c}\left(t_{n-1}\right)\right)
$$

Sābendo-se que $\mathbf{A}\left(\theta_{1 \bar{n}}, \theta_{2 n}, \varphi_{n}\right)$.è um produto de matrizes ortogònais [15] e -

$$
\mathbf{P}_{i}\left(t_{n-1}\right)-\mathbf{P}_{c}\left(t_{n-1}\right)=\mathbf{A}^{-1}\left(\theta_{1 n}, \theta_{2 n}, \varphi_{n}\right)\left(\mathbf{P}_{i}\left(t_{n}\right)-\mathbf{P}_{c}\left(t_{n}\right)\right)
$$

a velocidade dos vértices é calculada segundo:

$$
\mathrm{U}_{i}\left(t_{n}\right)=\mathbf{P}_{c}^{\prime}\left(t_{n}\right)+\mathbf{A}^{\prime}\left(\theta_{1 n}, \theta_{2 n}, \varphi_{n}\right) \theta_{1}^{\prime}\left(t_{n}\right) \theta_{2}^{\prime}\left(t_{n}\right) \varphi^{\prime}\left(t_{n}\right) \mathbf{A}^{-1}\left(\theta_{1 n}, \theta_{2 n}, \varphi_{n}\right)\left(\mathbf{P}_{i}\left(t_{n}\right)-\mathbf{P}_{c}\left(t_{n}\right)\right)
$$

Em se tratando de um sistema interativo, o usuário poderá escolher o tipo de movimento que descreve a trajetória do objeto. Suponha-se que o objeto escolhido pelo usuário seja um contêiner do tipo caixa retangular sem tampa (Open Box), como mostra a figura 5.17. 


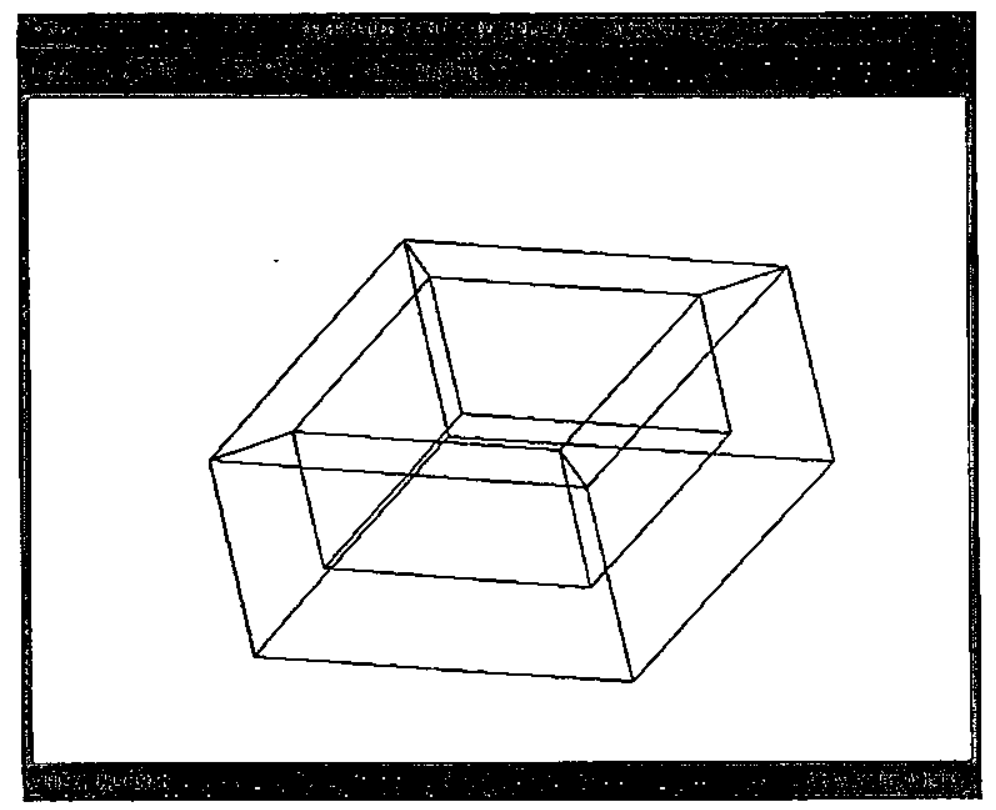

Figura 5.17: Contêiner do tipo Open Box

Os dados de entrada deste objeto são: nome do contêiner, tipo de contêiner, origem em $\mathrm{x}$, origem em $\mathrm{y}$, origem em $\mathrm{z}$, comprimento, largura, altura, espessura, inclinação e tipo de movimento, como mostra a figura 5.18.

Além de contêineres do tipo. Open Box, têm-se mais oito tipos implementados

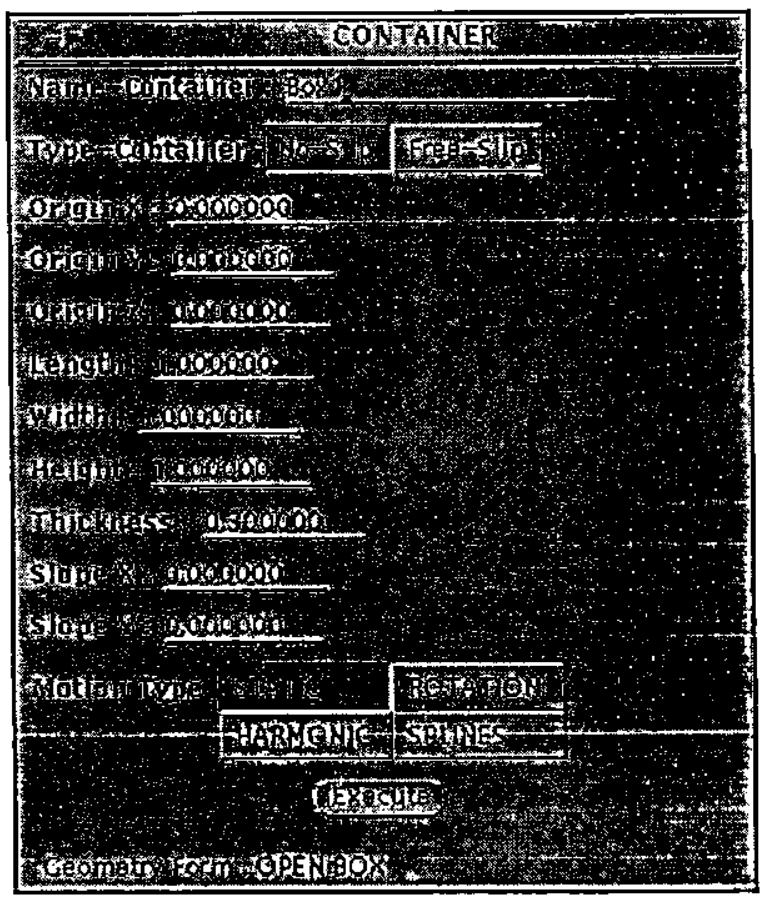

Figura 5.18: Entrada de dados de um contêiner do tipo Open Box 
atualmente, como mostra a figura 5.19.

Em relação aos injetores, tem-se somiente dois tipos: o Cube e o Cylinder. A

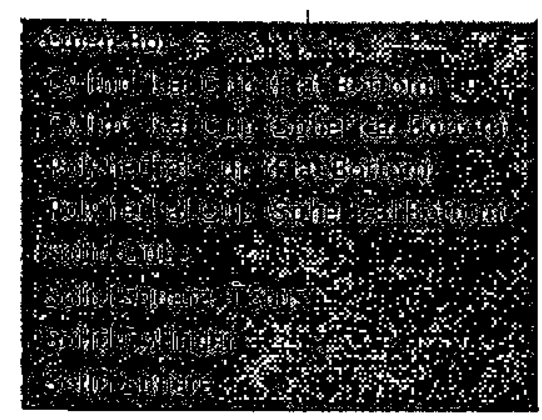

Figura 5.19: Tipos de contêineres

janela de entrada de dados de um injetor do tipo Cylinder é mostrada na figura 5.20.

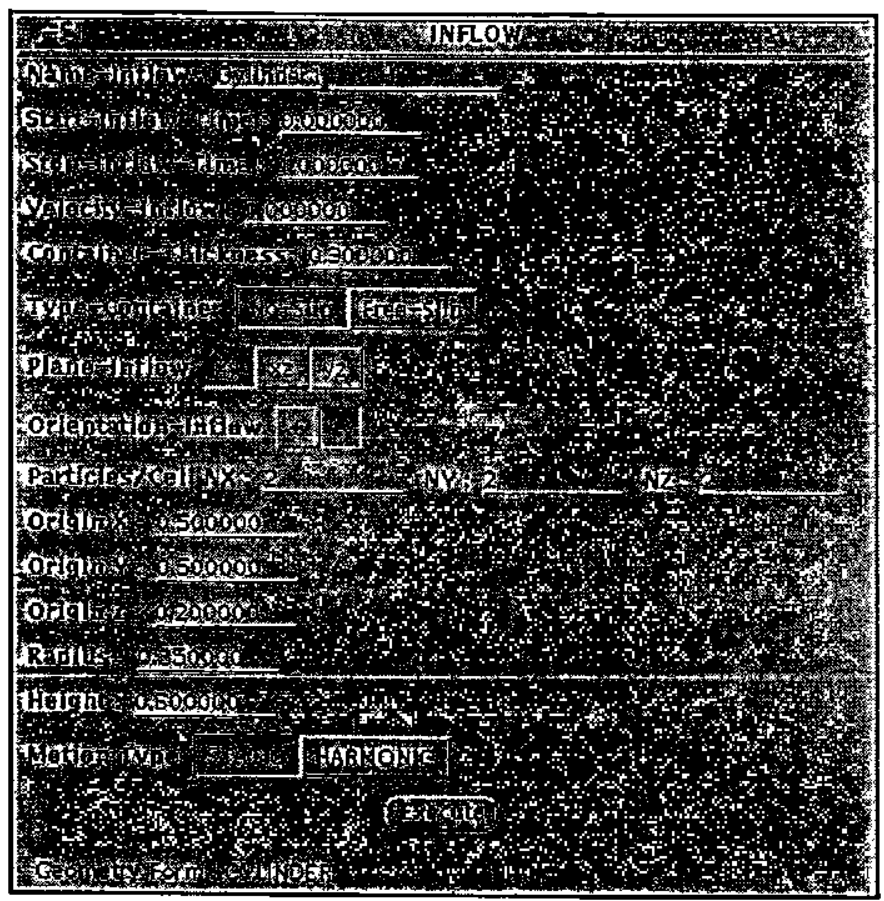

Figura 5.20: Entrada de dados de um injetor do tipo Cylinder

A seguir, apresenta-se cada tipo de movimento e sua interface gráfica. 


\subsubsection{Movimento de Rotação}

Utilizando-se os ângulos de Euler, pode-se definir o movimento de rotação em qualquer plano. Desse modo, tem-se três planos de rotação:

- Plano $x y$ : o movimento do objeto vai depender do ângulo $\theta_{1 n}$, fazendo-se os demais ângulos iguais a zero. Decorre deste fato que as matrizes $\mathbf{R}_{23}^{3}\left(\theta_{2 n}\right)$ e $\mathbf{R}_{12}^{2}\left(\varphi_{n}\right)$ tornam-se matrizes identidade I. Assim, o produto de matrizes de rotação torna-se

$$
A\left(\theta_{1 n}\right)=\left[\begin{array}{ccc}
\cos \left(\theta_{1 n}\right) & -\sin \left(\theta_{1 n}\right) & 0 \\
\sin \left(\theta_{1 n}\right) & \cos \left(\theta_{1 n}\right) & 0 \\
0 & 0 & 1
\end{array}\right]
$$

onde

$$
\theta_{1 n}=\frac{2 \pi}{T}\left(t_{n}-t_{n-1}\right)
$$

e $T$ o tempo do ciclo de rotação.

De acordo com a matriz de rotação para o plano $x y$, tem-se

$$
\mathrm{P}_{i}\left(t_{n}\right)=\left[\begin{array}{c}
x_{c}+\cos \left(\theta_{1 n}\right)\left(x_{i}\left(t_{n-1}\right)-x_{c}\right)-\sin \left(\theta_{1 n}\right)\left(y_{i}\left(t_{n-1}\right)-y_{c}\right) \\
y_{c}+\sin \left(\theta_{1 n}\right)\left(x_{i}\left(t_{n-1}\right)-x_{c}\right)+\cos \left(\theta_{1 n}\right)\left(y_{i}\left(t_{n-1}\right)-y_{c}\right) \\
z_{i}\left(t_{n-1}\right)
\end{array}\right]
$$

e'a velocidade em cada vértice é então dada pela equação

$$
\mathrm{U}_{i}\left(t_{n}\right)=\theta_{1}^{\prime}\left(t_{n}\right) \mathrm{A}^{\prime}\left(\theta_{1 n}\right) \mathrm{A}^{-1}\left(\theta_{1 n}\right)\left(\mathbf{P}_{i}\left(t_{n}\right)-\mathbf{P}_{c}\left(t_{n}\right)\right)
$$

Assim,

$$
\mathrm{U}_{i}\left(t_{n}\right)=\frac{2 \pi}{T}\left[\begin{array}{ccc}
-\sin \left(\theta_{1 n}\right) & -\cos \left(\theta_{1 n}\right) & 0 \\
\cos \left(\theta_{1 n}\right) & -\sin \left(\theta_{1 n}\right) & 0 \\
0 & 0 & 0
\end{array}\right]\left[\begin{array}{ccc}
\cos \left(\theta_{1 n}\right) & \sin \left(\theta_{1 n}\right) & 0 \\
-\sin \left(\theta_{1 n}\right) & \cos \left(\theta_{1 n}\right) & 0 \\
0 & 0 & 1
\end{array}\right]\left[\begin{array}{c}
x_{i}\left(t_{n}\right)-x_{c} \\
y_{i}\left(t_{n}\right)-y_{c} \\
z_{i}\left(t_{n}\right)-z_{c}
\end{array}\right]
$$

ou seja,

$$
\mathrm{U}_{i}\left(t_{n}\right)=\frac{2 \pi}{T}\left[\begin{array}{c}
y_{c}-y_{i}\left(t_{n}\right) \\
x_{i}\left(t_{n}\right)-x_{c} \\
0
\end{array}\right]
$$

Esta velocidade é calculada em todas as células de fronteira e intersecções, durante a simulação; 
- Plano $x z$ : o movimento de rotação no plano $x z$ vai depender do ângulo $\theta_{2 n}$ e da definição dos ângulos:

$$
\theta_{1 n}=-\frac{\pi}{2} \quad \text { e } \quad \varphi_{n}=\frac{\pi}{2} .
$$

Assim, o produto de matrizes de rotação torna-se:

$$
\mathbf{A}\left(\theta_{2 n}\right)=\left[\begin{array}{ccc}
\cos \left(\theta_{2 n}\right) & 0 & -\sin \left(\theta_{2 n}\right) \\
0 & 1 & 0 \\
\sin \left(\theta_{2 n}\right) & 0 & \cos \left(\theta_{2 n}\right)
\end{array}\right]
$$

Portanto, para o plano $x z$ a posição atualizada dos vértices é dada por

$$
\mathbf{P}_{i}\left(t_{n}\right)=\left[\begin{array}{c}
x_{c}+\cos \left(\theta_{2 n}\right)\left(x_{i}\left(t_{n-1}\right)-x_{c}\right)-\sin \left(\theta_{2 n}\right)\left(z_{i}\left(t_{n-1}\right)-z_{c}\right) \\
y_{i}\left(t_{n-1}\right) \\
z_{c}+\sin \left(\theta_{2 n}\right)\left(x_{i}\left(t_{n-1}\right)-x_{c}\right)+\cos \left(\theta_{2 n}\right)\left(z_{i}\left(t_{n-1}\right)-z_{c}\right)
\end{array}\right]
$$

e a velocidade:

$$
\mathrm{U}_{i}\left(t_{n}\right)=\frac{2 \pi}{T}\left[\begin{array}{c}
z_{c}-z_{i}\left(t_{n}\right) \\
0 \\
x_{i}\left(t_{n}\right)-x_{c}
\end{array}\right]
$$

- Plano yz: em relação ao plano $y z$, o movimento de rotação vai depender do ângulo $\theta_{2 n}$, fazendo-se os demais ângulos iguais a zero. Conseqüentemente, as matrizes $\mathbf{R}_{12}^{3}\left(\theta_{1 n}\right)$ e $\mathbf{R}_{12}^{2}\left(\varphi_{n}\right)$ tornam-se matrizes identidade I. Assim, o produto de matrizes de rotação torna-se:

$$
A\left(\theta_{2 n}\right)=\left[\begin{array}{ccc}
1 & 0 & 0 \\
0 & \cos \left(\theta_{2 n}\right) & -\sin \left(\theta_{2 n}\right) \\
0 & \sin \left(\theta_{2 n}\right) & \cos \left(\theta_{2 n}\right)
\end{array}\right]
$$

De acordo com esta matriz, a atualização dos vértices no plano $y z$ é dada por

$$
\mathrm{P}_{i}\left(t_{n}\right)=\left[\begin{array}{c}
x_{i}\left(t_{n-1}\right) \\
y_{c}+\cos \left(\theta_{2 n}\right)\left(y_{i}\left(t_{n-1}\right)-y_{c}\right)-\sin \left(\theta_{2 n}\right)\left(z_{i}\left(t_{n-1}\right)-z_{c}\right) \\
z_{c}+\sin \left(\theta_{2 n}\right)\left(y_{i}\left(t_{n-1}\right)-y_{c}\right)+\cos \left(\theta_{2 n}\right)\left(z_{i}\left(t_{n-1}\right)-z_{c}\right)
\end{array}\right]
$$

e a velocidade é obtida de acordo com:

$$
\mathrm{U}_{i}\left(t_{n}\right)=\frac{2 \pi}{T}\left[\begin{array}{c}
0 \\
z_{c}-z_{i}\left(t_{n}\right) \\
y_{i}\left(t_{n}\right)-y_{c}
\end{array}\right]
$$


O usuário pode escolher qual é o plano de rotação que o objeto vai realizar o movimento e também o tempo inicial (Start Cycle Time) e o tempo final (Stop Cycle Time) do ciclo de rotação, de acordo com a figura 5.21 .

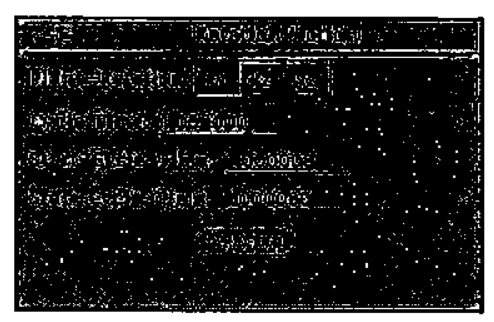

Figura 5.21: Movimento de Rotação

\subsubsection{Movimento Harmônico Simples}

No movimento harmônico simples, o centro de massa do objeto desloca-se sobre a reta que une os pontos $\mathbf{P}_{c_{i}}$ e $\mathbf{P}_{c_{f}}$, analogamente ao caso bidimensional. As coordenadas do ponto $\mathrm{P}_{c_{f}}$ e as constantes relacionadas ao tempo do movimento, podem ser escolhidas pelo usuário, como mostra a figura 5.22.

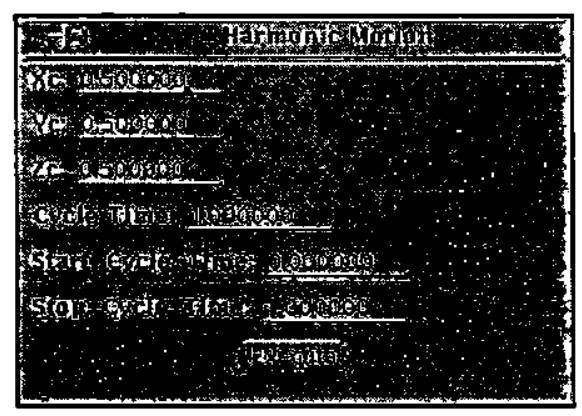

Figura 5.22: Movimento Harmônico Simples

A posição do centro de massa assume a seguinte forma

$$
\mathbf{P}_{c}(t)=\frac{1}{2}\left(\mathbf{P}_{c_{f}}-\mathbf{P}_{c_{i}}\right) \sin \left(-\frac{\pi}{2}+\frac{\pi}{T} t\right)+\frac{1}{2}\left(\mathbf{P}_{c_{i}}+\dot{\mathrm{P}}_{c_{f}}\right)
$$

ou

$$
\mathrm{P}_{c}\left(t_{n}\right)=\left[\begin{array}{c}
\frac{1}{2}\left(x_{c_{f}}-x_{c_{i}}\right) \sin \left(-\frac{\pi}{2}+\frac{\pi}{T} t_{n}\right)+\frac{1}{2}\left(x_{c_{i}}+x_{c_{f}}\right) \\
\frac{1}{2}\left(y_{c_{f}}-y_{c_{i}}\right) \sin \left(-\frac{\pi}{2}+\frac{\pi}{T} t_{n}\right)+\frac{1}{2}\left(y_{c_{i}}+y_{c_{f}}\right) \\
\frac{1}{2}\left(z_{c_{f}^{\prime}}-z_{c_{i}}\right) \sin \left(-\frac{\pi}{2}+\frac{\pi}{T} t_{n}\right)+\frac{1}{2}\left(z_{c_{i}}+z_{c_{f}}\right)
\end{array}\right]
$$


e sua velocidade é definida por

$$
\mathrm{U}_{c}\left(t_{n}\right)=\frac{\pi}{2 T}\left(\mathrm{P}_{c_{f}}-\mathrm{P}_{c_{i}}\right) \cos \left(-\frac{\pi}{2}+\frac{\pi}{T} t_{n}\right)
$$

ou

$$
\mathbf{U}_{c}\left(t_{n}\right)=\left[\begin{array}{c}
\frac{\pi}{2 T}\left(x_{c_{f}}-x_{c_{i}}\right) \cos \left(-\frac{\pi}{2}+\frac{\pi}{T} t_{n}\right) \\
\frac{\pi}{2 T}\left(y_{c_{f}}-y_{c_{i}}\right) \cos \left(-\frac{\pi}{2}+\frac{\pi}{T} t_{n}\right) \\
\frac{\pi}{2 T}\left(z_{c_{f}}-z_{c_{i}}\right) \cos \left(-\frac{\pi}{2}+\frac{\pi}{T} t_{n}\right)
\end{array}\right]
$$

Durante este movimento, o centro de massa, juntamente com os vértices, não realiza o movimento de rotação e portanto, os ângulos $\theta_{1}, \theta_{2}$ e $\varphi$ permanecem constantes.

Assim, o produto de matrizes de rotação torna-se a matriz identidade $I$ e a posição dos vértices é calculada segundo:

$$
\begin{gathered}
\mathbf{P}_{i}\left(t_{n}\right)=\mathbf{P}_{c}\left(t_{n}\right)+\mathbf{P}_{i}\left(t_{n-1}\right)-\mathbf{P}_{c}\left(t_{n-1}\right), \\
\mathbf{P}_{i}\left(t_{n}\right)=\left[\begin{array}{l}
x_{c}\left(t_{n}\right)+x_{i}\left(t_{n-1}\right)-x_{c}\left(t_{n-1}\right) \\
y_{c}\left(t_{n}\right)+y_{i}\left(t_{n-1}\right)-y_{c}\left(t_{n-1}\right) \\
z_{c}\left(t_{n}\right)+z_{i}\left(t_{n-1}\right)-z_{c}\left(t_{n-1}\right)
\end{array}\right] .
\end{gathered}
$$

De maneira análoga ao caso bidimensional, a velocidade dos vértices é igual a velocidade do centro de massa.

Este tipo de movimento está definido para os contêineres e injetores, e a velocidade é calculada em todas as células de fronteira e intersecções destes objetos, no decorrer da simulação.

\subsubsection{Movimento Definido por Interpolação por Splines Cúbica}

O movimento definido pelo usuário, através da interpolação por splines cúbica, é análogo ao caso bidimensional e a posição atualizada do objeto é obtida de acordo com

$$
\mathbf{P}_{i}\left(t_{n}\right)=\mathbf{P}_{c}\left(t_{n}\right)+\mathbf{A}\left(\theta_{1 n}, \theta_{2 n}, \varphi_{n}\right)\left(\mathbf{P}_{i}\left(t_{n-1}\right)-\mathbf{P}_{c}\left(t_{n-1}\right)\right)
$$

onde

$$
\mathrm{P}_{c}\left(t_{n}\right)=S_{\mathrm{c}}\left(t_{n}\right)
$$

$\mathrm{e}$

$$
\theta_{1 n}=S_{\theta_{1}}\left(t_{n}\right)-S_{\theta_{1}}\left(t_{n-1}\right)
$$




$$
\begin{gathered}
\theta_{2 n}=S_{\theta_{2}}\left(t_{n}\right)-S_{\theta_{2}}\left(t_{n-1}\right), \\
\varphi_{n}=S_{\varphi}\left(t_{n}\right)-S_{\varphi}\left(t_{n-1}\right) .
\end{gathered}
$$

A velocidade dos vértices é calculada segundo

$$
\mathrm{U}_{i}\left(t_{n}\right)=\mathbf{P}_{c}^{\prime}\left(t_{n}\right)+\mathrm{A}^{\prime}\left(\theta_{1 n}, \theta_{2 n}, \varphi_{n}\right) \theta_{1}^{\prime}\left(t_{n}\right) \theta_{2}^{\prime}\left(t_{n}\right) \varphi^{\prime}\left(t_{n}\right) \mathrm{A}^{-1}\left(\theta_{1 n}, \theta_{2 n}, \varphi_{n}\right)\left(\mathbf{P}_{i}\left(t_{n}\right)-\mathrm{P}_{c}\left(t_{n}\right)\right)
$$

com

$$
\mathrm{P}_{c}^{\prime}\left(t_{n}\right)=S_{c}^{\prime}\left(t_{n}\right)
$$

e

$$
\begin{aligned}
\theta_{1}^{\prime} & =S_{\theta_{1}}^{\prime}\left(t_{n}\right), \\
\theta_{2}^{\prime} & =S_{\theta_{2}}^{\prime}\left(t_{n}\right), \\
\varphi^{\prime} & =S_{\varphi}^{\prime}\left(t_{n}\right) .
\end{aligned}
$$

A seguir, apresenta-se um exemplo que ilustra um tipo de movimento definido pelo usuário.

Considera-se um sistema de coordenadas cartesianas de $t$ em função de $x_{c}$. Obtémse, por exemplo, os pontos de interpolação para a coordenada $x_{c}$ do centro de massa, conforme mostrà a figura 5.23 .
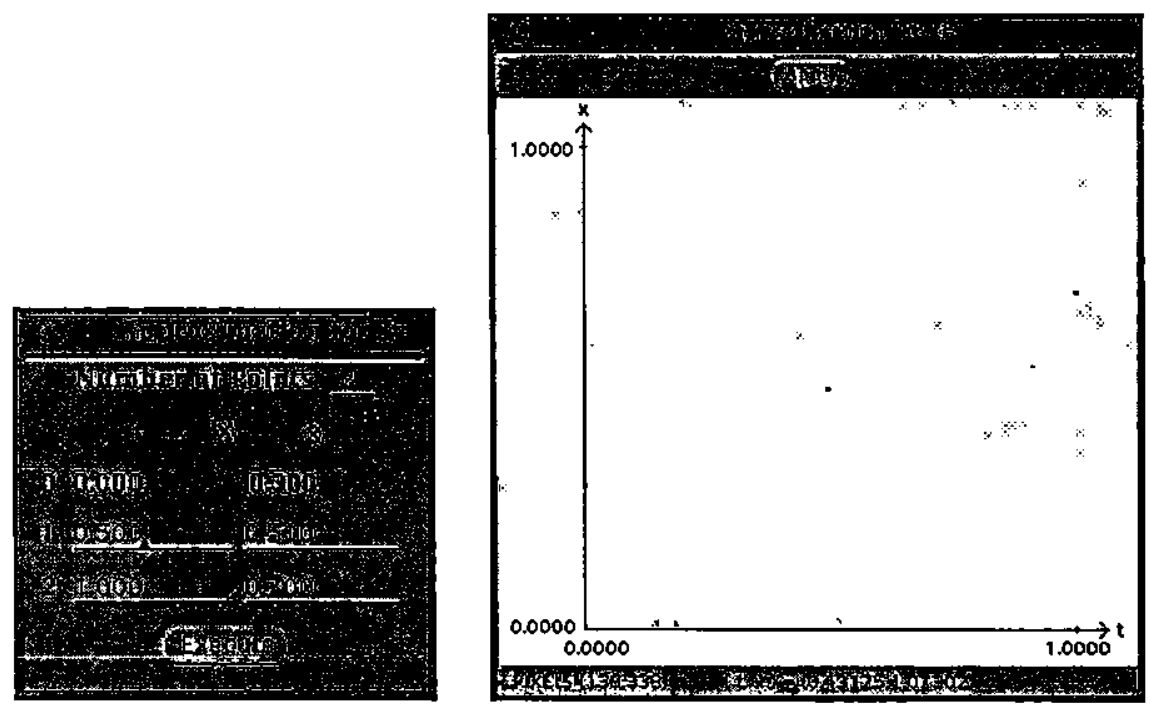

Figura 5.23: Interpolação por splines cúbica para $x_{c}$

Assim, a aproximação pela função splines cúbica para a coordenada $x_{c}$ torna-se: 


$$
\begin{gathered}
x_{c}(t)=\frac{1}{h_{i}^{2}}\left[\left(t-t_{i+1}\right)^{2}\left(x_{c i}+\left(t-t_{i}\right)\left(\frac{2 x_{c i}}{h_{i}}+x_{c i}^{\prime}\right)\right)+\right. \\
\left.\left(t-t_{i}\right)^{2}\left(x_{c i+1}+\left(t-t_{i+1}\right)\left(x_{c i+1}^{\prime}-\frac{2 x_{c i+1}}{h_{i}}\right)\right)\right] .
\end{gathered}
$$

Considera-se agora o mesmo sistema de coordenadas cartesianas para as coordenadas $y_{c}$ e $z_{c}$ e suponha-se que o usuário escolha os pontos de interpolação, de acordo com as figuras 5.24 e 5.25 .
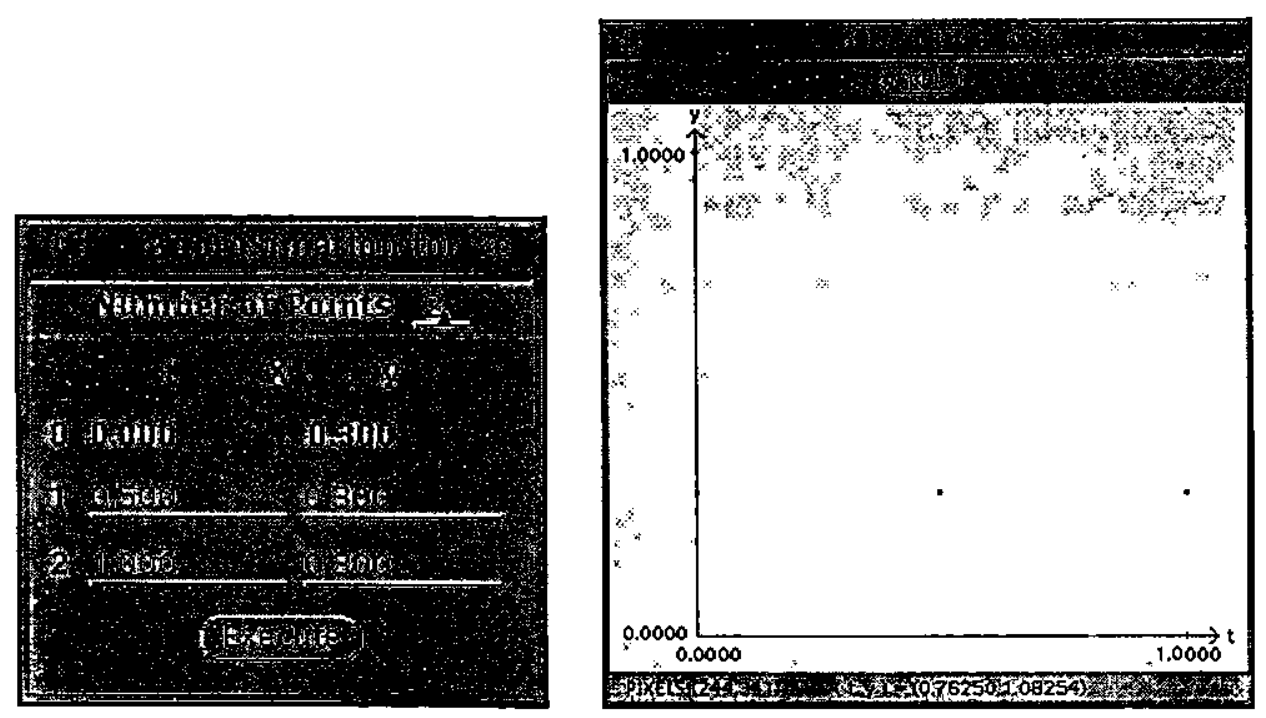

Figura 5.24: Interpolação por splines cúbica para $y_{c}$
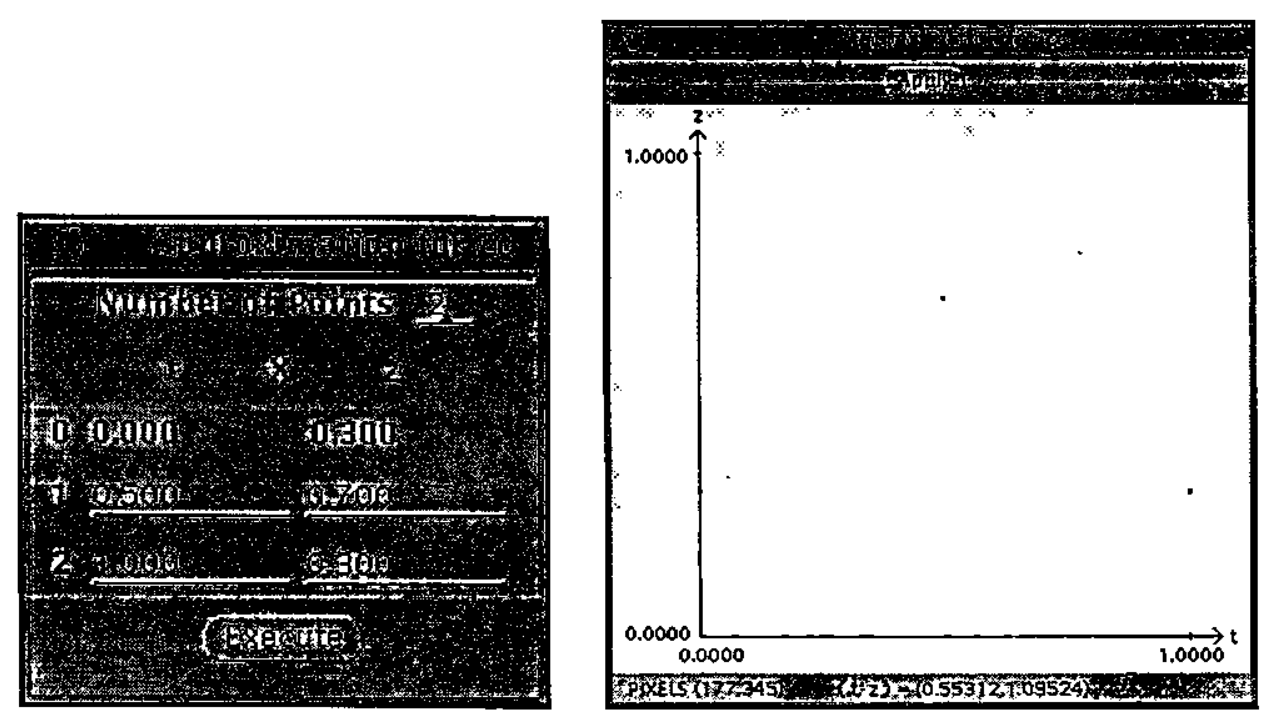

Figura 5.25: Interpolação por splines cúbica para $z_{c}$ 
Assim,

$$
\begin{gathered}
y_{c}(t)=\frac{1}{h_{i}^{2}}\left[\left(t-t_{i+1}\right)^{2}\left(y_{c_{i}}+\left(t-t_{i}\right)\left(\frac{2 y_{c_{i}}}{h_{i}}+y_{c i}^{\prime}\right)\right)+\right. \\
\left.\left(t-t_{i}\right)^{2}\left(y_{c_{i+1}}+\left(t-t_{i+1}\right)\left(y_{c i+1}^{\prime}-\frac{2 y_{c_{i+1}}}{h_{i}}\right)\right)\right] \\
z_{c}(t)=\frac{1}{h_{i}^{2}}\left[\left(t-t_{i+1}\right)^{2}\left(z_{c i}+\left(t-t_{i}\right)\left(\frac{2 z_{c i}}{h_{i}}+z_{c i}^{\prime}\right)\right)+\right. \\
\left.\left(t-t_{i}\right)^{2}\left(z_{c i+1}+\left(t-t_{i+1}\right)\left(z_{c i+1}^{\prime}-\frac{2 z_{c i+1}}{h_{i}}\right)\right)\right] .
\end{gathered}
$$

Tem-se que as coordenadas do centro de massa podem ser escritas de acordo com as equações $5.55,5.56$ e 5.57 .

A velocidade do centro de massa é obtida pela derivação de cada termo de suas coordenadas em relação ao tempo. Para a coordenada $x_{c}$ obtém-se:

$$
\begin{aligned}
x_{c}^{\prime}(t)=\frac{1}{h_{i}^{2}} & {\left[\left(t-t_{i+1}\right)^{2}\left(\frac{2 x_{c i}}{h_{i}}+x_{c i}^{\prime}\right)+\left(t-t_{i}\right)^{2}\left(x_{c i+1}^{\prime}-\frac{2 x_{c i+1}}{h_{i}}\right)+\right.} \\
& 2\left(t-t_{i+1}\right)\left(x_{c i}+\frac{2 x_{c i}}{h_{i}}\left(t-t_{i}\right)\left(x_{c i}-x_{c i+1}\right)\right)+ \\
& \left.2\left(t-t_{i}\right)\left(x_{c i+1}+\left(t-t_{i+1}\right)\left(x_{c i}^{\prime}+x_{c i+1}^{\prime}\right)\right)\right]
\end{aligned}
$$

Analogamente, para a coordenada $y_{c}$ e $z_{c}$ do centro de massa:

$$
\begin{aligned}
y_{c}^{\prime}(t)=\frac{1}{h_{i}^{2}} & {\left[\left(t-t_{i+1}\right)^{2}\left(\frac{2 y_{c i}}{h_{i}}+y_{c i}^{\prime}\right)+\left(t-t_{i}\right)^{2}\left(y_{c i+1}^{\prime}-\frac{2 y_{c i+1}}{h_{i}}\right)+\right.} \\
2\left(t-t_{i+1}\right)\left(y_{c i}+\frac{2 y_{c i}}{h_{i}}\left(t-t_{i}\right)\left(y_{c i}-y_{c i+1}\right)\right)+ & \\
& \left.2\left(t-t_{i}\right)\left(y_{c i+1}+\left(t-t_{i+1}\right)\left(y_{c i}^{\prime}+y_{c i+1}^{\prime}\right)\right)\right], \\
z_{c}^{\prime}(t)=\frac{1}{h_{i}^{2}} & {\left[\left(t-t_{i+1}\right)^{2}\left(\frac{2 z_{c i}}{h_{i}}+z_{c i}^{\prime}\right)+\left(t-t_{i}\right)^{2}\left(z_{c i+1}^{\prime}-\frac{2 z_{c i+1}}{h_{i}}\right)+\right.} \\
& 2\left(t-t_{i+1}\right)\left(z_{c i}+\frac{2 z_{c i}}{h_{i}}\left(t-t_{i}\right)\left(z_{c i}-z_{c i+1}\right)\right)+ \\
& \left.2\left(t-t_{i}\right)\left(z_{c i+1}+\left(t-t_{i+1}\right)\left(z_{c i}^{\prime}+z_{c i+1}^{\prime}\right)\right)\right] .
\end{aligned}
$$

Para a aproximação dos ângulos $\theta_{1}, \theta_{2}$ e $\varphi$, o usuário poderá escolher apenas um ponto de interpolação, como mostra a figura 5.26 . 

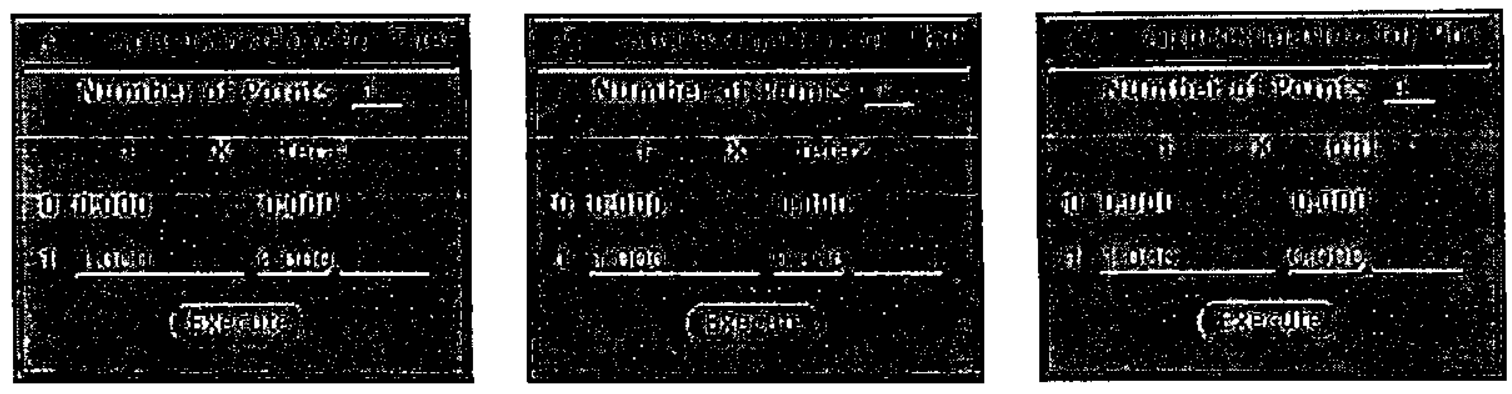

Figura 5.26: Interpolação por splines cúbica para os ângulos de rotação

De acordo com os pontos de interpolação, pode-se notar que os ângulos $\theta_{1}, \theta_{2}$ e $\varphi$ permanecem constantes para qualquer instante de tempo.

Este tipo de movimento definido pelo usuário é um caso particular de movimento de translação no plano $x z$, em que a orientação do objeto não sofre alteraçōes durante a simulação. Deste modo, a posição atualizada dos vértices é definida de acordo com a equação 5.38 , ou seja

$$
P_{i}\left(t_{n}\right)=\left[\begin{array}{c}
x_{c}\left(t_{n}\right)+x_{i}\left(t_{n-1}\right)-x_{c}\left(t_{n-1}\right) \\
y_{c}\left(t_{n}\right)+y_{i}\left(t_{n-1}\right)-y_{c}\left(t_{n-1}\right) \\
z_{c}\left(t_{n}\right)+z_{i}\left(t_{n-1}\right)-z_{c}\left(t_{n-1}\right)
\end{array}\right]
$$

e a velocidade torna-se igual a velocidade do centro de massa. 


\section{Capítulo 6}

\section{Exemplos Numéricos}

\subsection{Considerações Iniciais}

Este capítulo apresenta simulações no modelador de movimentos bi e tridimensional. Trata-se de escoamentos do tipo Newtoniano, com condição de contorno no slip.

\subsection{Exemplos Bidimensionais}

\section{Exemplo 1}

Este modelo de simulação possui dois contêineres do tipo Box, sendo que um deles, inicialmente, contém fluido do tipo Cube. Os dados que configuram o escoamento são:

- Dimensões do domínio: $0.05 \times 0.05$;

- Tamanho da malha: $100 \times 100$ células;

- Tempo inicial: 0.0, tempo final: 0.20 ;

- Valor de referência para comprimento: 0.02;

- Valor de referência para velocidade: 0.5 ;

- Viscosidade: 0.0005;

- Número de Reynolds: 20.0;

- Número de Frouden: 0.885889;

- Número de Strouhal: 25.0;

- Tipo de Movimento: Rotação;

- Tempo do ciclo: 0.2;

- Tempo de parada do ciclo de rotação: 0.075 . 
Durante esta simulação o contêiner com fluido vai realizar movimento de rotação, derramando o fluido sobre o contêiner vazio.

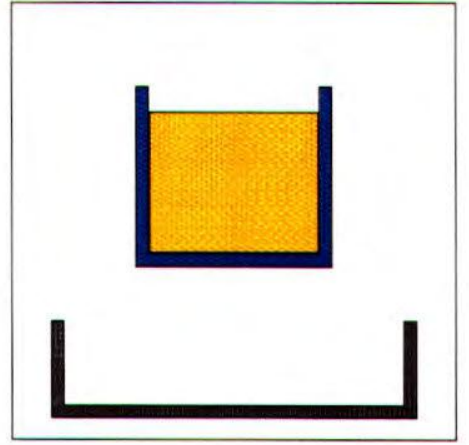

$t=0.0$

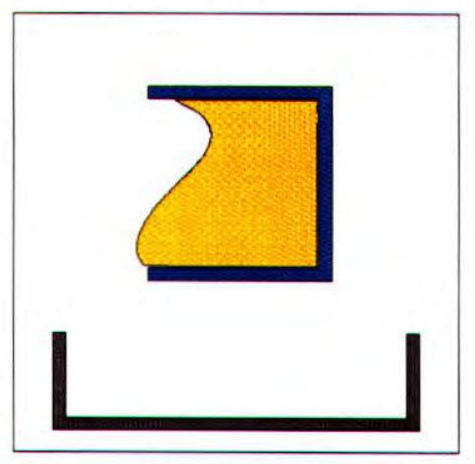

$t=0.05$

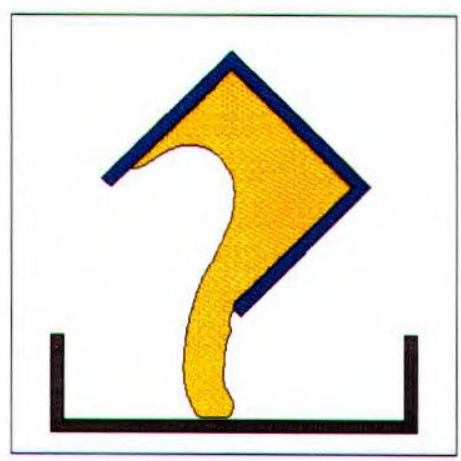

$t=0.09$

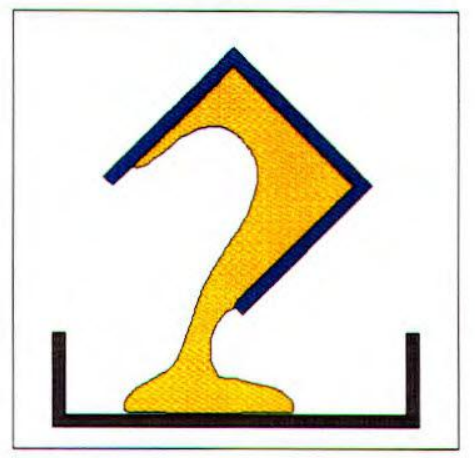

$t=0.14$

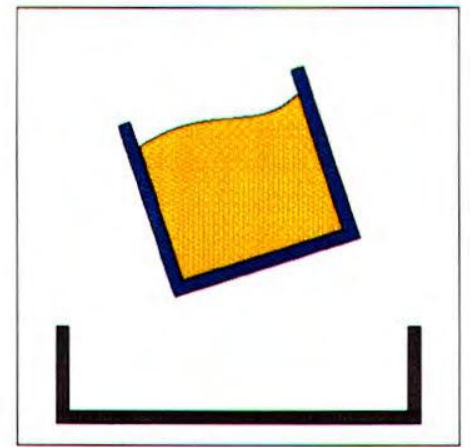

$t=0.01$

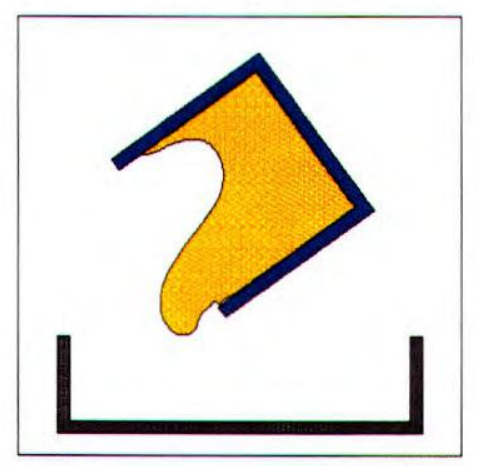

$t=0.07$

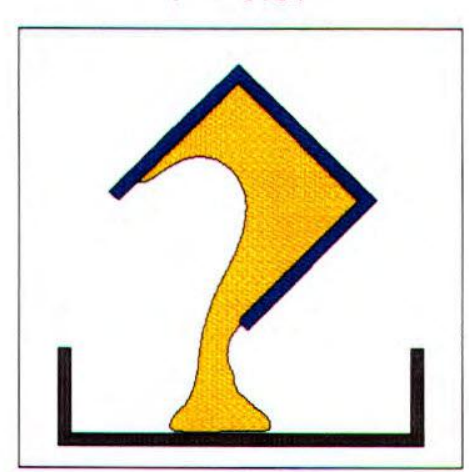

$t=0.10$

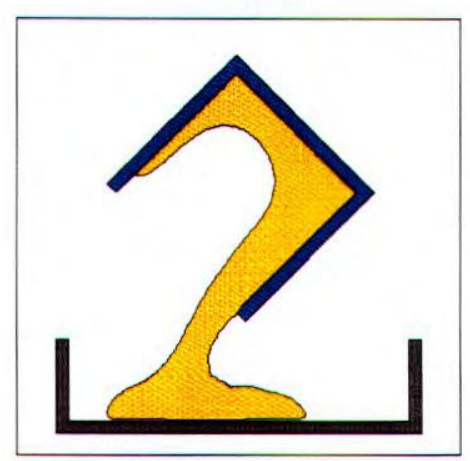

$t=0.17$

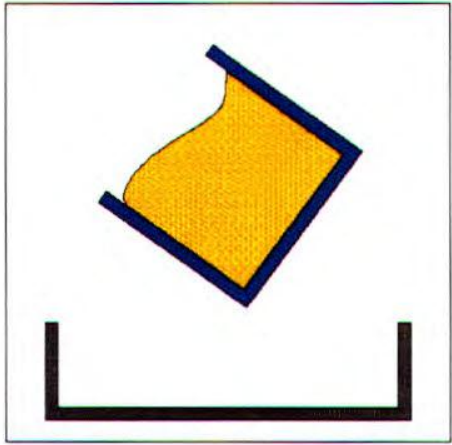

$t=0.03$

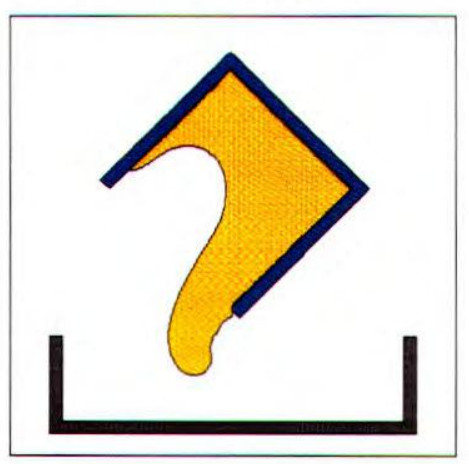

$t=0.08$

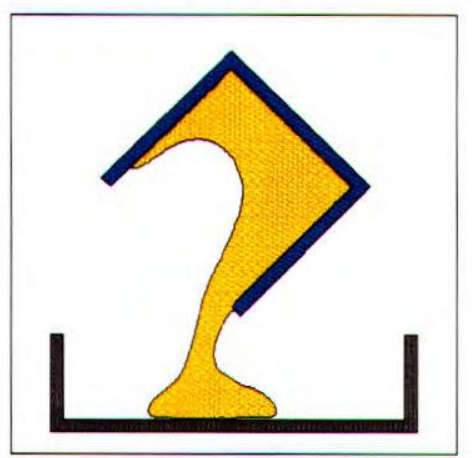

$t=0.11$

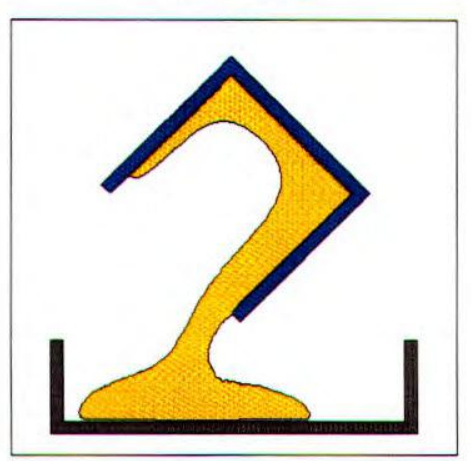

$t=0.2$ 


\section{Exemplo 2}

Este modelo de simulaçāo possui um contêiner do tipo Box-Cilinder contendo um fluido do tipo Sphere. Os dados que configuram o escoamento são:

- Dimensões do domínio: $0.05 \times 0.03$;

- Tamanho da malha: $50 \times 30$ células;

- Tempo inicial: 0.0, tempo final: 0.20;

- Valor de referência para comprimento: 0.016;

- Valor de referência para velocidade: 1.0;

- Viscosidade: 0.0008;

- Número de Reynolds: 20.0;

- Número de Frouden: 0.396182;

- Número de Strouhal: 62.50;

- Tipo de Movimento: Harmônico simples;

- Coordenada $x_{c}$ do Centro de Massa Inicial: 0.0110

- Coordenada $y_{c}$ do Centro de Massa Inicial: 0.009852

- Coordenada $x_{f}$ do Centro de Massa Final: 0.039000

- Coordenada $y_{f}$ do Centro de Massa Final: 0.009852

- Tempo do Ciclo: 0.10

Em linhas gerais, o movimento harmônico simples pode ser descrito da seguinte maneira: em $t=0.0$ o centro de massa do objeto tem coordenadas $\left(x_{c}, y_{c}\right)$. Durante a simulação o objeto move-se para o lado direito, ganhando velocidade até atingir $\mathrm{t}=$ 0.05. O objeto passa entāo a diminuir a velocidade até atingir $t=0.10$. A direção do movimento é aí revertida, e o objeto se move para o lado esquerdo, ganhando velocidade até atingir $\mathrm{t}=0.15$. Logo após, perde velocidade até retornar à posição inicial em $\mathrm{t}=$ 0.20 . 


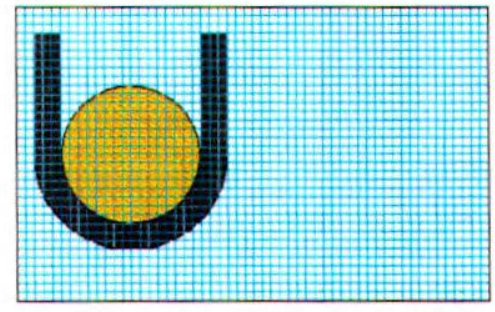

$$
t=0.0
$$

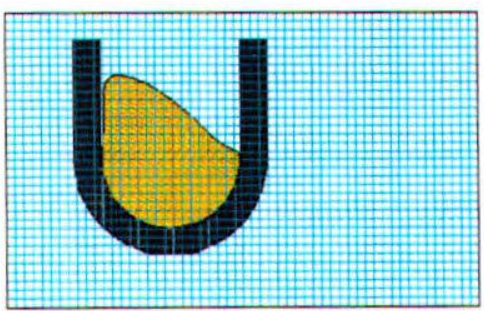

$$
t=0.03
$$

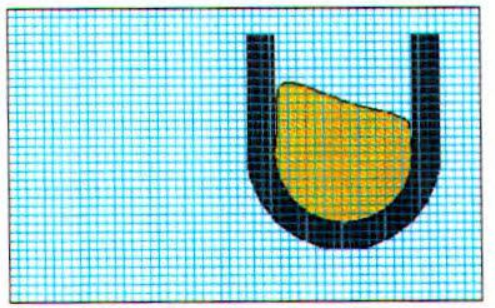

$$
t=0.08
$$

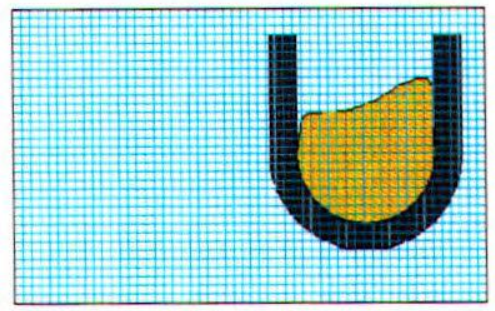

$t=0.11$

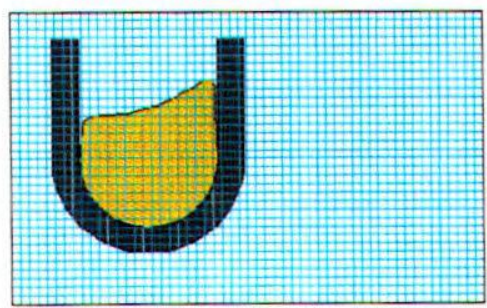

$$
t=0.18
$$

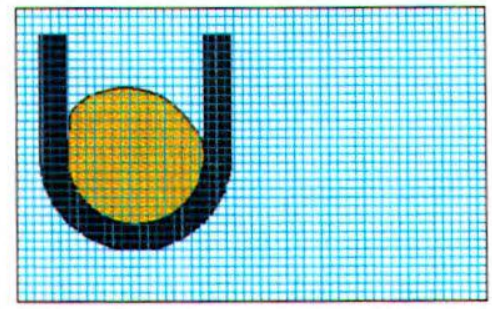

$t=0.01$

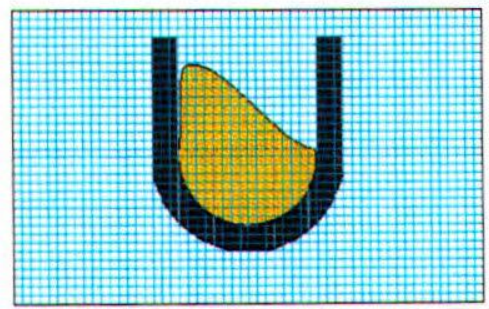

$t=0.05$

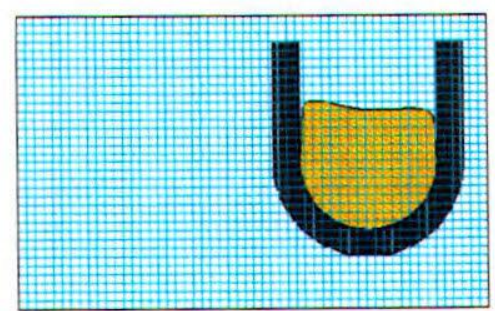

$t=0.09$

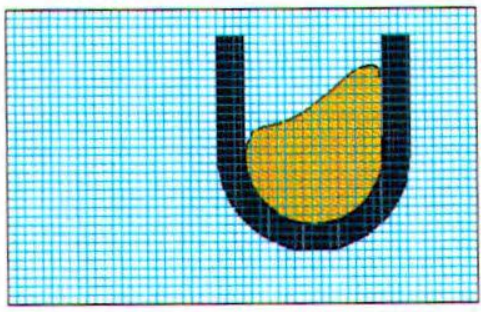

$t=0.13$

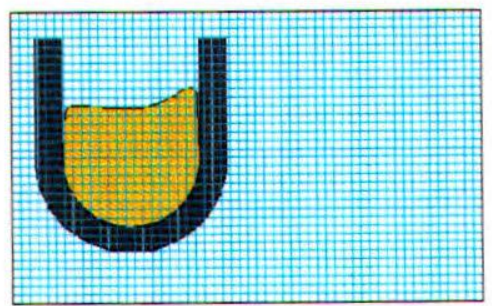

$t=0.19$

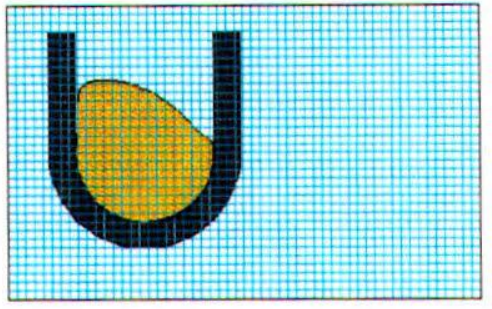

$t=0.02$

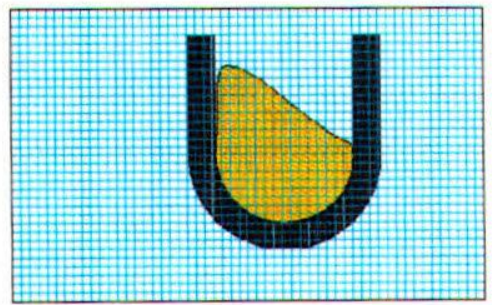

$t=0.06$

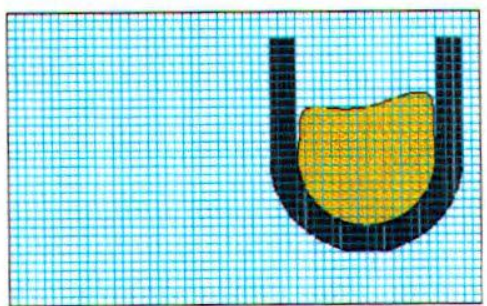

$$
t=0.10
$$

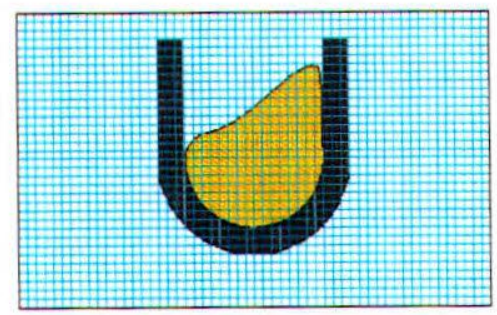

$$
t=0.15
$$

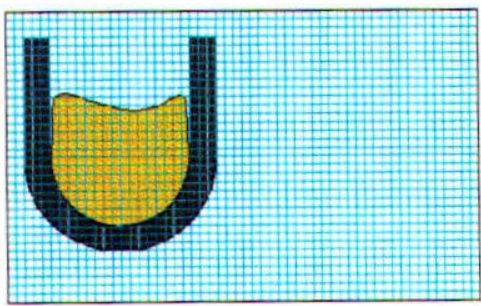

$$
t=0.20
$$




\section{Exemplo 3}

Este modelo de simulação possui um injetor do tipo Cube e um contêiner do tipo Box inclinado. Os dados que configuram o escoamento são:

- Dimensões do domínio: $0.05 \times 0.05$;

- Tamanho da malha: $100 \times 100$ células;

- Tempo inicial: 0.0, tempo final: 0.20;

- Valor de referência para comprimento: 0.004;

- Valor de referência para velocidade: 1.0

- Viscosidade: 0.004

- Número de Reynolds: 1.0 ;

- Número de Frouden: 0.198091;

- Número de Strouhal: 250.0;

- Tipo de Movimento: Harmônico simples;

- Coordenada $x_{c}$ do Centro de Massa Inicial: 0.0250

- Coordenada $y_{c}$ do Centro de Massa Inicial: 0.04750

- Coordenada $x_{f}$ do Centro de Massa Final: 0.0250

- Coordenada $y_{f}$ do Centro de Massa Final: 0.0380

- Tempo do Ciclo: 0.10

$O$ injetor desloca-se ao longo do eixo $y$. Até $o$ instante $t=0.04$ o injetor move-se para baixo sem injeção de fluido. A partir deste instante, o movimento continua com injeção de fluido e é revertido no instante $t=0.10$. Então, o injetor move-se para cima até atingir $o$ instante $t=0.20$. 


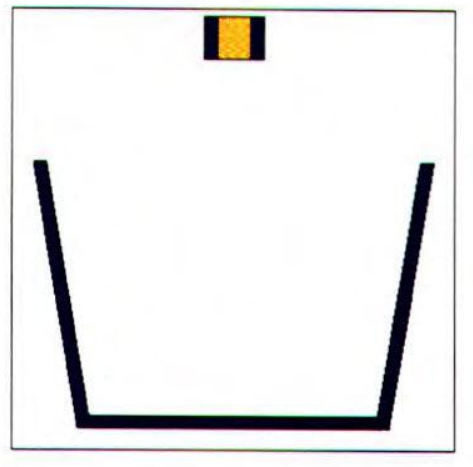

$t=0.0$

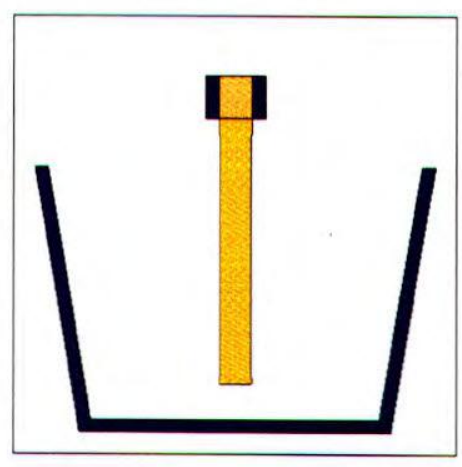

$t=0.06$

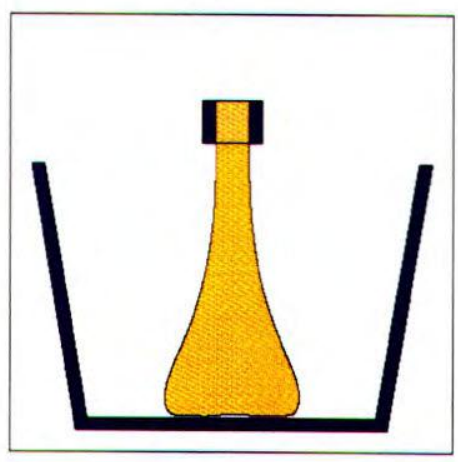

$t=0.10$

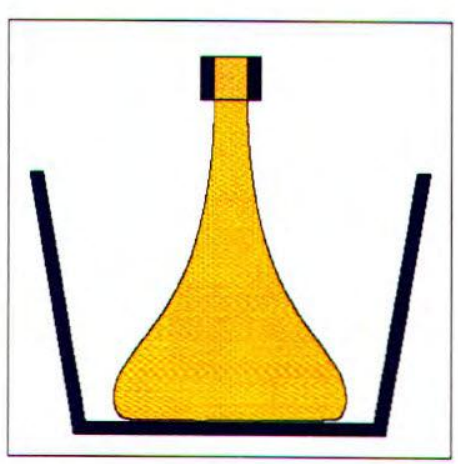

$t=0.16$

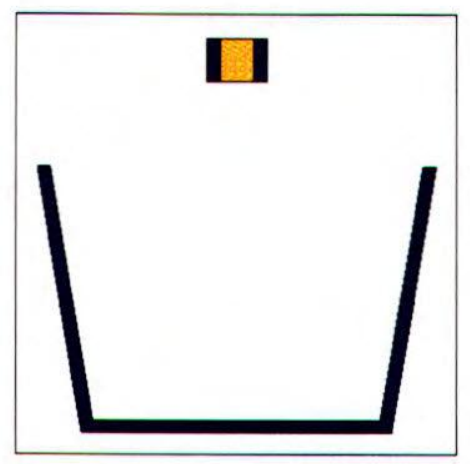

$t=0.03$

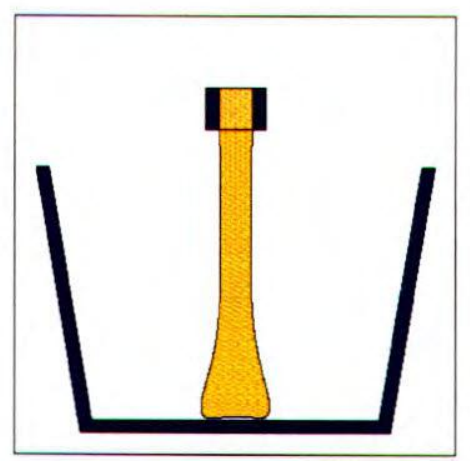

$t=0.07$

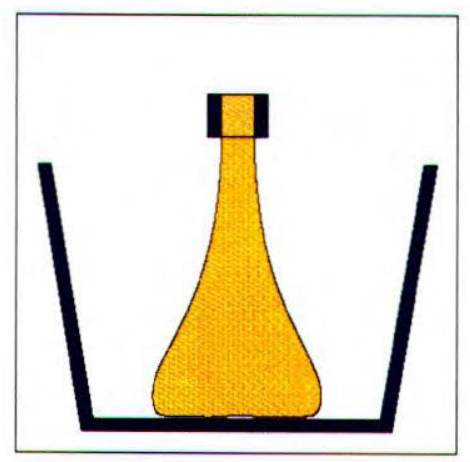

$t=0.12$

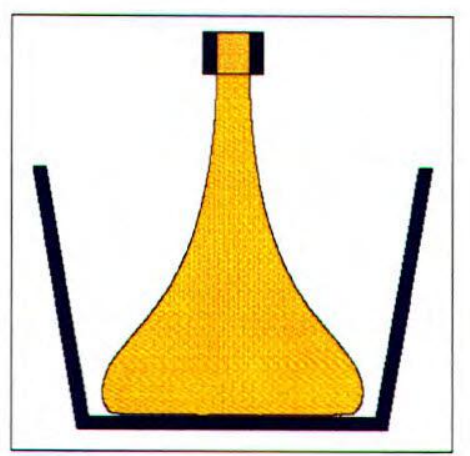

$t=0.18$

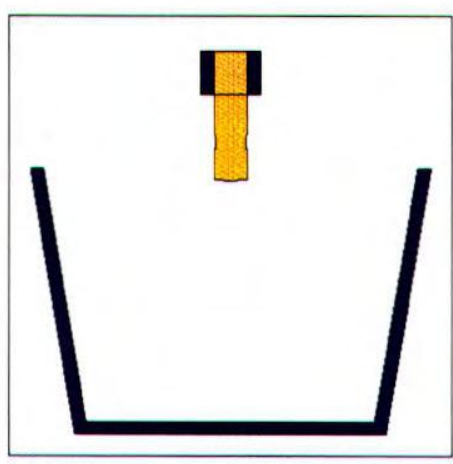

$t=0.04$

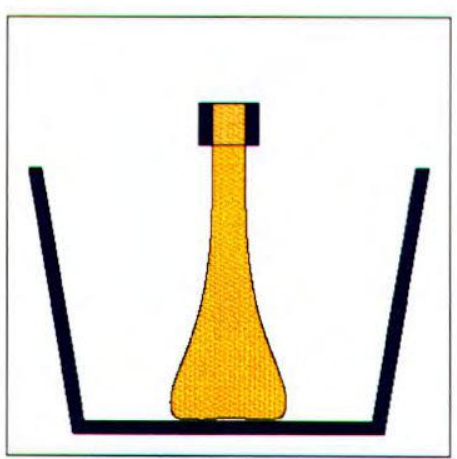

$t=0.09$

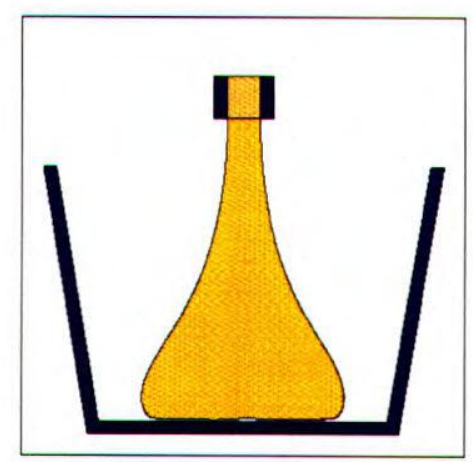

$t=0.14$

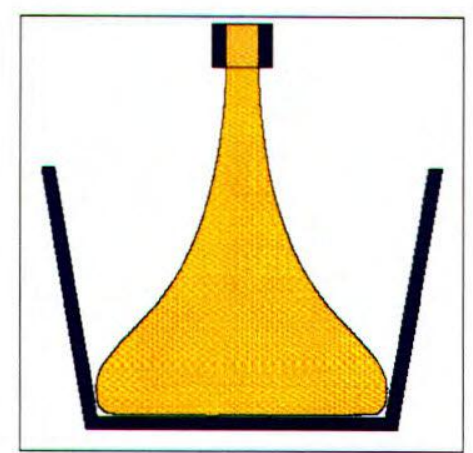

$t=0.20$ 


\section{Exemplo 4}

Este modelo de simulação possui um contêiner do tipo Box, que contém um fluido do tipo Cube, e um contêiner do tipo Sphere. Os dados que configuram o escoamento são:

- Dimensões do domínio: 0.05×0.05;

- Tamanho da malha: $100 \times 100$ células;

- Tempo inicial: 0.0, tempo final: 0.40;

- Valor de referência para comprimento: 0.04;

- Valor de referência para velocidade: 0.25;

- Viscosidade: 0.0004;

- Número de Reynolds: 25.0;

- Número de Frouden: 2.505674;

- Número de Strouhal: 6.250;

- Tipo de Movimento: Harmônico simples;

- Coordenada $x_{c}$ do Centro de Massa Inicial: 0.0250

- Coordenada $y_{c}$ do Centro de Massa Inicial: 0.0440

- Coordenada $x_{f}$ do Centro de Massa Final: 0.0250

- Coordenada $y_{f}$ do Centro de Massa Final: 0.0105

- Tempo do ciclo: 0.10

- Tempo de parada do ciclo: 0.10

Durante esta simulação o contêiner do tipo Sphere vai realizar o movimento harmônico simples, mergulhando no fluido até atingir $t=0.10$. Em seguida, o objeto permanece em posiçāo constante até o final de simulação e o fluido cobre o objeto até atingir o estado estacionário em $t=0.40$. 


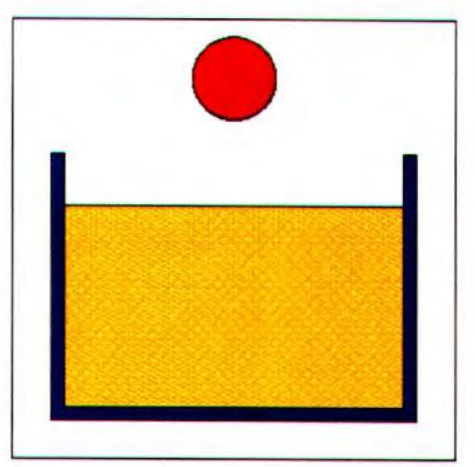

$t=0.0$

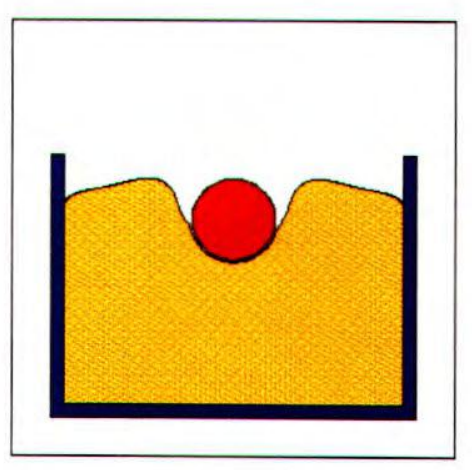

$t=0.05$

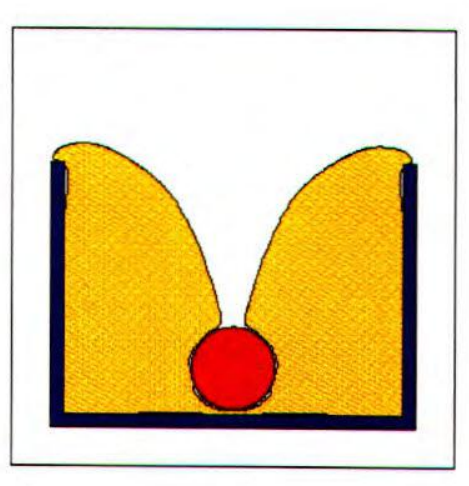

$t=0.12$

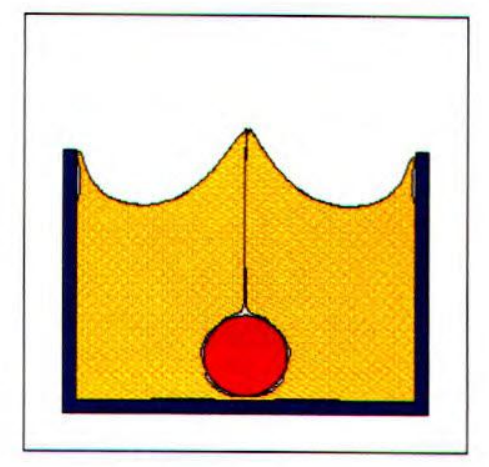

$t=0.18$

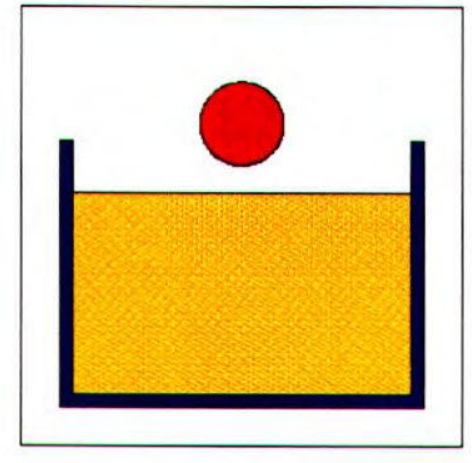

$t=0.03$

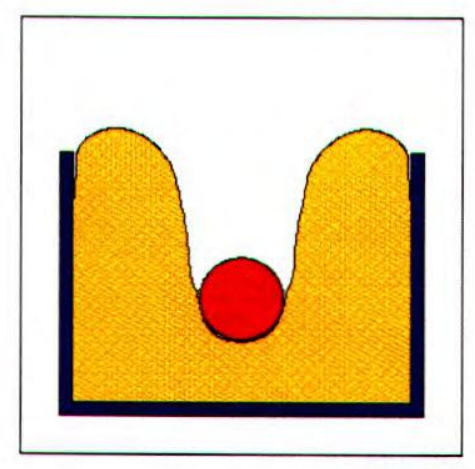

$t=0.07$

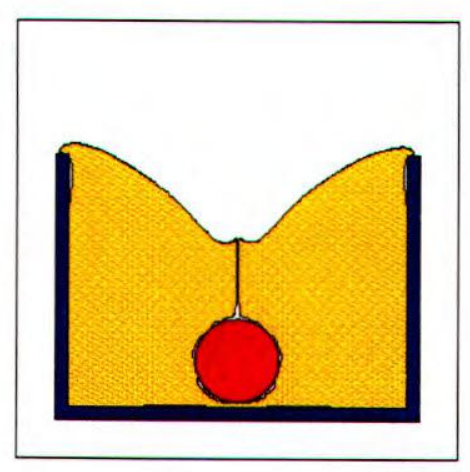

$t=0.13$

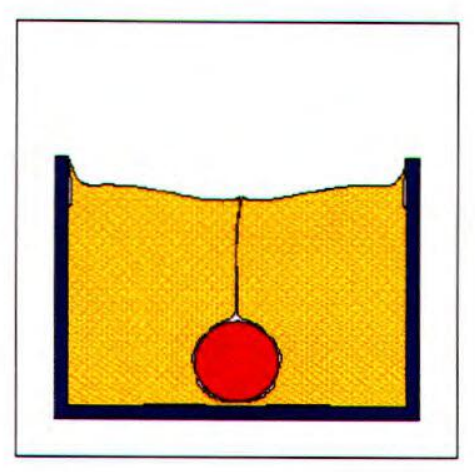

$t=0.28$

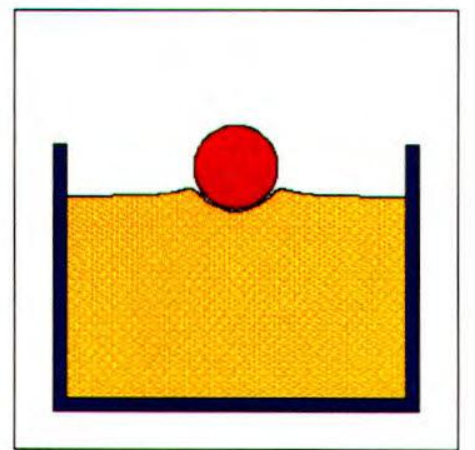

$t=0.04$

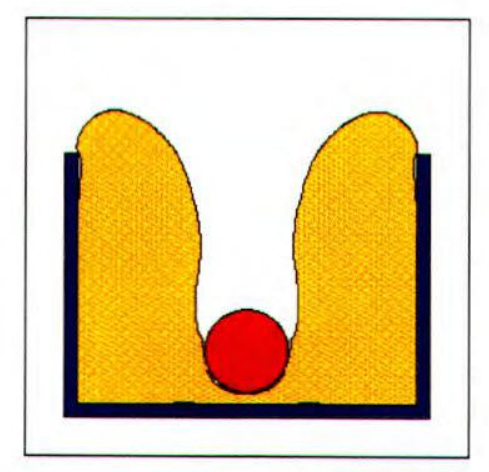

$t=0.09$

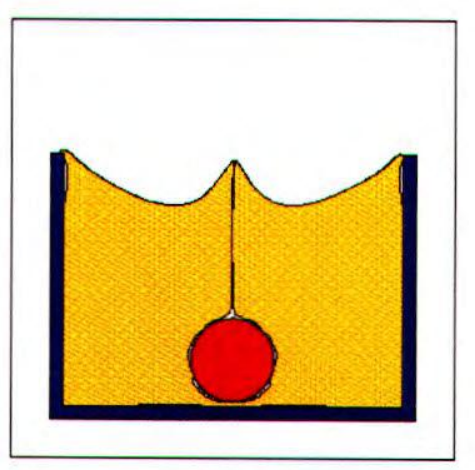

$t=0.15$

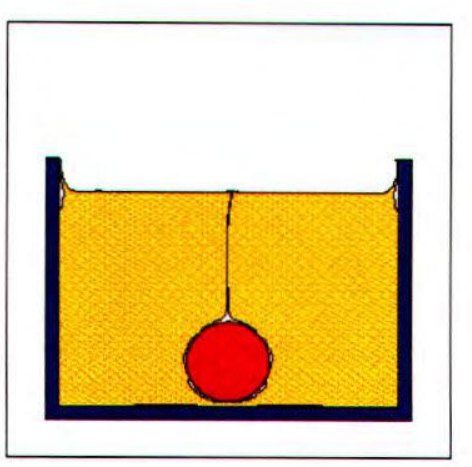

$t=0.40$ 


\section{Exemplo 5}

Este modelo de simulação possui um contêiner do tipo Box contendo um fluido do tipo Cube. Os dados que configuram o escoamento são:

- Dimensões do domínio: 0.05×0.05;

- Tamanho da malha: 50×50 células;

- Tempo inicial: 0.0, tempo final: 0.20 ;

- Valor de referência para comprimento: 0.02;

- Valor de referência para velocidade: 1.0 ;

- Viscosidade: 0.001;

- Número de Reynolds: 20.0;

- Número de Frouden: 0.442945;

- Número de Strouhal: 50.0;

- Tipo de Movimento: Aproximação por splines cúbica;

- Pontos escolhidos para a aproximação:

- Coordenada x do centro de massa

$\mathrm{t}=0.00, x_{c}=0.0130$

$\mathrm{t}=0.05, x_{c}=0.0250$

$\mathrm{t}=0.10, x_{c}=0.0370$

$\mathrm{t}=0.15, x_{c}=0.0250$

$\mathrm{t}=0.20, x_{c}=0.0130$

- Coordenada y do centro de massa

$\mathrm{t}=0.00, y_{c}=0.024750$

$\mathrm{t}=0.05, y_{c}=0.034750$

$\mathrm{t}=0.10, y_{c}=0.024750$

$\mathrm{t}=0.15, y_{c}=0.014750$

$\mathrm{t}=0.20, y_{c}=0.024750$

- Ângulo de Orientação

$\mathrm{t}=0.00, \theta=0.0$

$\mathrm{t}=0.20, \theta=0.0$

No decorrer desta simulaçāo o objeto percorre uma trajetória aproximada pela splines cúbica de acordo com os pontos de interpolaçāo. 


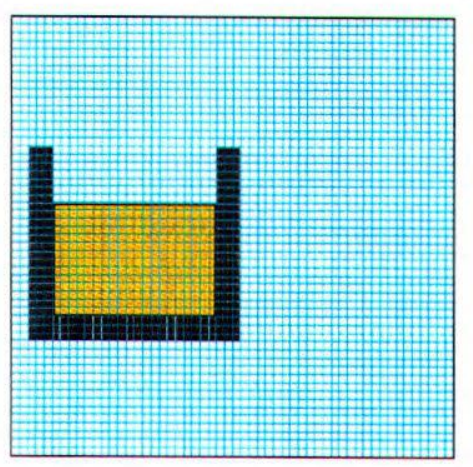

$t=0.0$

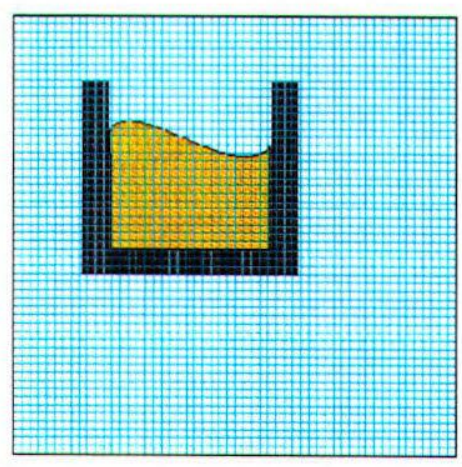

$t=0.03$

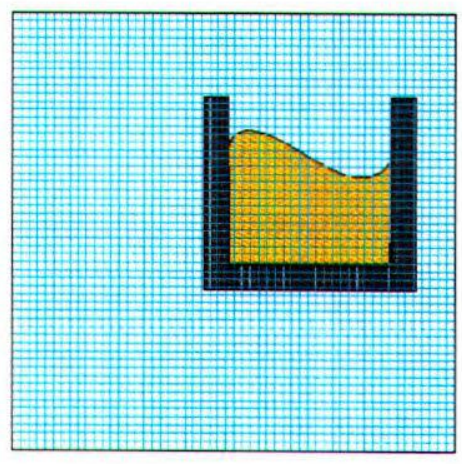

$t=0.08$

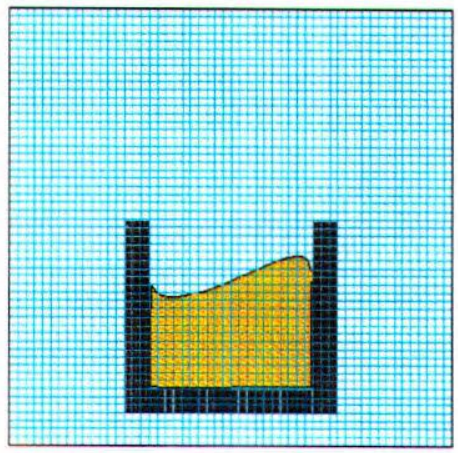

$t=0.15$

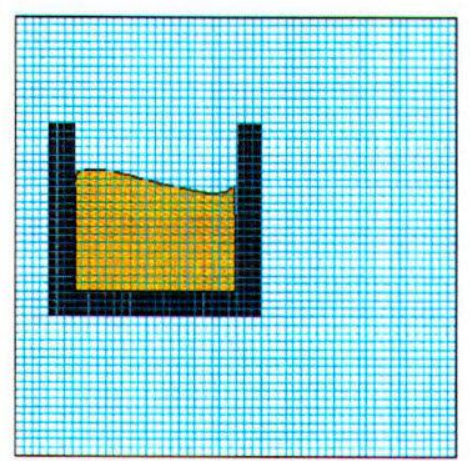

$t=0.01$

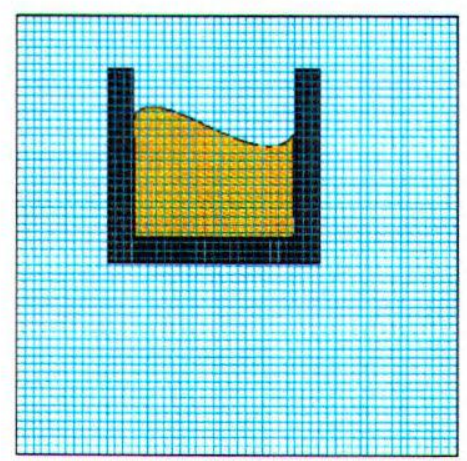

$t=0.04$

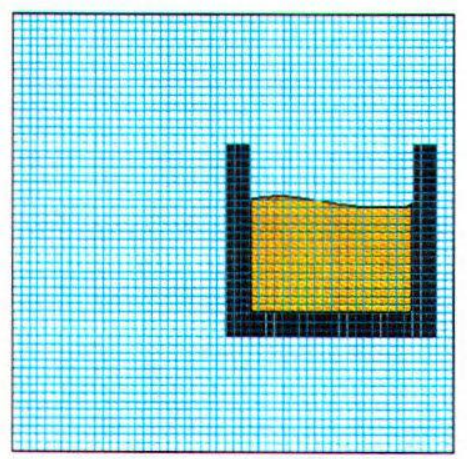

$t=0.10$

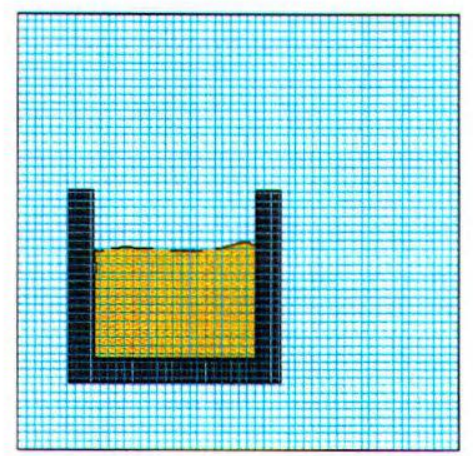

$t=0.18$

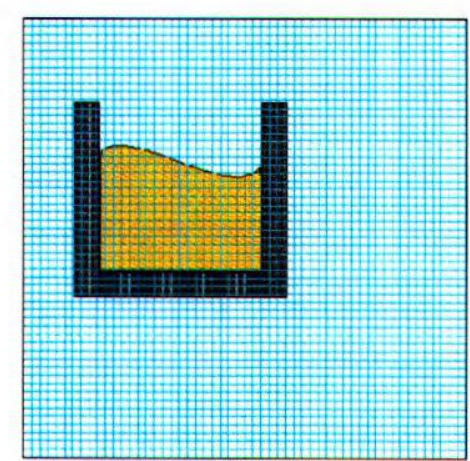

$$
t=0.02
$$

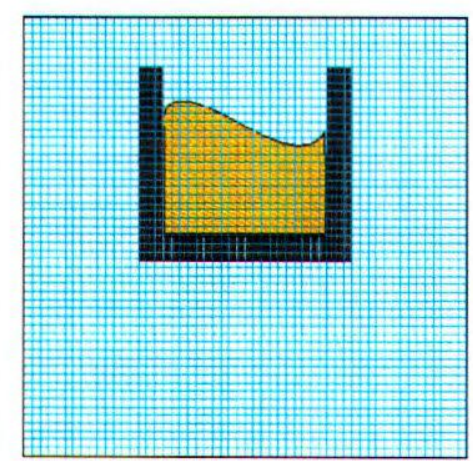

$t=0.05$

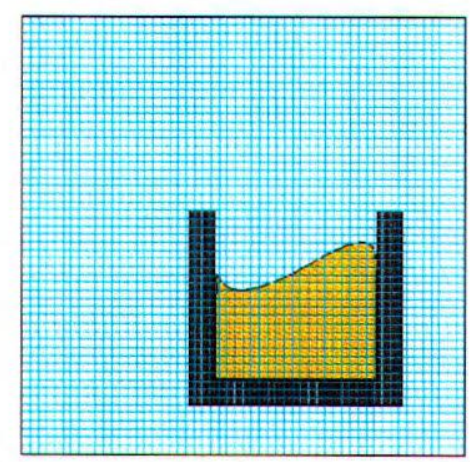

$t=0.13$

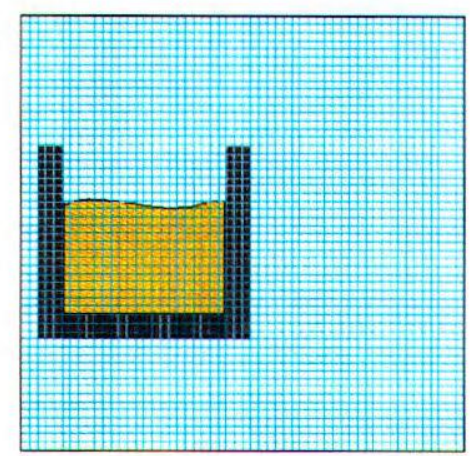

$$
t=0.20
$$




\subsection{Exemplos Tridimensionais}

\section{Exemplo 1}

Este modelo de simulação possui um contêiner do tipo Box, contendo um fluido do tipo Cube, e um contêiner do tipo Cube, como mostra a figura 6.1. Os dados que configuram o escoamento são:

- Dimensões do domínio: $0.02 \times 0.02 \times 0.02$;

- Tamanho da malha: $20 \times 20 \times 20$ células;

- Valor de referência para comprimento: 0.014;

- Valor de referência para velocidade: 1.0 ;

- Viscosidade: 0.0014;

- Número de Reynolds: 10.0;

- Número de Frouden: 2.69837;

- Número de Strouhal: 71.428571;

- Tipo de Movimento: Rotação.

- Tempo do ciclo de rotação : 0.003

O contêiner do tipo Cube, na cor vermelha, vai realizar o movimento de rotação no plano $x z$, misturando o fluido durante a simulação.

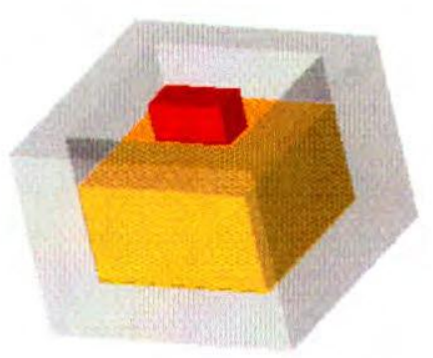

Figura 6.1: Objeto no instante $t=0.0$ 


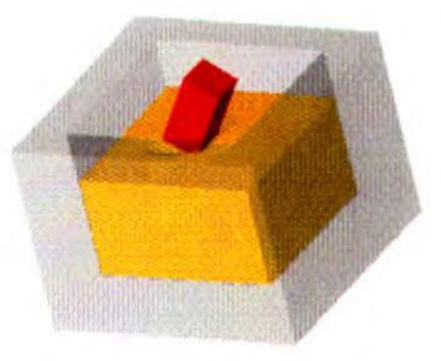

$t=0.0005$

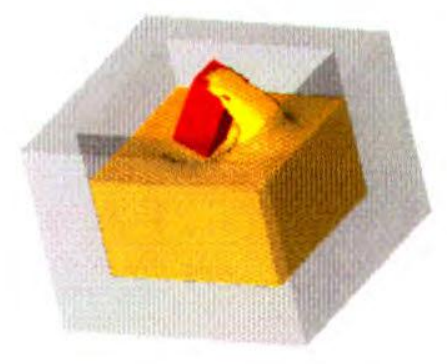

$t=0.002$

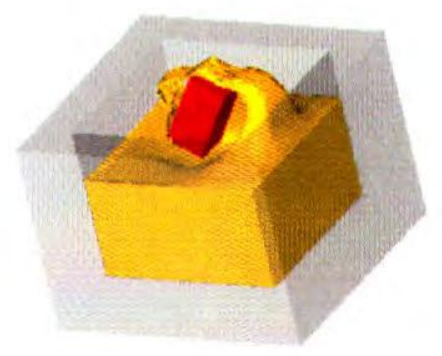

$t=0.0035$

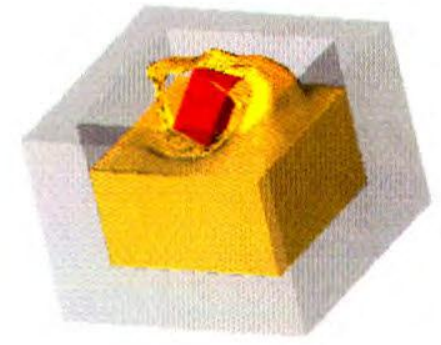

$t=0.005$

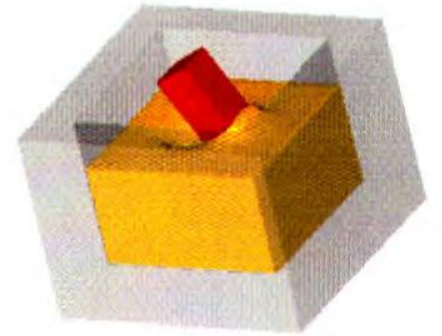

$t=0.001$

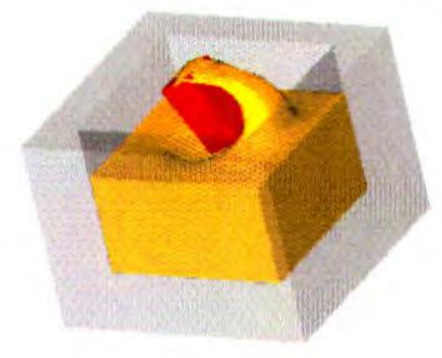

$t=0.0025$

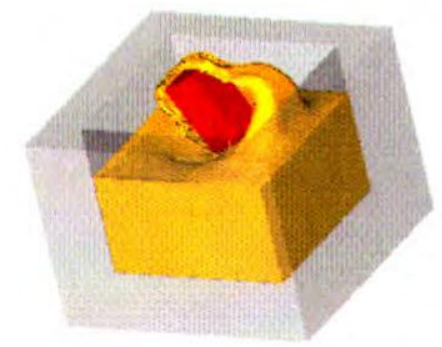

$$
t=0.004
$$

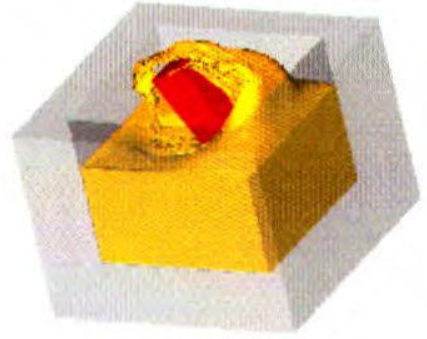

$t=0.0055$

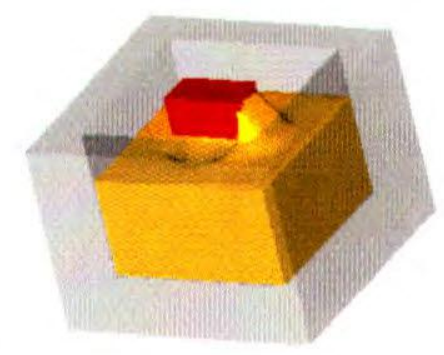

$t=0.0015$

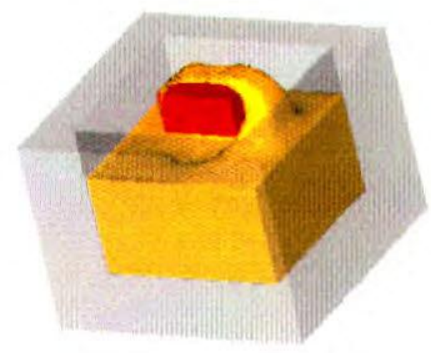

$$
t=0.003
$$

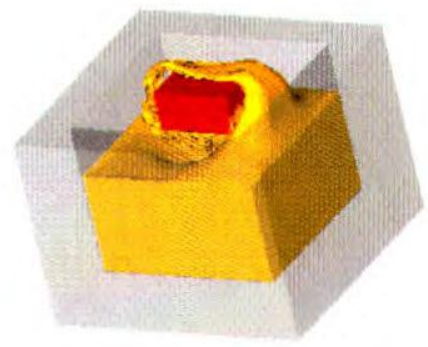

$$
t=0.0045
$$

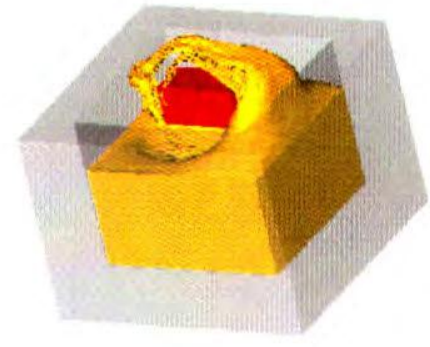

$$
t=0.006
$$




\section{Exemplo 2}

Este modelo de simulação possui um contêiner do tipo Box, contendo um fluido do tipo Cube, e um contêiner do tipo Cube, como mostra a figura 6.2. Os dados que configuram o escoamento são:

- Dimensões do domínio: $0.02 \times 0.02 \times 0.02$;

- Tamanho da malha: $20 \times 20 \times 20$ células;

- Tempo inicial: 0.0, tempo final: 0.006;

- Valor de referência para comprimento: 0.014;

- Valor de referência para velocidade: 1.0 ;

- Viscosidade: 0.0014;

- Número de Reynolds: 10.0;

- Número de Frouden: 2.698370;

- Número de Strouhal: 71.428571;

- Tipo de Movimento: Rotação.

- Tempo do ciclo de rotação : 0.006

O contêiner do tipo Cube, na cor azul, vai realizar o movimento de rotação no plano $x y$, encostando no fluido durante a simulação.

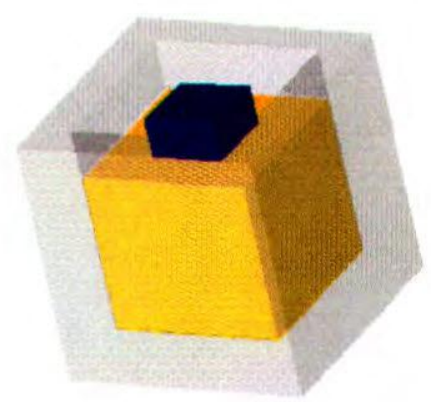

Figura 6.2: Objeto no instante $t=0.0$ 


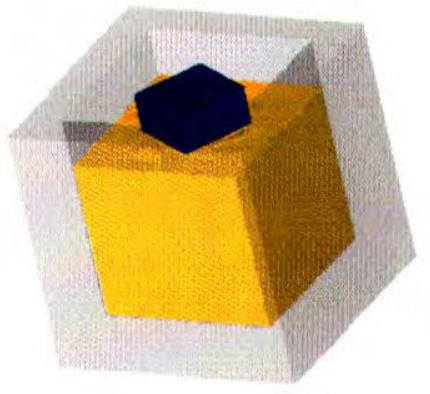

$t=0.001$

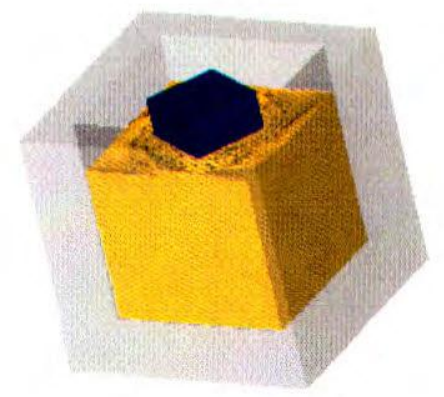

$t=0.004$

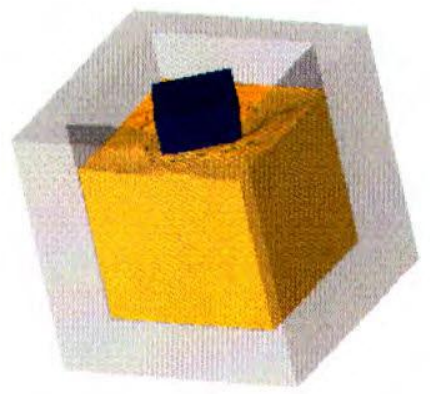

$t=0.002$

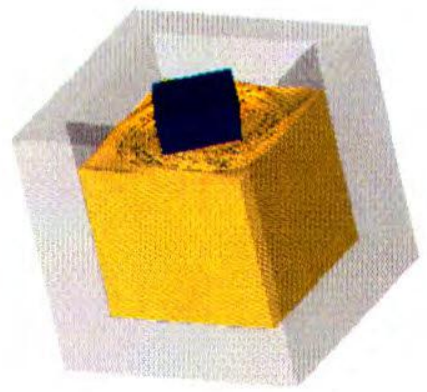

$$
t=0.005
$$

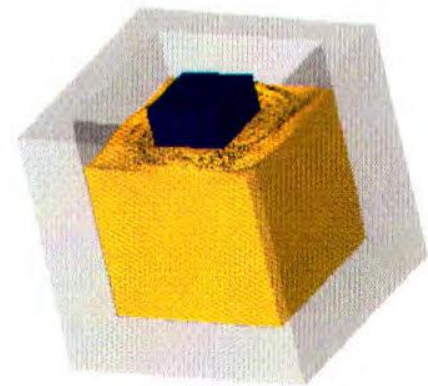

$$
t=0.006
$$

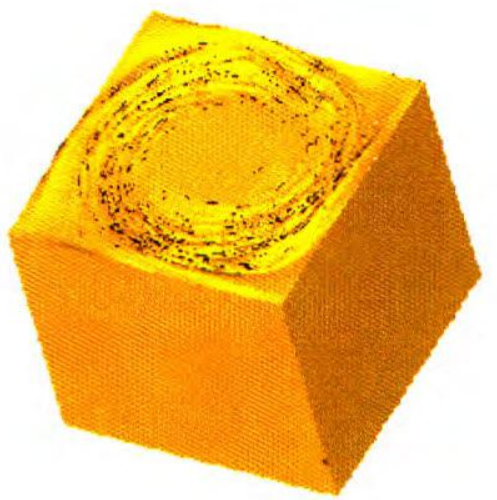

Figura 6.3: Fluido no instante $t=0.006$ 


\section{Exemplo 3}

Este modelo de simulaçāo possui um injetor do tipo Cylinder e um contêiner do tipo Cylindrical Cup. Os dados que configuram o escoamento são:

- Dimensões do domínio: 0.05×0.05×0.05;

- Tamanho da malha: $50 \times 50 \times 50$ células;

- Tempo inicial: 0.0, tempo final: 0.5;

- Valor de referência para comprimento: 0.006;

- Valor de referência para velocidade: 1.0 ;

- Viscosidade: 0.0006;

- Número de Reynolds: 10.0;

- Número de Frouden: 4.121828;

- Número de Strouhal: 166.666667;

- Tipo de Movimento: Harmônico simples

- Coordenada $x_{c}$ do Centro de Massa Inicial: 0.0250

- Coordenada $y_{c}$ do Centro de Massa Inicial: 0.0250

- Coordenada $z_{c}$ do Centro de Massa Inicial: 0.04650

- Coordenada $x_{f}$ do Centro de Massa Final: 0.0250

- Coordenada $y_{f}$ do Centro de Massa Final: 0.0250

- Coordenada $z_{f}$ do Centro de Massa Final: 0.03450

- Tempo do ciclo: 0.250

O injetor desloca-se ao longo do eixo $z$, aproximando-se e afastando-se do contêiner enquanto injeta o fluido. 


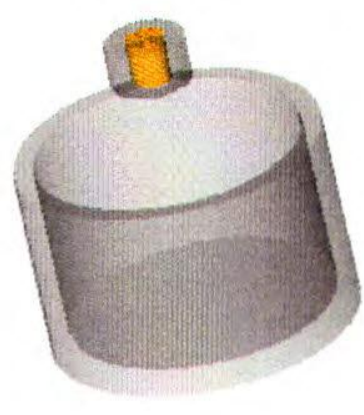

$$
t=0.0
$$

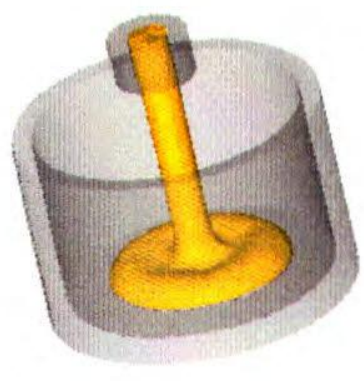

$$
t=0.1
$$

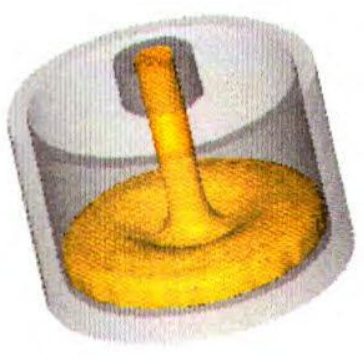

$$
t=0.25
$$

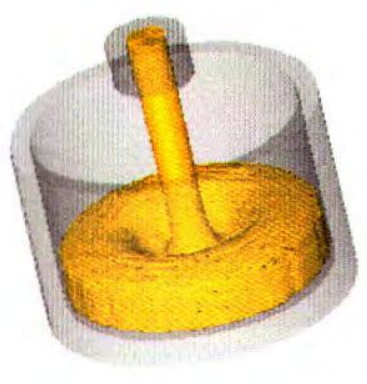

$$
t=0.4
$$

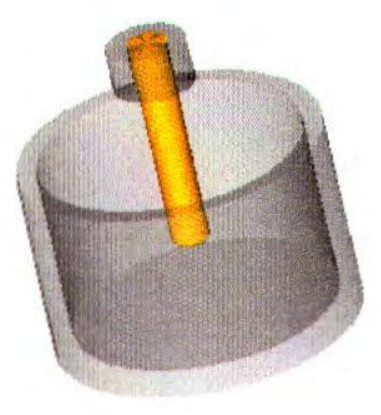

$$
t=0.025
$$

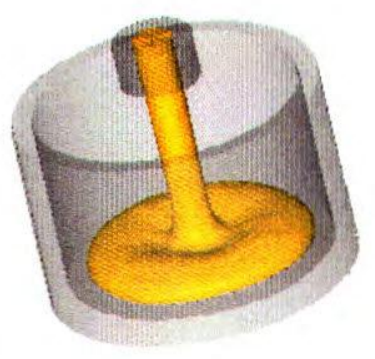

$$
t=0.15
$$

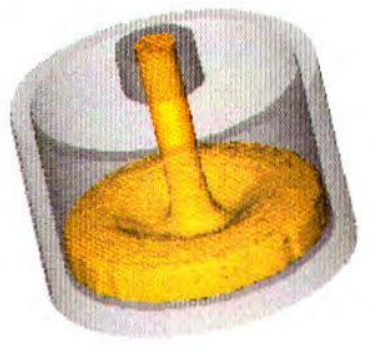

$$
t=0.3
$$

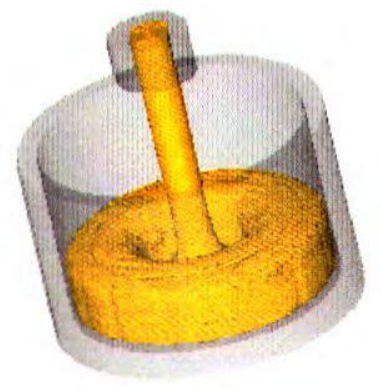

$t=0.45$

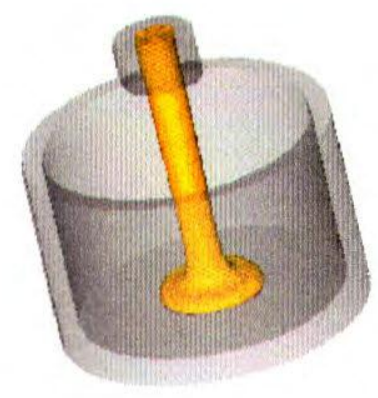

$$
t=0.05
$$

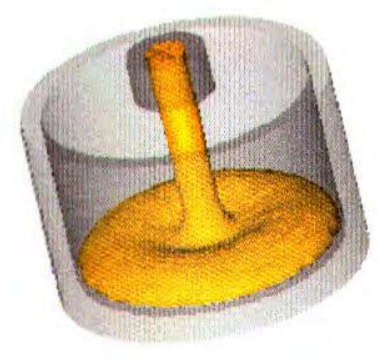

$$
t=0.2
$$

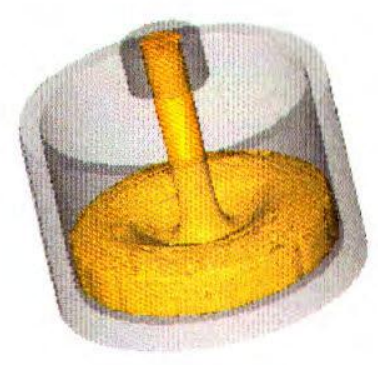

$$
t=0.35
$$

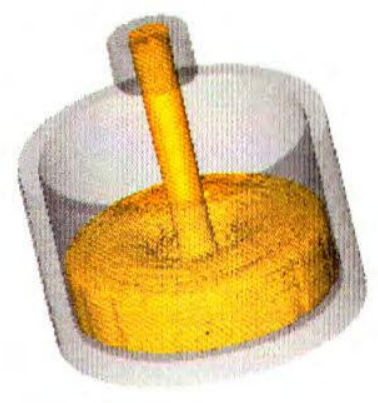

$$
t=0.5
$$




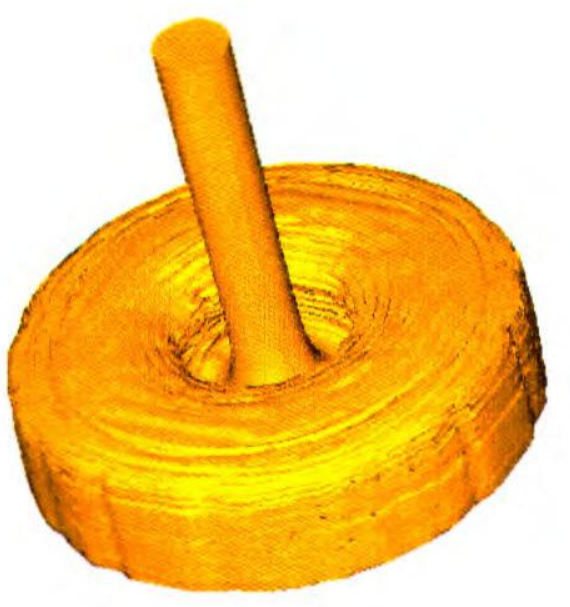

Figura 6.4: Fluido no instante $t=0.5$
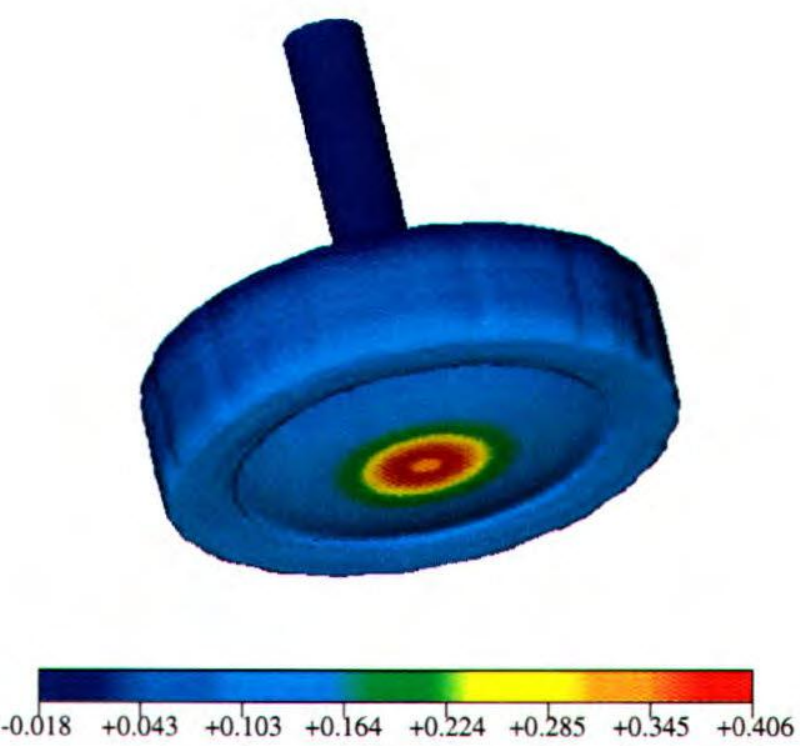

Figura 6.5: Pressão no instante $t=0.5$ 


\section{Exemplo 4}

Este modelo de simulação possui um injetor do tipo Cube, um contêiner do tipo Open Box e um contêiner do tipo Cube. Os dados que configuram o escoamento são:

- Dimensões do domínio: $0.02 \times 0.02 \times 0.02$;

- Tamanho da malha: $20 \times 20 \times 20$ células;

- Tempo inicial: 0.0, tempo final: 0.14;

- Valor de referência para comprimento: 0.004 ;

- Valor de referência para velocidade: 1.0 ;

- Viscosidade: 0.0004;

- Número de Reynolds: 10.0 ;

- Número de Frouden: 5.048188;

- Número de Strouhal: 250.0;

- Tipo de Movimento do injetor: Harmônico simples

- Coordenada $x_{c}$ do Centro de Massa Inicial: 0.010

- Coordenada $y_{c}$ do Centro de Massa Inicial: 0.010

- Coordenada $z_{c}$ do Centro de Massa Inicial: 0.01850

- Coordenada $x_{f}$ do Centro de Massa Final: 0.010

- Coordenada $y_{f}$ do Centro de Massa Final: 0.010

- Coordenada $z_{f}$ do Centro de Massa Final: 0.01550

- Tempo do ciclo: 0.060

- Tempo de parada do ciclo: 0.120

- Tipo de Movimento do contêiner tipo Cube: Rotação

- Tempo do ciclo: 0.0250

- Tempo de início do ciclo: 0.10

- Tempo de parada do ciclo: 0.140

No decorrer desta simulação, os objetos realizam movimentos independentes. $O$ injetor desloca-se para baixo enquanto injeta o fluido e a partir do instante $t=0.06$, para de injetar fluido e o movimento é revertido, retornando à posição inicial. No instante $t=$ 0.10 , o contêiner, na cor azul, realiza o movimento de rotação, misturando o fluido que encontrava-se sobre os contêineres. 


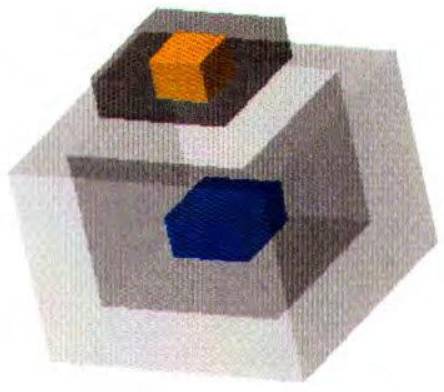

$$
t=0.0
$$

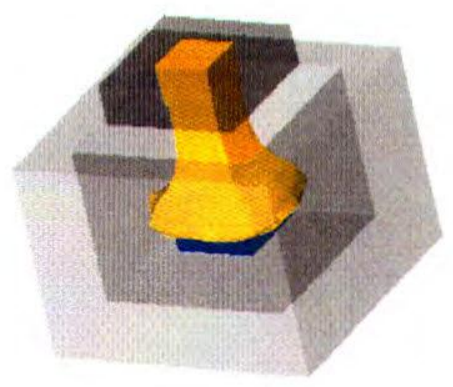

$$
t=0.02
$$

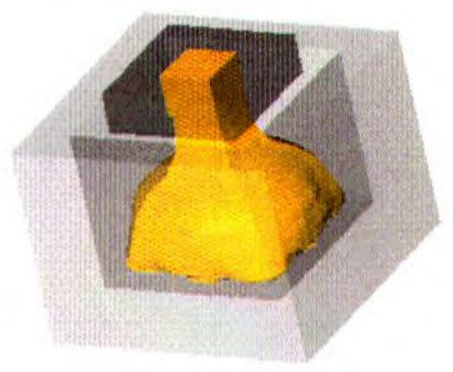

$$
t=0.05
$$

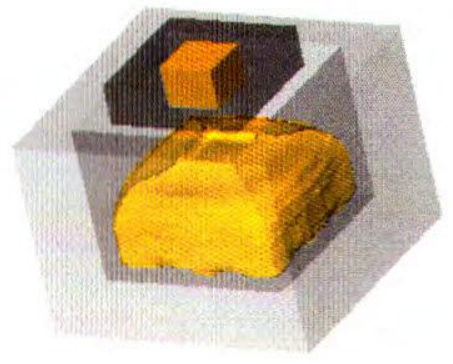

$$
t=0.075
$$

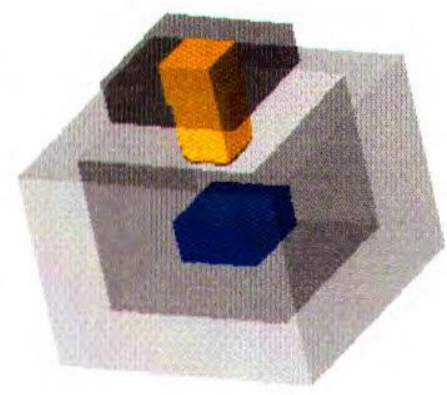

$$
t=0.005
$$

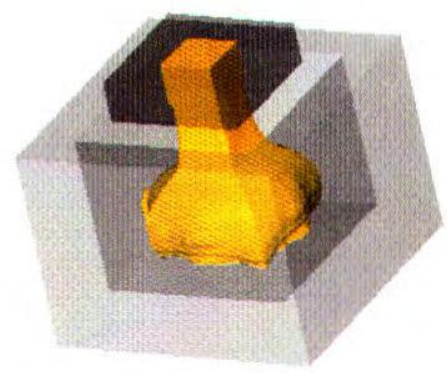

$$
t=0.03
$$

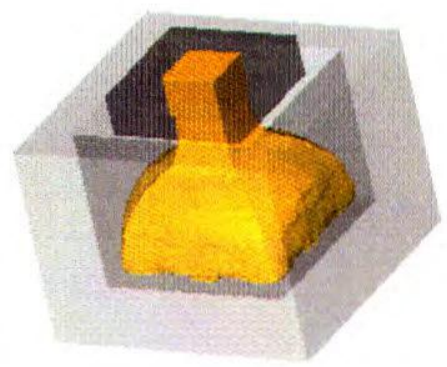

$t=0.06$

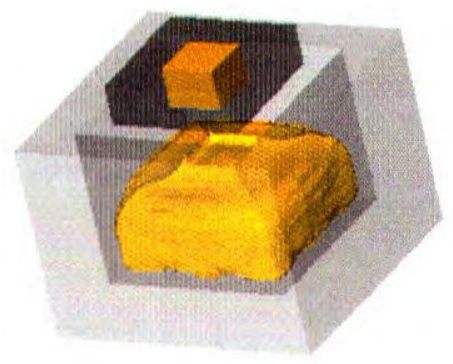

$t=0.08$

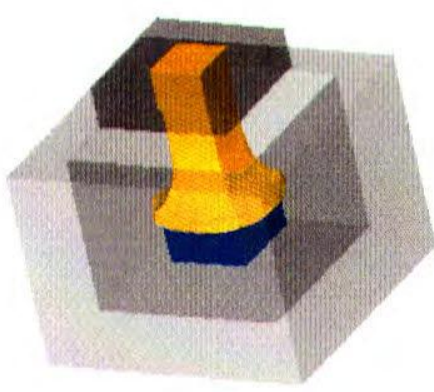

$$
t=0.015
$$

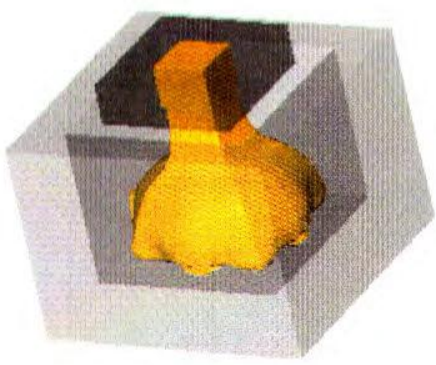

$$
t=0.04
$$

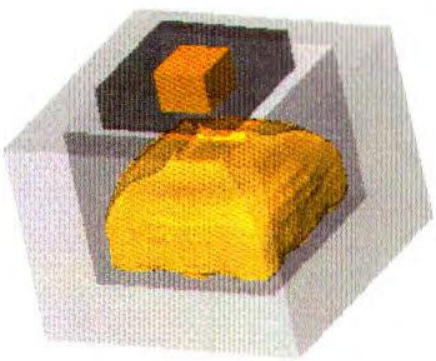

$$
t=0.07
$$

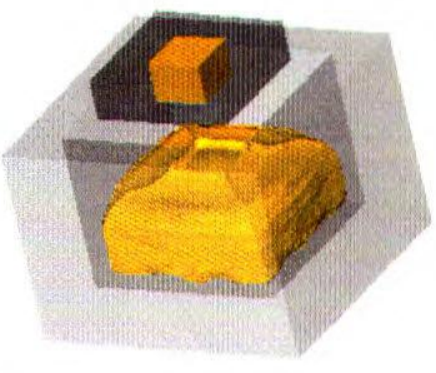

$t=0.09$ 


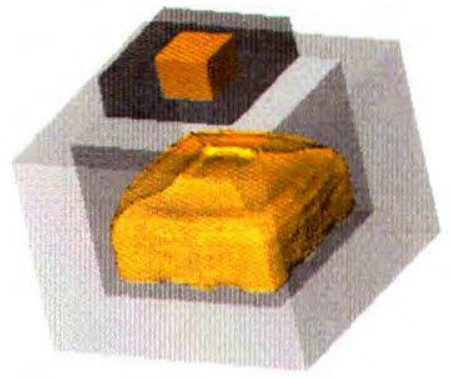

$t=0.1$

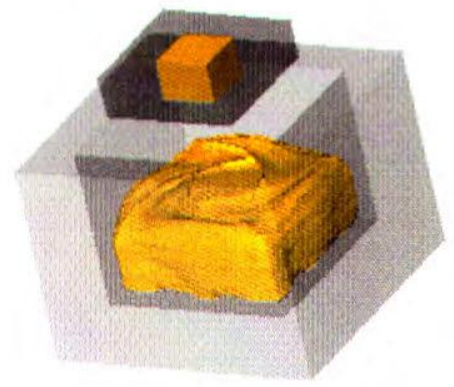

$t=0.115$

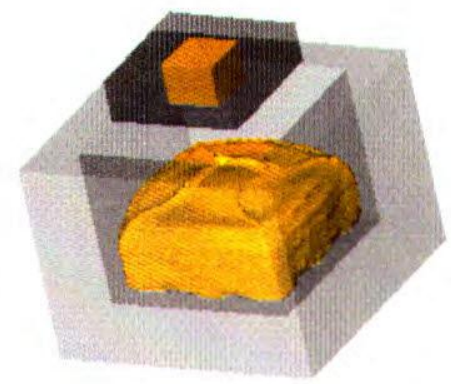

$t=0.105$

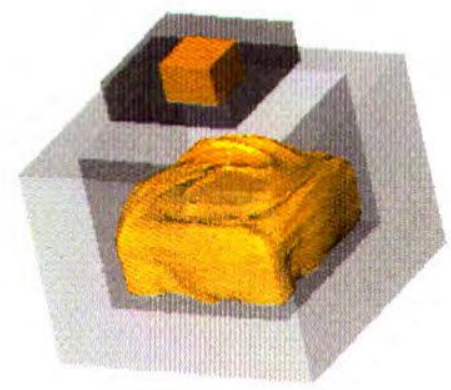

$t=0.12$

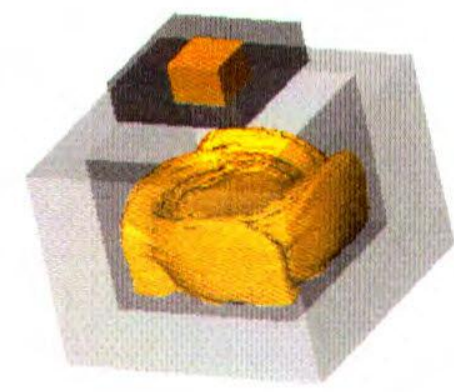

$t=0.14$

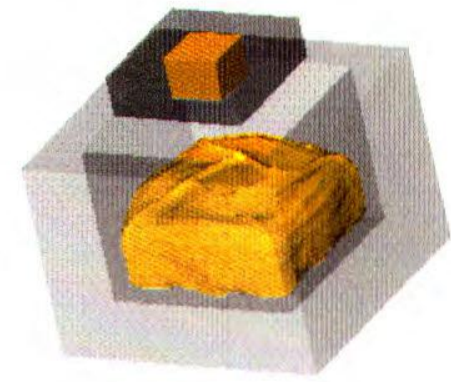

$t=0.11$$$
t=0.14
$$

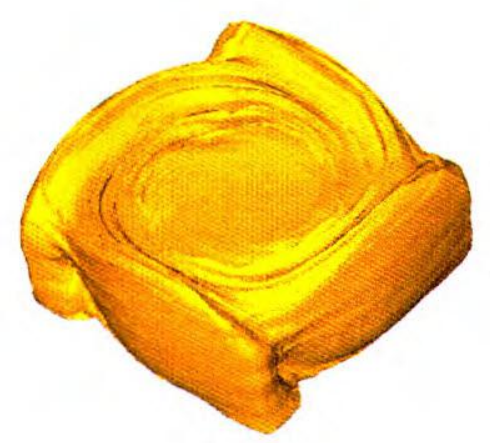

Figura 6.6: Fluido no instante $t=0.14$ 

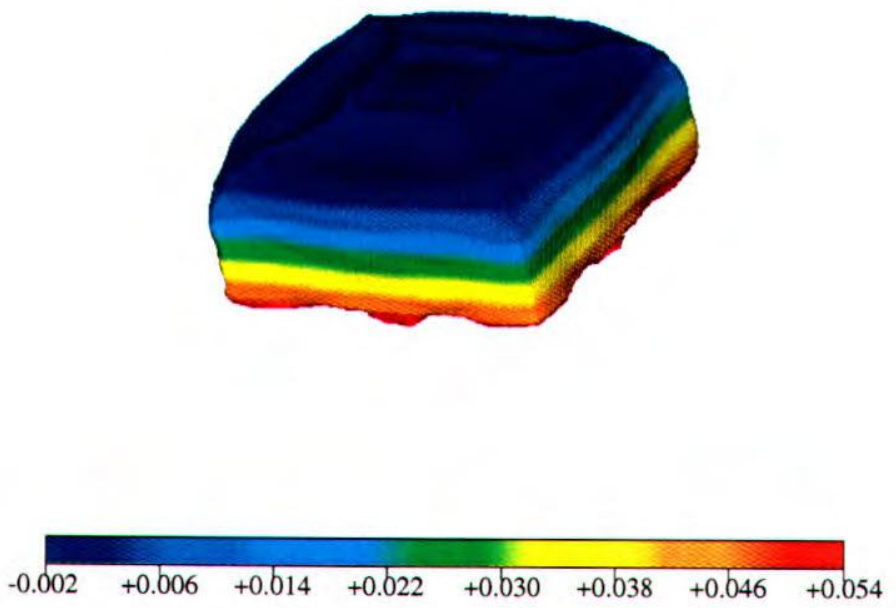

Figura 6.7: Pressão no instante $t=0.10$
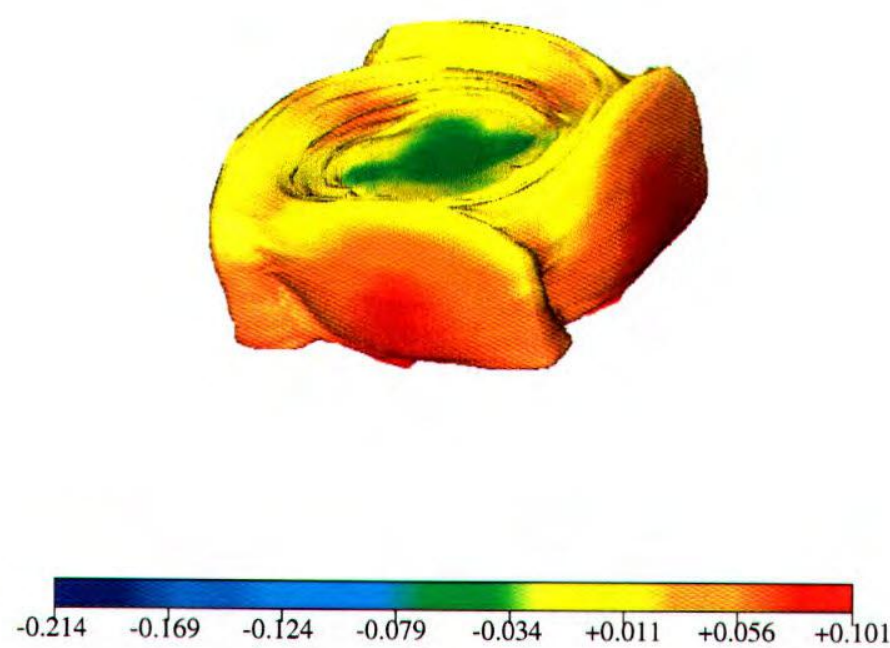

Figura 6.8: Pressão no instante $t=0.14$ 


\section{Exemplo 5}

Este modelo de simulaçāo possui um contêiner do tipo Box, contendo um fluido do tipo Cube, e um contêiner do tipo Cube. Os dados que configuram o escoamento são:

- Dimensões do domínio: $0.02 \times 0.02 \times 0.02$;

- Tamanho da malha: $20 \times 20 \times 20$ células;

- Tempo inicial: 0.0, tempo final: 0.0480;

- Valor de referência para comprimento: 0.014;

- Valor de referência para velocidade: 0.5 ;

- Viscosidade: 0.000350;

- Número de Reynolds: 20.0;

- Número de Frouden: 1.349185;

- Número de Strouhal: 35.714286;

- Tipo de Movimento: Harmônico simples

- Coordenada $x_{c}$ do Centro de Massa Inicial: 0.010

- Coordenada $y_{c}$ do Centro de Massa Inicial: 0.010

- Coordenada $z_{c}$ do Centro de Massa Inicial: 0.0180

- Coordenada $x_{f}$ do Centro de Massa Final: 0.010

- Coordenada $y_{f}$ do Centro de Massa Final: 0.010

- Coordenada $z_{f}$ do Centro de Massa Final: 0.0060

- Tempo do ciclo: 0.0060

- Tempo de parada do ciclo: 0.0060

Durante esta simulação o contêiner do tipo Cube vai realizar o movimento harmônico simples, mergulhando no fluido até atingir $\mathrm{t}=0.006$. Em seguida, o objeto permanece em posição constante até o final da simulação e o fluido cobre quase todo objeto. 


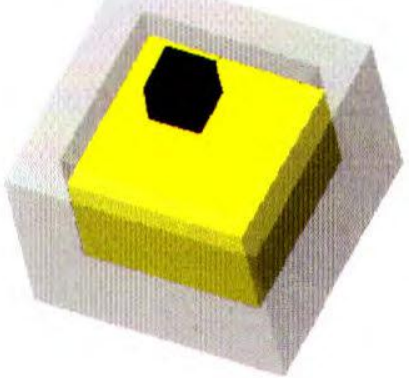

$t=0.0$

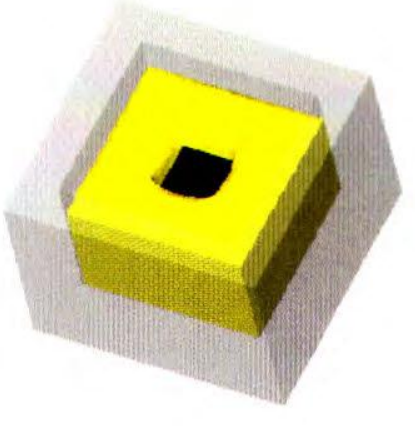

$t=0.004$

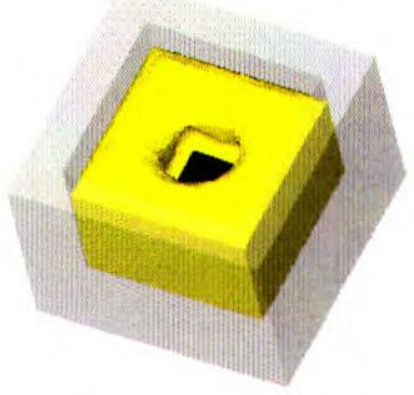

$t=0.009$

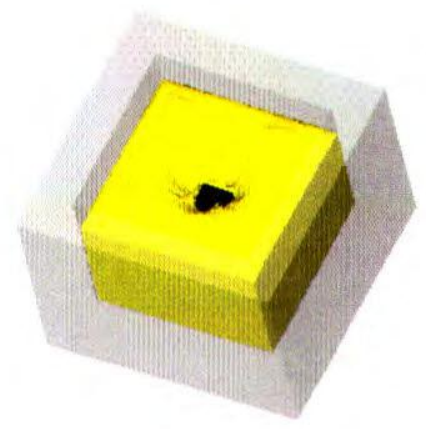

$t=0.03$

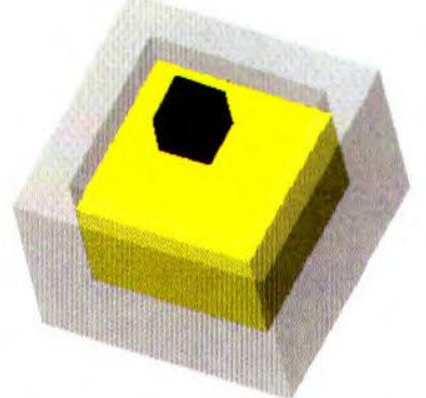

$t=0.001$

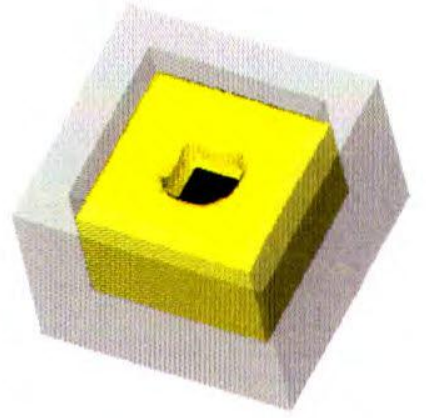

$t=0.005$

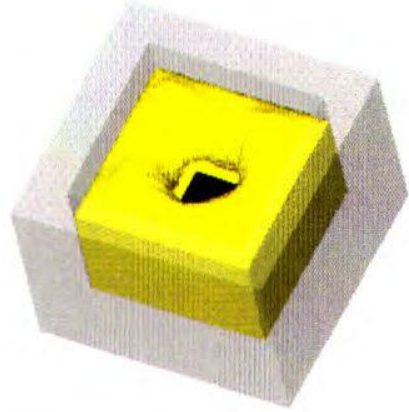

$t=0.018$

$$
t=0.024
$$

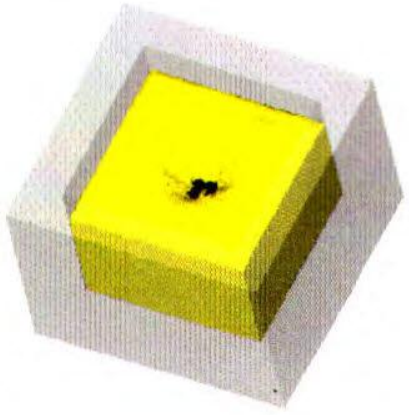

$t=0.039$

$t=0.048$ 


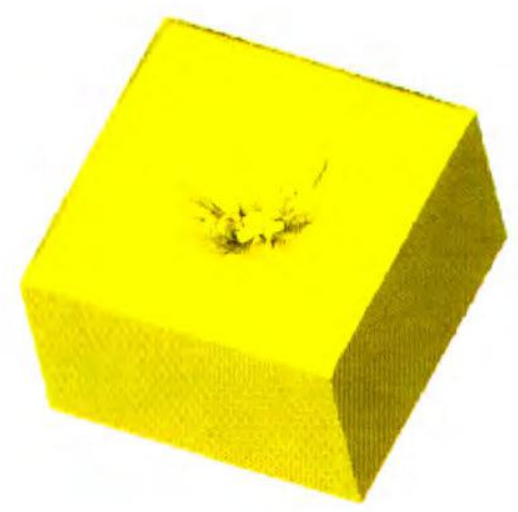

Figura 6.9: Fluido no instante $t=0.048$

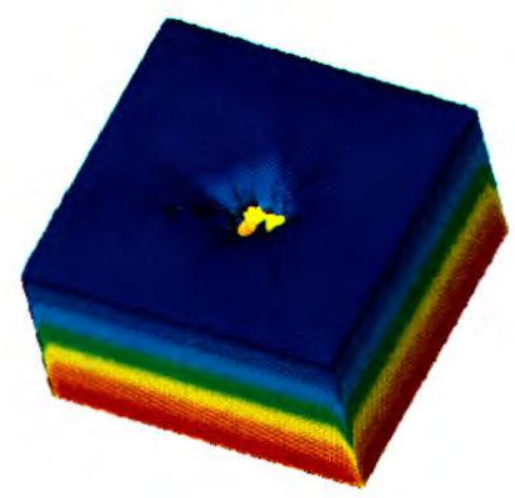

$-0.002+0.048+0.099+0.150+0.200+0.251+0.302+0.352$

Figura 6.10: Pressão no instante $t=0.048$ 


\section{Capítulo 7}

\section{Conclusões}

Este projeto de mestrado teve como objetivo o desenvolvimento de um modelador de movimentos para o sistema Freeflow-3D.

Este trabalho dividiu-se basicamente em duas etapas.

A primeira foi a realização de um levantamento bibliográfico sobre dinâmica de fluidos computacional, detalhado nos capítulos que compõem esta dissertação. No primeiro capítulo foram apresentados alguns conceitos fundamentais e o desenvolvimento das equações de Navier-Stokes, que regem os escoamentos transientes, laminares e incompressíveis. Essas equações são empregadas no método GENSMAC, descrito no capítulo 2, o qual tem como objetivo investigar a dinâmica de um fluido incompressível. Para isso, o método consiste numa técnica de diferenças finitas empregando variáveis primitivas de velocidade e pressão. O fluido é representado através de partículas virtuais cujas coordenadas são armazenadas e atualizadas no tempo e utiliza-se um procedimento para definir as células que identificam a região contendo o fluido e a superfície livre.

As discretizações e aproximações das equações de momento, da continuidade, de Poisson e das condiçōes de contorno foram detalhadas no capítulo 3, utilizando-se o método de diferenças finitas. Para a resolução da equação de Poisson, adota-se o método dos gradientes conjugados. Um procedimento de controle do passo no tempo é empregado para otimizar o tamanho do passo dado no tempo em cada ciclo de cálculo.

O capítulo 4 apresentou o sistema Freeflow-3D, um ambiente completo de simulação de escoamentos incompressíveis tridimensionais com superfícies livres. Este sistema é composto por um modelador - módulo Modflow-3D, que facilita a entrada de dados, um simulador - módulo Simflow-3D, que resolve as equaçōes governantes e as condições au- 
xiliares, um visualizador - módulo Visflow-3D, que permite a visualização dos resultados gerados pelo simulador, com imagens bi e tridimensionais do escoamento e um reiniciador - módulo Resimflow-3D, capaz de reiniciar uma simulação em qualquer fase. Este sistema emprega o método GENSMAC-3D que é uma extensão do método GENSMAC.

A próxima etapa passou a ser a implementação do modelador de movimentos. $\mathrm{O}$ capítulo 5 descreveu a metodologia utilizada para o seu desenvolvimento e a principal técnica empregada foi definir a trajetória dos objetos a partir do seu centro de massa. Três tipos de movimento foram implementados: rotação, harmônico simples e interpolação por splines cúbica, onde o usuário consegue criar um movimento para o objeto, de acordo com a inserção de alguns pontos de interpolação.

Inicialmente, desenvolveu-se o modelador de movimentos bidimensional, que está integrado ao sistema Freeflow-2D. Sua interface gráfica foi integrada ao módulo Modflow$2 \mathrm{D}$ e utiliza o sistema de gerenciamento de janelas Xview sobre o Xwindows.

Após o funcionamento do modelador de movimentos bidimensional, passou-se então ao desenvolvimento de sua extensão tridimensional, realizando-se algumas adaptações.

O modelador de movimentos permite que os objetos realizem movimentos independentes durante a simulação e o usuário tem a disponibilidade de escolher os parâmetros relacionados a cada tipo de movimento.

O capítulo 6 apresentou alguns exemplos de simulaçōes com objetos em movimento. Tratou-se de exemplos com aplicação prática principalmente no preenchimento de moldes e cavidades utilizados por vários de tipos de indústrias. Durante o processo de enchimento, as indústrias têm grande interesse em estudar o comportamento do escoamento para evitar falhas, controlar o tempo de fabricação e não prejudicar a qualidade de seus produtos.

Este trabalho contribuiu para a extensão do sistema Freeflow-3D, permitindo simulações com objetos em movimento.

Até o momento, não foi encontrada na literatura nenhum resultado de simulação relacionado ao movimento de contêineres e injetores, o que impede a comparação com os resultados obtidos pelo modelador de movimentos. Acredita-se que este apresentou resultados compatíveis com os escoamentos observados, tornando-se assim, uma ferramenta muito útil para as indústrias. 
O modelador de movimentos possui uma estrutura que permite sua extensão para outros tipos de movimento, por exemplo, movimento definido por uma aproximação linear por partes. Outra proposta para futura extensão deste trabalho são algumas modificações relacionadas a implementação de-leis da física, responsáveis pela interação das forças que envolvem o movimento do fluido com o movimento do objeto.

Uma interessante proposta de extensão do sistema Freeflow-3D seria a implementação de um método de animaçāo no visualizador, módulo Visflow-3D, para melhor visualizar as simulações realizadas com o modelador de movimentos. 


\section{Referências Bibliográficas}

[1] ACHESON, D.J. Elementary Fluid Dynamics. Oxford University Press, Nova York, 1992.

[2] AMSDEN, A.; HARLOW, F. The SMAC Method: A Numerical Technique for Calculating Incompressible Fluid Flows. Los Alamos Scientific Laboratory Report LA-4370, Los Alamos, NM, 1970.

[3] CASTELO FILHO, A.; TOMÉ, M. F.; CÉSAR, C. N. L.; MCKEE, S., CUMINATO, J. A. Freeflow: An Integrated Simulation System for Three-Dimensional Free Surface Flows. Computing and Visualization in Science, V.2, pp. 1-12, 1999.

[4] CASTELO FILHO, A., LOPES, H. C. V.; TAVARES S., G. Topological Methods in Geometric Modeling. In: Congresso Nacional de Matemática Aplicada e Computacional - CNMAC, 15.,1992. [São Carlos, SP: SBMAC, 1992.]

[5] FERREIRA, V. G.; AZEVEDO, J. L. F.; CUMINATO, J. A. Métodos Numéricos para Escoamento Incompressivel. Notas Didáticas do ICMC-USP, nํ2 26, 1997.

[6] FERREIRA, V. G.; MELLO, O. D.; OLIVEIRA, J. N.; FORTUNA, A. O. Tópicos Teóricos e Computacionais em Escoamentos de Fluidos. Notas do ICMC-USP, $n^{\circ} \mathbf{3 9}$, 1998.

[7] FLETCHER, C. A. J. Computational Techniques for Fluid Dynamics. SpringerVerlag, New York, Vol. I and Vol. II, 1988.

[8] FORTUNA, A. O. Técnicas Computacionais para Dinâmica dos Fluidos. Editora da Universidade de São Paulo (EDUSP), 2000.

[9] FOX, R. W.; MCDONALD A. T. Introdução à Mecânica dos Fluidos. Livros Técnicos e Científicos, 1995. 
[10] GRIEBEL, M.; DORNSEIFER, T.; NEUNHOEFFER, T. Numerical Simulation in Fluid Dynamics. Society for Industrial and Applied Mathematics (SIAM) Press, Philadelphia, 1998.

[11] GROSSI, L. Solução Numérica para Escoamentos Não-Newtonianos com Superfícies Livres. Exame de Qualificação, ICMC-USP, 1996.

[12] HARLOW, F. H.; WELCH, J. E. Welch (1965), The MAC Method. Phys. Fluids, V.8, 2182; WELCH, J. E.; HARLOW, F. H.; SHANNON, J. P.; DALY, B. J. Los Alamos Scientific Laboratory, Report LA-3425, Los Alamos, NM, 1996.

[13] HIRT, C. W.; NICHOLS, B. D. Volume of Fluid (VOF) Method for the Dynamics of Free Boundaries. J. Comp. Phys., V.39, 201, 1981.

[14] MALISKA, C.R. Transferência de Calor e Mecânica dos Fluidos Computacional. LTC Editora, Rio de Janeiro, 1995.

[15] NOBLE, B. Álgebra Linear Aplicada. Prentice-Hall, 1986.

[16] OLIVEIRA, J. Desenvolvimento de um Sistema de Simulação de Escoamentos de Fluidos com Superfícies Livres Bidimensionais. Dissertação de Mestrado, ICMC-USP, 1999.

[17] PEYRET, R.; TAYLOR, T. D. Computational Methods for Fluid Flow. SpringerVerlag, New York, 1983.

[18] RUGGIERO, M. A. G., LOPES, V. L. R. Cáculo Numérico 2 edição, Makron Books, São Paulo, 1997.

[19] SANTOS, L. C. C. Introdução à Mecânica dos Fluidos Computacional. In: Congresso Nacional de Matemática Aplicada e Computacional - CNMAC, 21.,1998. [Caxambu, MG: SBMAC, 1998.] 76 p.

[20] ȘCHILDT, H. C Completo e Total. Makron Books, 1996.

[21] SMITH, G. D. Numerical Solution of Partial Differential Equations: Finite Difference Methods. Oxford University Press, Oxford, 1978. 
[22] TOMÉ, M. F.; MCKEE, S. GENSMAC: A Computational Marker-and-Cell Method for Free Surface Flows in General Domains. J. Comp. Phys., V.110, n.1, pp.171-186, 1994.

[23] TOMÉ, M. F.; DUFFY, B.; MCKEE, S. A Numerical Method for Solving Unsteady Non-Newtonian Free Surface Flows. Journal of Non-Newtonian Fluid Mechanics, V. 62, pp.9-34,1996.

[24] TOMÉ, M. F.; CASTELO F., A.; CUMINATO, J. A.; MCKEE, S. GENSMAC-3D: Inplementation of the Navier-Stokes Equations and Boundary Conditions for $3 D$ Free Surface Flows. Notas do ICMC-USP, $n^{\circ}$ 29, (1996).

[25] YOUNG, D. A.; PEW, J. A. The XWindow System - Programming and Applications with Xt. Prentice-Hall International, Inc, 1992. 